\title{
Amide-Based Cinchona Alkaloids as Phase-Transfer Catalysts: \\ Synthesis and Potential Application
}

Maciej Majdecki, Patryk Niedbala, Janusz Jurczak*

Institute of Organic Chemistry, Polish Academy of Sciences, Kasprzaka 44/52, 01-224 Warsaw, Poland Contact e-mail: jurczak group@icho.edu.pl

\section{Contents}

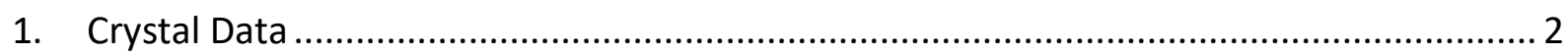

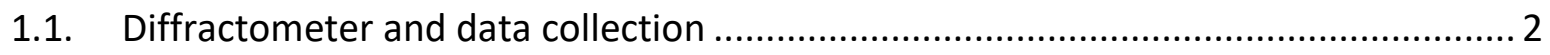

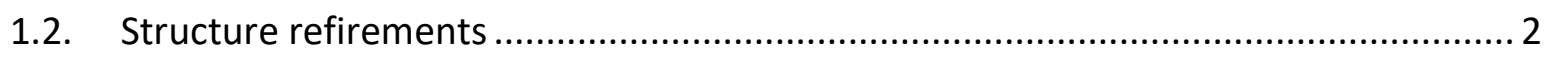

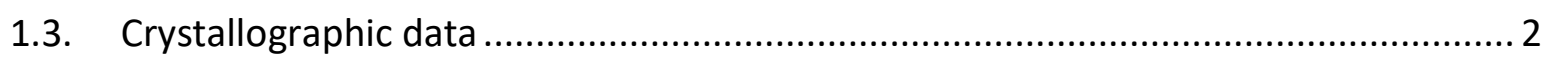

1.4. Crystal data and structure refinement details ............................................... 2

1.5. Thermal ellipsoid plots (ORTEP) for reported crystal structure ............................. 3

2. DFT studies and corresponding Cartesian coordinates .............................................. 4

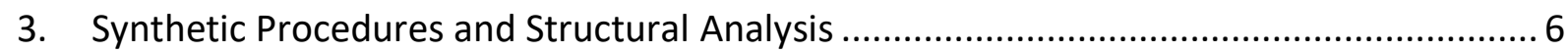

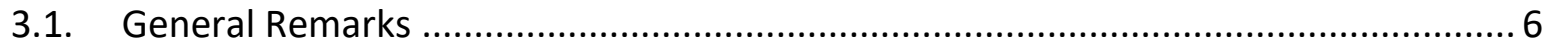

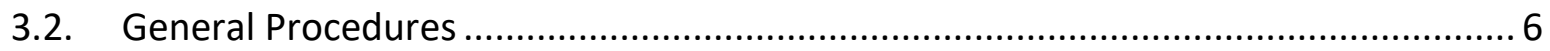

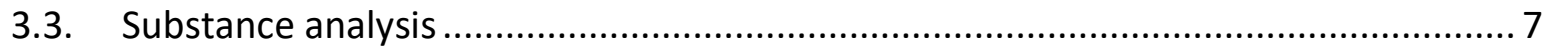

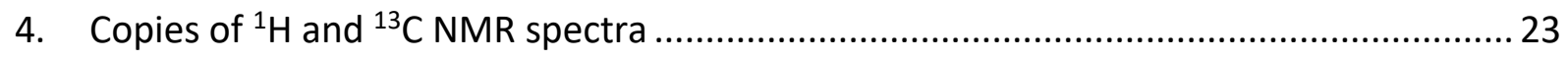

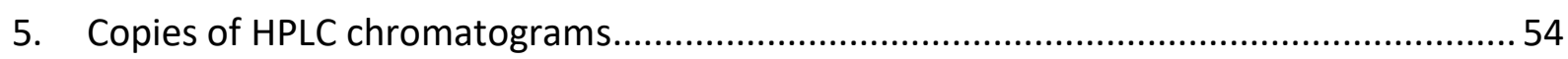

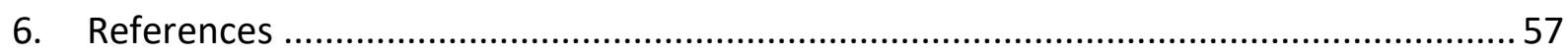




\section{Crystal Data}

\subsection{Diffractometer and data collection}

Single crystal X-ray diffraction measurements were carried out on a Agilent Supernova diffractometer, at $100 \mathrm{~K}$ with graphite monochromated $\mathrm{Cu}$ Ka radiation (1.54184 $\AA$ ). Colorless crystal of approximate dimensions $0.6 \times 0.06 \times 0.03 \mathrm{~mm}$ (long needle) was used.

\subsection{Structure refirements}

The data reduction was made by using CrysAlisPRO ${ }^{1}$ software. The structures were solved by direct methods and refined on F2 by full-matrix least-squares by using SHELXS97 and SHELXL97. ${ }^{2}$ All non-hydrogen atoms were refined as anisotropic while hydrogen atoms were placed in calculated positions, and refined in riding mode.

\subsection{Crystallographic data}

CCDC contains the supplementary crystallographic data for this paper. These data can be obtained free of charge from The Cambridge Crystallographic Data Centre via www.ccdc.cam.ac.uk/data_request/cif

\subsection{Crystal data and structure refinement details}

Tab. S1 Crystal data and structure refinement details for $\mathbf{3 7 .}$

\begin{tabular}{|c|c|}
\hline \multirow{2}{*}{ Compound } & $\mid$ \\
\hline Empirical formula & $\mathrm{C}_{27} \mathrm{H}_{27} \mathrm{BrF}_{3} \mathrm{~N}_{3} \mathrm{O}_{2}$ \\
\hline Moiety formula & $\mathrm{C}_{27} \mathrm{H}_{27} \mathrm{~F}_{3} \mathrm{~N}_{3} \mathrm{O}_{2}{ }^{+}, \mathrm{Br}-$ \\
\hline Formula weight & 562.43 \\
\hline CCDC No. & 1949618 \\
\hline Wavelength & 1.54184 \\
\hline Crystal system & orthorhombic \\
\hline Space group & $P 2_{1} 2_{1} 2_{1}$ \\
\hline & $a=8.1994(2) \AA$ \\
\hline Unit cell dimensions & $b=10.6269(3) \AA$ \\
\hline & $c=27.3873(7) \AA$ \\
\hline Volume & $2386.37(11) \AA^{3}$ \\
\hline Z & 4 \\
\hline Density Calc. & $1.565 \mathrm{~g} / \mathrm{cm}^{3}$ \\
\hline Absorption coefficient & 2.827 \\
\hline F(000) & 1152 \\
\hline Crystal & $0.6 \times 0.06 \times 0.03 \mathrm{~mm}$ \\
\hline
\end{tabular}




\begin{tabular}{|c|c|}
\hline Index ranges & $-8 \leq \mathrm{h} \leq 9,-12 \leq \mathrm{k} \leq 12,-33 \leq 1 \leq 24$ \\
\hline $\begin{array}{c}\text { Reflections collected } \\
\text { (all / independent) }\end{array}$ & $7992 / 4193\left[R_{\text {int }}=0.0326\right]$ \\
\hline Absorption correction & multi-scan \\
\hline Refinement method & Full-matrix least-squares on $F^{2}$ \\
\hline Restraints / parameters & $0 / 406$ \\
\hline Goodness-of-fit on $F^{2}$ & 1.047 \\
\hline Final R indices $\left[F^{2}>2 \sigma\left(F^{2}\right)\right]$ & $R_{l}=0.0362, \omega \mathrm{R}_{2}=0.0934$ \\
\hline$R$ indices (all data) & $R_{l}=0.0398, \omega \mathrm{R}_{2}=0.0965$ \\
\hline
\end{tabular}

\subsection{Thermal ellipsoid plots (ORTEP) for reported crystal structure}

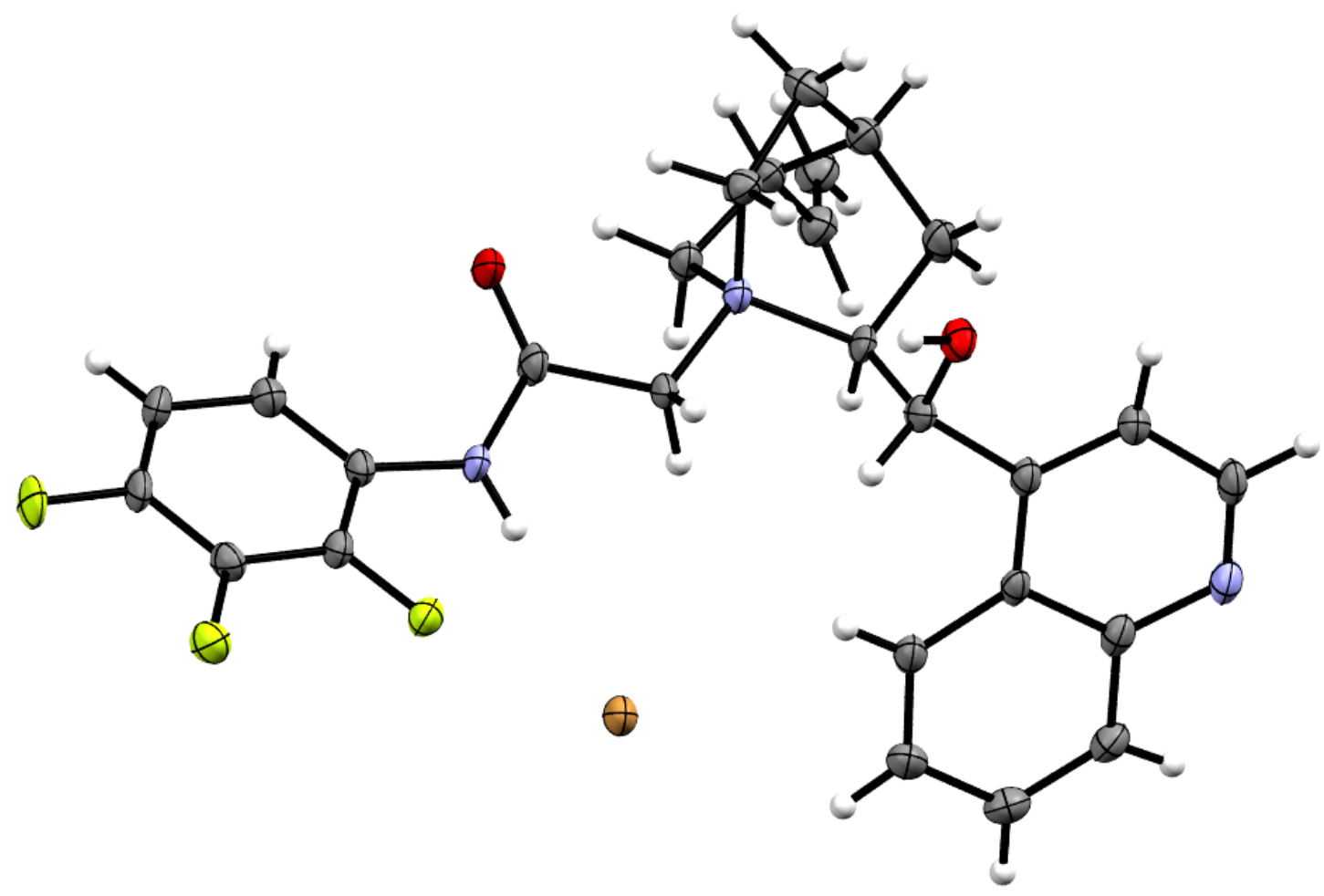

Fig. S1. Thermal ellipsoid plot (ORTEP) diagram for structure 37. 


\section{DFT studies and corresponding Cartesian coordinates}

a)

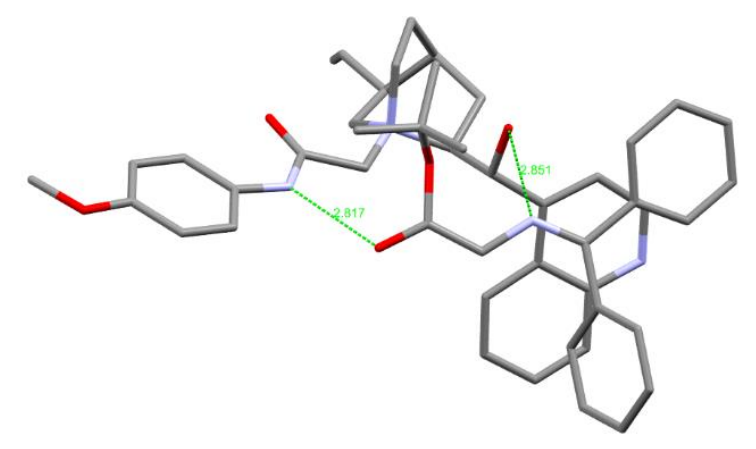

b)

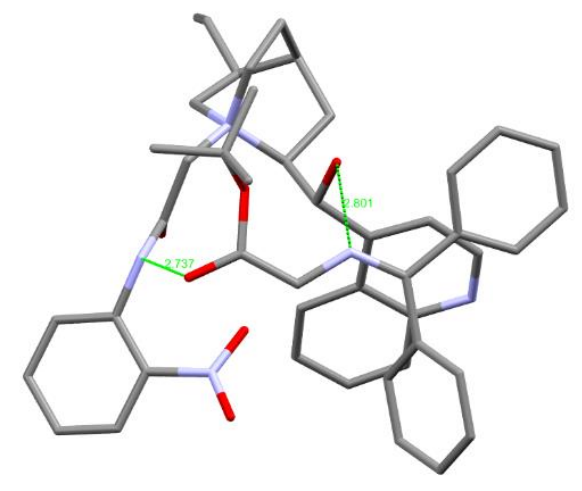

Fig. S2. Energy-minimized structures of complexes of catalysts $\mathbf{2 6}$ (a) and $\mathbf{3 1}$ (b) with substrate 48, obtained at DFT/M06-2X/6-31G(d) level of theory using program Spartan'16 Parallel Suite.

Tab. S2 Cartesian coordinates of the structures of catalysts $\mathbf{2 6}$ and $\mathbf{3 1}$ and substrate $\mathbf{4 8}$ complexes.

\begin{tabular}{|c|c|c|c|c|c|c|c|}
\hline \multicolumn{4}{|c|}{$\begin{array}{c}26 \text { compelx } \\
E=-2415.726258 \mathrm{au}\end{array}$} & \multicolumn{4}{|c|}{$\begin{array}{c}31 \text { compelx } \\
E=-2505.674630 \mathrm{au}\end{array}$} \\
\hline $\mathrm{C}$ & -6.191598 & -2.750273 & -1.315783 & $\mathrm{C}$ & 0.866678 & 5.533520 & 0.180441 \\
\hline $\mathrm{C}$ & -4.973254 & -2.129465 & -1.129609 & $\mathrm{C}$ & 0.931394 & 4.176096 & 0.475808 \\
\hline $\mathrm{C}$ & -4.913488 & -0.792310 & -0.713211 & $\mathrm{C}$ & 2.125531 & 3.581637 & 0.899691 \\
\hline $\mathrm{C}$ & -6.094586 & -0.092642 & -0.487578 & $\mathrm{C}$ & 3.264663 & 4.376428 & 0.972705 \\
\hline $\mathrm{C}$ & -7.323495 & -0.722168 & -0.679348 & $\mathrm{C}$ & 3.219350 & 5.723268 & 0.637514 \\
\hline $\mathrm{C}$ & -7.379758 & -2.051028 & -1.091018 & $\mathrm{C}$ & 2.014318 & 6.307824 & 0.256301 \\
\hline $\mathrm{N}$ & -3.618754 & -0.251619 & -0.553460 & $\mathrm{~N}$ & 2.185794 & 2.242354 & 1.350027 \\
\hline $\mathrm{C}$ & -3.282230 & 0.986182 & -0.153131 & $\mathrm{C}$ & 2.737200 & 1.279579 & 0.579851 \\
\hline $\mathrm{O}$ & -4.051467 & 1.876544 & 0.197765 & 0 & 3.258981 & 1.470499 & -0.512563 \\
\hline $\mathrm{C}$ & -1.759315 & 1.152014 & -0.206872 & $\mathrm{C}$ & 2.722230 & -0.081304 & 1.266160 \\
\hline $\mathrm{C}$ & 2.099391 & 1.104903 & -3.926614 & $\mathrm{C}$ & 0.673453 & 1.890169 & -3.787099 \\
\hline $\mathrm{C}$ & 3.436632 & 1.292335 & -4.331720 & $\mathrm{C}$ & -0.151088 & 1.706626 & -4.915305 \\
\hline $\mathrm{C}$ & 4.414459 & 1.514369 & -3.395892 & $\mathrm{C}$ & -1.012856 & 0.641317 & -4.964150 \\
\hline $\mathrm{C}$ & 4.104286 & 1.557595 & -2.016585 & $\mathrm{C}$ & -1.085081 & -0.284063 & -3.896643 \\
\hline $\mathrm{C}$ & 2.753413 & 1.369074 & -1.600049 & $\mathrm{C}$ & -0.247573 & -0.105836 & -2.753676 \\
\hline $\mathrm{C}$ & 1.766521 & 1.144239 & -2.594479 & $\mathrm{C}$ & 0.627518 & 1.009665 & -2.733917 \\
\hline $\mathrm{N}$ & 5.129269 & 1.768807 & -1.145710 & $\mathrm{~N}$ & -1.965727 & -1.314578 & -4.026335 \\
\hline $\mathrm{C}$ & 4.840314 & 1.757454 & 0.132859 & $\mathrm{C}$ & -2.039771 & -2.165603 & -3.033384 \\
\hline $\mathrm{C}$ & 3.546873 & 1.549184 & 0.660084 & $\mathrm{C}$ & -1.264535 & -2.088757 & -1.855797 \\
\hline $\mathrm{C}$ & 2.492947 & 1.366644 & -0.197881 & $\mathrm{C}$ & -0.363081 & -1.065506 & -1.701670 \\
\hline $\mathrm{C}$ & 1.089014 & 1.139674 & 0.328871 & $\mathrm{C}$ & 0.479413 & -1.000743 & -0.431498 \\
\hline $\mathrm{C}$ & 0.326679 & 2.462815 & 0.237709 & $\mathrm{C}$ & 1.925872 & -1.349959 & -0.807506 \\
\hline $\mathrm{O}$ & 1.067404 & 0.669227 & 1.642550 & $\mathrm{O}$ & 0.046736 & -1.903730 & 0.544301 \\
\hline $\mathrm{N}$ & -1.204127 & 2.368018 & 0.459297 & $\mathrm{~N}$ & 2.929436 & -1.263970 & 0.370104 \\
\hline $\mathrm{C}$ & -1.793994 & 3.637893 & -0.122255 & $\mathrm{C}$ & 4.330671 & -1.248815 & -0.211400 \\
\hline $\mathrm{C}$ & -1.040983 & 4.873574 & 0.391366 & $\mathrm{C}$ & 4.495115 & -2.363582 & -1.253890 \\
\hline $\mathrm{C}$ & -0.261076 & 4.446226 & 1.646113 & $\mathrm{C}$ & 3.376022 & -3.390314 & -1.012870 \\
\hline $\mathrm{C}$ & 0.868017 & 3.522915 & 1.200891 & $\mathrm{C}$ & 2.050731 & -2.753424 & -1.415353 \\
\hline $\mathrm{C}$ & -1.168732 & 3.657939 & 2.588027 & $\mathrm{C}$ & 3.296000 & -3.735784 & 0.471882 \\
\hline $\mathrm{C}$ & -1.508209 & 2.310579 & 1.946626 & $\mathrm{C}$ & 2.819152 & -2.503836 & 1.243662 \\
\hline $\mathrm{C}$ & -1.988378 & 6.008186 & 0.624981 & $\mathrm{C}$ & 5.875163 & -2.940735 & -1.195998 \\
\hline $\mathrm{C}$ & -1.892185 & 7.192344 & 0.028938 & $\mathrm{C}$ & 6.735041 & -2.925450 & -2.208768 \\
\hline $\mathrm{H}$ & -6.252277 & -3.787539 & -1.637428 & $\mathrm{H}$ & -0.088909 & 5.950614 & -0.124525 \\
\hline $\mathrm{H}$ & -6.057230 & 0.940124 & -0.160218 & $\mathrm{H}$ & 4.187698 & 3.914021 & 1.316797 \\
\hline $\mathrm{H}$ & -8.233125 & -0.155526 & -0.497900 & $\mathrm{H}$ & 4.125002 & 6.323761 & 0.692538 \\
\hline
\end{tabular}




\begin{tabular}{|c|c|c|c|c|c|c|c|}
\hline $\mathrm{H}$ & -1.318501 & 0.286330 & 0.300194 & $\mathrm{H}$ & 1.968870 & 7.366197 & 0.010376 \\
\hline $\mathrm{H}$ & -1.426953 & 1.163736 & -1.257355 & $\mathrm{H}$ & 1.477075 & 2.012282 & 2.083851 \\
\hline $\mathrm{H}$ & 1.328319 & 0.915241 & -4.671303 & $\mathrm{H}$ & 3.550263 & -0.101965 & 1.989895 \\
\hline $\mathrm{H}$ & 3.690297 & 1.256469 & -5.389974 & $\mathrm{H}$ & 1.790440 & -0.243025 & 1.830434 \\
\hline $\mathrm{H}$ & 5.458473 & 1.656162 & -3.670184 & $\mathrm{H}$ & 1.348980 & 2.742500 & -3.740643 \\
\hline $\mathrm{H}$ & 0.733104 & 0.951615 & -2.305093 & $\mathrm{H}$ & -0.104977 & 2.413901 & -5.741587 \\
\hline $\mathrm{H}$ & 5.672034 & 1.911286 & 0.824646 & $\mathrm{H}$ & -1.671412 & 0.464501 & -5.812073 \\
\hline $\mathrm{H}$ & 3.395384 & 1.492690 & 1.736204 & $\mathrm{H}$ & 1.255021 & 1.207834 & -1.868587 \\
\hline $\mathrm{H}$ & 0.624421 & 0.394738 & -0.335818 & $\mathrm{H}$ & -2.753629 & -2.985762 & -3.142274 \\
\hline $\mathrm{H}$ & 0.401319 & 2.812160 & -0.803190 & $\mathrm{H}$ & -1.398498 & -2.826661 & -1.067663 \\
\hline $\mathrm{H}$ & 1.163667 & -0.316616 & 1.557163 & $\mathrm{H}$ & 0.429697 & 0.035039 & -0.052371 \\
\hline $\mathrm{H}$ & -1.742968 & 3.537165 & -1.214021 & $\mathrm{H}$ & 2.295008 & -0.589678 & -1.505371 \\
\hline $\mathrm{H}$ & -2.849812 & 3.629755 & 0.164039 & $\mathrm{H}$ & -0.689595 & -1.476021 & 1.064226 \\
\hline $\mathrm{H}$ & -0.305731 & 5.198237 & -0.363494 & $\mathrm{H}$ & 4.493898 & -0.250004 & -0.622263 \\
\hline $\mathrm{H}$ & 0.137431 & 5.335559 & 2.151080 & $\mathrm{H}$ & 5.000818 & -1.399307 & 0.647907 \\
\hline $\mathrm{H}$ & 1.665271 & 4.079852 & 0.692620 & $\mathrm{H}$ & 4.348682 & -1.936145 & -2.258974 \\
\hline $\mathrm{H}$ & 1.316880 & 3.023660 & 2.070396 & $\mathrm{H}$ & 3.569766 & -4.293389 & -1.605755 \\
\hline $\mathrm{H}$ & -0.657510 & 3.482241 & 3.543193 & $\mathrm{H}$ & 1.966589 & -2.664722 & -2.506049 \\
\hline $\mathrm{H}$ & -2.082243 & 4.223827 & 2.816030 & $\mathrm{H}$ & 1.212814 & -3.376269 & -1.073950 \\
\hline $\mathrm{H}$ & -2.570150 & 2.062350 & 2.027234 & $\mathrm{H}$ & 2.580987 & -4.553224 & 0.625910 \\
\hline $\mathrm{H}$ & -0.897250 & 1.483184 & 2.326030 & $\mathrm{H}$ & 4.266283 & -4.086461 & 0.848475 \\
\hline $\mathrm{H}$ & -2.802766 & 5.824676 & 1.334285 & $\mathrm{H}$ & 3.432734 & -2.299737 & 2.130019 \\
\hline $\mathrm{H}$ & -2.595430 & 7.997128 & 0.231589 & $\mathrm{H}$ & 1.765965 & -2.556826 & 1.533928 \\
\hline $\mathrm{H}$ & -1.101681 & 7.404973 & -0.692497 & $\mathrm{H}$ & 6.171697 & -3.398480 & -0.246736 \\
\hline $\mathrm{H}$ & -4.042968 & -2.670330 & -1.302746 & $\mathrm{H}$ & 7.726103 & -3.365798 & -2.127397 \\
\hline $\mathrm{O}$ & -8.525185 & -2.750178 & -1.299008 & $\mathrm{H}$ & 6.480283 & -2.469150 & -3.165541 \\
\hline $\mathrm{H}$ & -2.854141 & -0.946797 & -0.686731 & $\mathrm{~N}$ & -0.272649 & 3.389149 & 0.215362 \\
\hline C & -9.741604 & -2.081506 & -1.095664 & 0 & -0.120395 & 2.220433 & -0.121541 \\
\hline $\mathrm{H}$ & -10.533023 & -2.809085 & -1.297507 & 0 & -1.353286 & 3.953910 & 0.276343 \\
\hline $\mathrm{H}$ & -9.855881 & -1.226681 & -1.781312 & $\mathrm{H}$ & -2.291774 & 1.295157 & -0.897443 \\
\hline $\mathrm{H}$ & -9.841398 & -1.721749 & -0.059798 & $C$ & -3.376602 & 1.292493 & -0.787234 \\
\hline $\mathrm{H}$ & 2.694508 & -1.230062 & -2.269645 & $C$ & -6.140879 & 1.336395 & -0.484499 \\
\hline C & 3.303051 & -2.128833 & -2.173223 & $\mathrm{C}$ & -3.991140 & 0.340750 & 0.036869 \\
\hline C & 4.857077 & -4.418654 & -1.943039 & $C$ & -4.134541 & 2.250446 & -1.448999 \\
\hline C & 3.528146 & -2.677151 & -0.904499 & $\mathrm{C}$ & -5.517904 & 2.276697 & -1.300721 \\
\hline C & 3.848400 & -2.713276 & -3.308665 & $C$ & -5.383630 & 0.376006 & 0.174882 \\
\hline C & 4.624538 & -3.864831 & -3.197977 & $\mathrm{H}$ & -3.633717 & 2.981671 & -2.081457 \\
\hline C & 4.319362 & -3.826254 & -0.808365 & $\mathrm{H}$ & -6.110025 & 3.029680 & -1.818585 \\
\hline $\mathrm{H}$ & 3.667595 & -2.264492 & -4.282814 & $\mathrm{H}$ & -5.870867 & -0.361657 & 0.813006 \\
\hline $\mathrm{H}$ & 5.048987 & -4.328064 & -4.086759 & $\mathrm{H}$ & -7.222625 & 1.352259 & -0.359387 \\
\hline $\mathrm{H}$ & 4.513559 & -4.254634 & 0.178031 & $C$ & -3.180675 & -0.691545 & 0.730136 \\
\hline $\mathrm{H}$ & 5.460994 & -5.318411 & -1.848568 & $\mathrm{~N}$ & -2.141106 & -0.424242 & 1.474844 \\
\hline C & 2.948906 & -2.065322 & 0.320699 & $\mathrm{C}$ & -3.516928 & -2.112302 & 0.545560 \\
\hline$N$ & 1.671988 & -1.874767 & 0.506518 & $\mathrm{C}$ & -4.001337 & -4.858655 & 0.126933 \\
\hline C & 3.869474 & -1.656324 & 1.393897 & $C$ & -3.005554 & -3.083351 & 1.424011 \\
\hline C & 5.601746 & -0.754195 & 3.424593 & $\mathrm{C}$ & -4.299058 & -2.554802 & -0.532410 \\
\hline C & 3.422522 & -1.504605 & 2.716655 & $C$ & -4.535636 & -3.909167 & -0.738435 \\
\hline C & 5.213588 & -1.359934 & 1.121093 & $\mathrm{C}$ & -3.237456 & -4.433841 & 1.213464 \\
\hline C & 6.066926 & -0.916514 & 2.123223 & $\mathrm{H}$ & -2.416859 & -2.748749 & 2.277862 \\
\hline C & 4.272174 & -1.056439 & 3.714974 & $\mathrm{H}$ & -4.696952 & -1.825299 & -1.236825 \\
\hline $\mathrm{H}$ & 2.389400 & -1.756543 & 2.950162 & $\mathrm{H}$ & -5.136063 & -4.223355 & -1.591824 \\
\hline $\mathrm{H}$ & 5.585169 & -1.452153 & 0.100834 & $\mathrm{H}$ & -2.828474 & -5.164912 & 1.910329 \\
\hline $\mathrm{H}$ & 7.102716 & -0.683853 & 1.879090 & $\mathrm{H}$ & -4.185946 & -5.919225 & -0.035882 \\
\hline $\mathrm{H}$ & 3.897683 & -0.951042 & 4.733195 & $C$ & -1.718220 & 0.817251 & 1.817479 \\
\hline $\mathrm{H}$ & 6.270483 & -0.405802 & 4.209512 & $\mathrm{H}$ & -2.243556 & 1.723989 & 1.511328 \\
\hline C & 0.692801 & -2.194675 & -0.382188 & $\mathrm{C}$ & -0.568931 & 0.988887 & 2.585040 \\
\hline $\mathrm{H}$ & 0.909553 & -2.634461 & -1.358526 & 0 & -0.048445 & 2.074831 & 2.923446 \\
\hline C & -0.656447 & -2.014558 & -0.069696 & 0 & 0.094848 & -0.200460 & 2.922943 \\
\hline $\mathrm{O}$ & -1.629081 & -2.227847 & -0.820374 & $\mathrm{C}$ & 0.205473 & -0.535124 & 4.320735 \\
\hline $\mathrm{O}$ & -0.901574 & -1.433891 & 1.183353 & $C$ & 0.539977 & -2.017414 & 4.308630 \\
\hline C & -1.487608 & -2.239324 & 2.227851 & $\mathrm{H}$ & 1.512828 & -2.188495 & 3.824961 \\
\hline C & -2.946496 & -2.551780 & 1.933620 & $\mathrm{H}$ & -0.226685 & -2.569716 & 3.747620 \\
\hline $\mathrm{H}$ & -3.048207 & -3.156815 & 1.024495 & $\mathrm{H}$ & 0.595635 & -2.417620 & 5.329594 \\
\hline
\end{tabular}




\begin{tabular}{llll|llll}
$\mathrm{H}$ & -3.518850 & -1.623978 & 1.794388 & $\mathrm{C}$ & -1.122957 & -0.302232 & 5.022732 \\
$\mathrm{H}$ & -3.387622 & -3.103140 & 2.777134 & $\mathrm{H}$ & -1.382807 & 0.763306 & 5.038374 \\
$\mathrm{C}$ & -1.383594 & -1.354597 & 3.455575 & $\mathrm{H}$ & -1.065200 & -0.655731 & 6.060704 \\
$\mathrm{H}$ & -0.338289 & -1.073451 & 3.641564 & $\mathrm{H}$ & -1.925969 & -0.844965 & 4.507469 \\
$\mathrm{H}$ & -1.778535 & -1.866289 & 4.341739 & $\mathrm{C}$ & 1.324603 & 0.264935 & 4.974049 \\
$\mathrm{H}$ & -1.968105 & -0.436180 & 3.301063 & $\mathrm{H}$ & 2.268517 & 0.113795 & 4.427897 \\
$\mathrm{C}$ & -0.683744 & -3.515684 & 2.398430 & $\mathrm{H}$ & 1.477923 & -0.065062 & 6.011296 \\
$\mathrm{H}$ & -0.767064 & -4.151900 & 1.508729 & $\mathrm{H}$ & 1.091012 & 1.334176 & 4.966098 \\
$\mathrm{H}$ & -1.053021 & -4.085006 & 3.261903 & & & &
\end{tabular}

\section{Synthetic Procedures and Structural Analysis}

\subsection{General Remarks}

All solvents were of reagent grade quality. All reagents were purchased from Sigma-Aldrich and $\mathrm{TCl}$ Chemicals and used without further purification. Column chromatography was carried out using Merck Kieselgel 60 (63-100 $\mu$ m mesh size), TLC was carried out on Merck Kieselgel F254 plates. Melting points were determined using a Boëtius M HMK hot-stage apparatus and were uncorrected. The NMR spectra were recorded on a Bruker Mercury 400 instrument. Chemical shifts are reported in ppm and are set to solvent residue peak. The splitting pattern of multiplets is described by abbreviations ( $s$ - singlet, $d$ - doublet, $t$ - triplet, $q$ - quartet, dd - doublet of doublets, $m$ - multiplet, $c$ - covered signal, $b$ - broad peak). J coupling constants values are reported in $\mathrm{Hz}$. Mass spectral analyses were performed with the ESI-TOF technique on a Mariner mass spectrometer from PerSeptive Biosystem.

\subsection{General Procedures}

Procedure $\mathbf{A}$ - for obtaining amides 1-21:

$$
\mathrm{H}_{2} \mathrm{~N}^{-\mathrm{R}} \quad \frac{\mathrm{BrCH}_{2} \mathrm{COBr}, \mathrm{K}_{2} \mathrm{CO}_{3}}{\mathrm{CH}_{2} \mathrm{Cl}_{2} / \mathrm{H}_{2} \mathrm{O}, 0^{\circ} \mathrm{C}, 1 \mathrm{~h}} \quad \mathrm{Br} \underset{\mathrm{H}}{\stackrel{\mathrm{H}-21}{\mathrm{O}}}
$$

To the solution of corresponding amine $(10 \mathrm{mmol})$ in DCM $(15 \mathrm{~mL})$ the aqueous $(20 \mathrm{~mL})$ solution of $\mathrm{K}_{2} \mathrm{CO}_{3}(2.1 \mathrm{~g}, 15 \mathrm{mmol})$ was added. Subsequently the mixture was cooled to $0{ }^{\circ} \mathrm{C}$ and the solution of bromoacetyl bromide $(1.1 \mathrm{~mL}, 15 \mathrm{mmol})$ in DCM $(3 \mathrm{~mL})$ was added dropwise. The reaction was controlled using TLC for about $1 \mathrm{~h}$. Then the phases were separated and the aqueous phase was extracted with DCM $(3 \times 15 \mathrm{~mL})$. The combined organic phases were dried over anhydrous $\mathrm{Na}_{2} \mathrm{SO}_{4}$ and then evaporated under vacuum. 
Procedure B - for obtaining catalysts 22-47:<smiles>[R]C1CCC([C@H](O)c2ccnc3ccccc23)NC1</smiles>

$\mathrm{R}_{2}=$ ethyl or vinyl

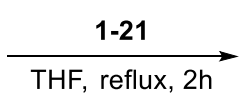

(n) aryl or alkyl

22-47

To the solution of cinchonidine $(1.0 \mathrm{~g}, 3.4 \mathrm{mmol})$ or hydrocynchonidine $(1.0 \mathrm{~g}, 3.4 \mathrm{mmol})$ in THF $(30 \mathrm{~mL})$ the corresponding amide $(3.4 \mathrm{mmol})$ was added. The mixture was refluxed for 1.5 $h$ and the reaction was controlled using TLC. Subsequently the solvent was evaporated under vacuum and the solid residue was dissolved in DCM $(2 \mathrm{~mL})$. The solution of crude catalyst was added dropwise to diethyl ether $(15 \mathrm{~mL})$. Then the precipitate was filtered, washed with diethyl ether $(10 \mathrm{~mL})$ and dried. Catalysts $\mathbf{2 2 - 4 7}$ were obtained in a form of solids.

Procedure $\mathbf{C}$ - for alkylation of $\mathrm{N}$-(diphenylmethylene)glycine tert-butyl ester:<smiles>CC(C)(C)OC(=O)CN=C(c1ccccc1)c1ccccc1</smiles>

48<smiles>[R]c1ccccc1CBr</smiles>

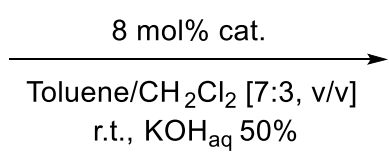

r.t., $\mathrm{KOH}_{\mathrm{aq}} 50 \%$<smiles>[R]C1=CC=CC1CC(=NC(=O)c1ccccc1)C(=O)OC(C)(C)C</smiles>

49-55

To the solution of catalyst $\mathbf{2 2 - 4 7}(0,016 \mathrm{mmol})$ and $\mathrm{N}$-(diphenylmethylene)glycine tert-butyl ester $48(59 \mathrm{mg}, 0.2 \mathrm{mmol})$ in toluene $/ \mathrm{CH}_{2} \mathrm{Cl}_{2}[7: 3, \mathrm{v} / \mathrm{v}](1 \mathrm{~mL})$ the corresponding benzyl bromide $(1.0 \mathrm{mmol})$ was added. Then $50 \%$ aqueous solution of $\mathrm{KOH}(0.6 \mathrm{~mL})$ was added. The heterogeneous mixture was cooled to $0{ }^{\circ} \mathrm{C}$. The reaction was controlled using TLC for $8-12 \mathrm{~h}$. Subsequently the solvents were evaporated under vacuum and the residue was purified employing column chromatography and using hexane/ethyl acetate [99:1 $\rightarrow$ 95:5, v/v] mixture as eluent. Products 49-55 were obtained in a form of colorless oils.

\subsection{Substance analysis}

\section{Compound 1}

Following Procedure $\mathbf{A}$ and using aniline $(0.9 \mathrm{~mL}, 10 \mathrm{mmol})$, the product $1(2.0 \mathrm{~g}, 9.2 \mathrm{mmol}, \mathbf{9 2} \%)$ was obtained as colorless solid $\left(\mathrm{mp} 123-124^{\circ} \mathrm{C}\right)$.

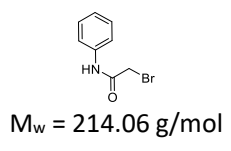

${ }^{1} \mathrm{H}$ NMR (400 MHz, DMSO- $\left.d_{6}\right)$

${ }^{13} \mathrm{C}$ NMR $\left(100 \mathrm{MHz}\right.$, DMSO- $\left.d_{6}\right)$ $\delta 10.35(\mathrm{~s}, 1 \mathrm{H}), 7.59(\mathrm{~d}, J=7.9 \mathrm{~Hz}, 2 \mathrm{H}), 7.33(\mathrm{t}, J=7.9 \mathrm{~Hz}, 2 \mathrm{H}), 7.08(\mathrm{t}, \mathrm{J}=7.4 \mathrm{~Hz}, 1 \mathrm{H}), 4.04(\mathrm{~s}$, $2 \mathrm{H})$.

$\delta 164.7,138.6,128.8,123.8,119.2,30.4$ 


\section{Compound 2}

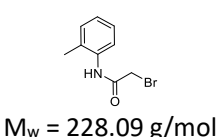

Following Procedure $\mathbf{A}$ and using o-toluidine $(1.1 \mathrm{~mL}, 10 \mathrm{mmol})$, the product $2(2.2 \mathrm{~g}, 9.8 \mathrm{mmol}$, 98\%) was obtained as colorless solid $\left(\mathrm{mp} 102-103^{\circ} \mathrm{C}\right)$.

$M_{w}=228.09 \mathrm{~g} / \mathrm{mol}$

${ }^{1} \mathrm{H}$ NMR $\left(400 \mathrm{MHz}, \mathrm{DMSO}-d_{6}\right)$

$\delta 9.70(\mathrm{~s}, 1 \mathrm{H}), 7.39(\mathrm{~d}, J=7.7 \mathrm{~Hz}, 1 \mathrm{H}), 7.23(\mathrm{~d}, J=7.4 \mathrm{~Hz}, 1 \mathrm{H}), 7.18(\mathrm{td}, J=7.7,1.3 \mathrm{~Hz}, 1 \mathrm{H}), 7.11$ (td, $J=7.4,1.3 \mathrm{~Hz}, 1 \mathrm{H}), 4.09(\mathrm{~s}, 2 \mathrm{H}), 2.21(\mathrm{~s}, 3 \mathrm{H})$.

${ }^{13} \mathrm{C}$ NMR $\left(100 \mathrm{MHz}, \mathrm{DMSO}-d_{6}\right)$

$\delta 165.0,135.6,131.9,130.4,126.0,125.6,124.8,29.9,17.6$

\section{Compound 3}

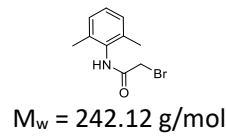

Following Procedure $\mathbf{A}$ and using 2,6-dimethylaniline $(1.2 \mathrm{~mL}, 10 \mathrm{mmol})$, the product 3 (2.4 g, $9.9 \mathrm{mmol}, 99 \%)$ was obtained as colorless solid ( $\mathrm{mp} 150-151^{\circ} \mathrm{C}$ ).

\section{Compound 4}

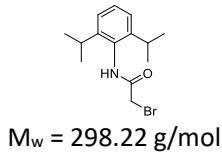

Following Procedure A and using 2,6-diisopropylaniline $(1.9 \mathrm{~mL}, 10 \mathrm{mmol})$, the product 4 (2.8 $\mathrm{g}$, $9.3 \mathrm{mmol}, \mathbf{9 3} \%$ ) was obtained as colorless solid ( $\mathrm{mp} 159-160{ }^{\circ} \mathrm{C}$ ).

${ }^{1} \mathrm{H}$ NMR (400 MHz, DMSO- $\left.d_{6}\right)$

$\delta 9.66(\mathrm{~s}, 1 \mathrm{H}), 7.27(\mathrm{dd}, J=8.1,7.2 \mathrm{~Hz}, 1 \mathrm{H}), 7.16(\mathrm{~d}, J=7.7 \mathrm{~Hz}, 2 \mathrm{H}), 4.06(\mathrm{~s}, 2 \mathrm{H}), 3.08$ (hept, $J=$ $6.8 \mathrm{~Hz}, 2 \mathrm{H}), 1.12$ (d, J = 6.7 Hz, 12H).

${ }^{13} \mathrm{C}$ NMR $\left(100 \mathrm{MHz}, \mathrm{DMSO}-d_{6}\right)$

$\delta 165.8,145.9,131.6,127.7,122.9,29.2,27.7,23.7,23.2$.

\section{Compound 5}

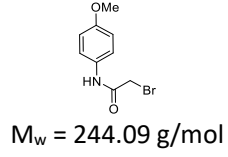

${ }^{1} \mathbf{H}$ NMR (400 MHz, DMSO- $\left.d_{6}\right)$ ${ }^{13} \mathrm{C}$ NMR $\left(100 \mathrm{MHz}, \mathrm{DMSO}-d_{6}\right)$
Following Procedure $\mathbf{A}$ and using $p$-anisidine $(1.2 \mathrm{~g}, 10 \mathrm{mmol})$, the product $\mathbf{5}(2.3 \mathrm{~g}, 9.4 \mathrm{mmol}$, 94\%) was obtained as pale brown solid ( $\left.\mathrm{mp} 120-121^{\circ} \mathrm{C}\right)$.

$\delta 10.22(\mathrm{~s}, 1 \mathrm{H}), 7.52-7.47(\mathrm{~m}, 2 \mathrm{H}), 6.92-6.87(\mathrm{~m}, 2 \mathrm{H}), 4.00(\mathrm{~s}, 2 \mathrm{H})$.

$\delta 164.2,155.6,131.7,120.7,113.9,55.2,30.4$.

\section{Compound 6}

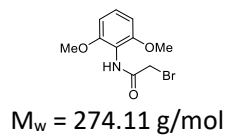

${ }^{1} \mathrm{H}$ NMR $\left(400 \mathrm{MHz}\right.$, DMSO- $\left.d_{6}\right)$ ${ }^{13} \mathrm{C}$ NMR $\left(100 \mathrm{MHz}\right.$, DMSO- $\left.d_{6}\right)$
Following Procedure A and using 2,6-dimethoxyaniline $(1.5 \mathrm{~g}, 10 \mathrm{mmol})$, the product 6 ( $2.6 \mathrm{~g}$, $9.4 \mathrm{mmol}, \mathbf{9 4 \%}$ ) was obtained as brown solid ( $\mathrm{mp} 160-161^{\circ} \mathrm{C}$ ).

$\delta 9.26(\mathrm{~s}, 1 \mathrm{H}), 7.22(\mathrm{t}, J=8.4 \mathrm{~Hz}, 1 \mathrm{H}), 6.68(\mathrm{~d}, J=8.4 \mathrm{~Hz}, 2 \mathrm{H}), 4.02(\mathrm{~s}, 2 \mathrm{H}), 3.74(\mathrm{~s}, 6 \mathrm{H})$. $\delta 164.4,155.7,127.8,114.0,104.5,55.8,29.5$. 


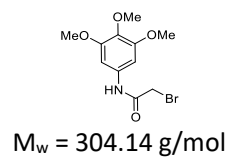

${ }^{1} \mathrm{H}$ NMR $\left(400 \mathrm{MHz}\right.$, DMSO- $\left.d_{6}\right)$ ${ }^{13} \mathrm{C}$ NMR $\left(100 \mathrm{MHz}\right.$, DMSO- $\left.d_{6}\right)$
Following Procedure A and using 3,4,5-trimethoxyaniline $(1.8 \mathrm{~g}, 10 \mathrm{mmol})$, the product 7 ( $2.9 \mathrm{~g}$, $9.4 \mathrm{mmol}, \mathbf{9 4 \%}$ ) was obtained as colorless solid ( $\left.\mathrm{mp} 125-126^{\circ} \mathrm{C}\right)$.

$\delta 10.29(\mathrm{~s}, 1 \mathrm{H}), 6.96(\mathrm{~s}, 2 \mathrm{H}), 4.01(\mathrm{~s}, 2 \mathrm{H}), 3.75(\mathrm{~s}, 6 \mathrm{H}), 3.62(\mathrm{~s}, 3 \mathrm{H})$ $\delta 164.5,152.7,134.7,133.8,97.0,60.1,55.7,30.4$.

\section{Compound 8}

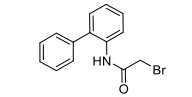

$\mathrm{M}_{\mathrm{w}}=290.16 \mathrm{~g} / \mathrm{mol}$

${ }^{1} \mathrm{H}$ NMR $\left(400 \mathrm{MHz}\right.$, DMSO- $\left.d_{6}\right)$

${ }^{13} \mathrm{C}$ NMR $\left(100 \mathrm{MHz}\right.$, DMSO- $\left.d_{6}\right)$
Following Procedure $\mathbf{A}$ and using 2-phenylaniline $(1.7 \mathrm{~mL}, 10 \mathrm{mmol})$, the product $8(2.7 \mathrm{~g}, 9.2$ $\mathrm{mmol}, \mathbf{9 2} \%$ ) was obtained as colorless solid ( $\left.\mathrm{mp} 86-87^{\circ} \mathrm{C}\right)$.

$\delta 9.64(\mathrm{~s}, 1 \mathrm{H}), 7.54(\mathrm{~d}, J=7.7 \mathrm{~Hz}, 1 \mathrm{H}), 7.48-7.43(\mathrm{~m}, 2 \mathrm{H}), 7.41-7.30(\mathrm{~m}, 6 \mathrm{H}), 3.95(\mathrm{~s}, 2 \mathrm{H})$.

$\delta 165.1,138.3,136.3,134.0,130.3,128.8,128.4,127.8,127.4,126.5,126.3,29.6$.

\section{Compound 9}

Following Procedure $A$ and using 4-nitroaniline $(1.4 \mathrm{~g}, 10 \mathrm{mmol})$, the pro was obtained as yellow solid (mp 165-166 $\left.{ }^{\circ} \mathrm{C}\right)$.
$96 \%)$ w
$\mathrm{M}_{\mathrm{w}}=259.06 \mathrm{~g} / \mathrm{mol}$
$\left.100 \mathrm{MHz}, \mathrm{DMSO}-d_{6}\right)$
$\delta 10.95(\mathrm{~s}, 1 \mathrm{H}), 8.26-8.22(\mathrm{~m}, 2 \mathrm{H}), 7.85-7.81(\mathrm{~m}, 2 \mathrm{H}), 4.11(\mathrm{~s}, 2 \mathrm{H})$.
$\delta 165.7,144.6,142.6,125.0,119.0,30.1$.

${ }^{1} \mathrm{H}$ NMR (400 MHz, DMSO- $\left.d_{6}\right)$ $\delta 165.7,144.6,142.6,125.0,119.0,30.1$

\section{Compound 10}

Following Procedure $\mathbf{A}$ and using 2-nitroaniline $(1.4 \mathrm{~g}, 10 \mathrm{mmol})$, the product 10 (2.5 g, 9.7 $\mathrm{mmol}, \mathbf{9 7 \%})$ was obtained as yellowish solid $\left(\mathrm{mp} 62-63^{\circ} \mathrm{C}\right)$.

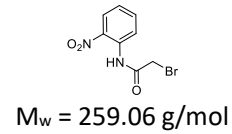

${ }^{1} \mathrm{H}$ NMR $\left(400 \mathrm{MHz}, \mathrm{DMSO}-d_{6}\right)$

${ }^{13} \mathrm{C}$ NMR $\left(100 \mathrm{MHz}\right.$, DMSO- $\left.d_{6}\right)$ $\delta 10.67(\mathrm{~s}, 1 \mathrm{H}), 8.00(\mathrm{dd}, J=8.2,0.8 \mathrm{~Hz}, 1 \mathrm{H}), 7.76-7.69(\mathrm{~m}, 2 \mathrm{H}), 7.41$ (ddd, $J=8.2,6.5,2.3 \mathrm{~Hz}$, $1 \mathrm{H}), 4.14(\mathrm{~s}, 2 \mathrm{H})$.

$\delta 165.1,142.2,134.1,130.6,125.8,125.2,125.0,29.4$.

\section{Compound $\mathbf{1 1}$}

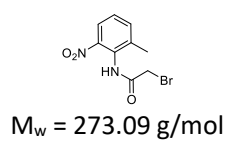

${ }^{1} \mathrm{H}$ NMR $(400 \mathrm{MHz}$, DMSO-d6)

${ }^{13} \mathrm{C}$ NMR $\left(100 \mathrm{MHz}, \mathrm{DMSO}-d_{6}\right)$
Following Procedure A and using 2-methyl-6-nitroaniline (1.5 g, $10 \mathrm{mmol})$, the product 11 (2.6 $\mathrm{g}, 9.7 \mathrm{mmol}, \mathbf{9 7 \%})$ was obtained as yellowish solid ( $\left.\mathrm{mp} 140-141^{\circ} \mathrm{C}\right)$.

$\delta 10.28(\mathrm{~s}, 1 \mathrm{H}), 7.77$ (dd, $J=7.9,0.8 \mathrm{~Hz}, 1 \mathrm{H}), 7.63(\mathrm{dd}, J=7.9,0.5 \mathrm{~Hz}, 1 \mathrm{H}), 7.41(\mathrm{t}, J=7.9 \mathrm{~Hz}, 1 \mathrm{H})$, $4.08(\mathrm{~s}, 2 \mathrm{H}), 2.30(\mathrm{~s}, 3 \mathrm{H})$.

$\delta 165.1,146.5,137.2,135.0,127.9,127.1,122.2,28.7,17.5$. 


\section{Compound 12}

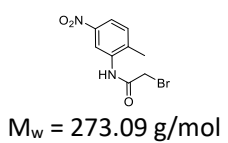

Following Procedure A and using 2-methyl-5-nitroaniline (1.5 g, $10 \mathrm{mmol})$, the product 12 (2.6 $\mathrm{g}, 9.4 \mathrm{mmol}, \mathbf{9 4 \%}$ ) was obtained as colorless solid ( $\left.\mathrm{mp} 150-151{ }^{\circ} \mathrm{C}\right)$.

$M_{w}=273.09 \mathrm{~g} / \mathrm{mol}$

${ }^{1} \mathrm{H}$ NMR $\left(400 \mathrm{MHz}, \mathrm{DMSO}-d_{6}\right)$

$\delta 9.99(\mathrm{~s}, 1 \mathrm{H}), 8.42(\mathrm{~d}, J=1.9 \mathrm{~Hz}, 1 \mathrm{H}), 7.97(\mathrm{dd}, J=8.4,1.9 \mathrm{~Hz}, 1 \mathrm{H}), 7.52(\mathrm{~d}, J=8.4 \mathrm{~Hz}, 1 \mathrm{H}), 4.18$ $(\mathrm{s}, 2 \mathrm{H}), 2.36(\mathrm{~s}, 3 \mathrm{H})$.

${ }^{13} \mathrm{C}$ NMR $\left(100 \mathrm{MHz}, \mathrm{DMSO}-d_{6}\right)$

$\delta 165.7,145.7,139.2,136.6,131.5,119.8,118.4,29.8,18.0$.

\section{Compound 13}

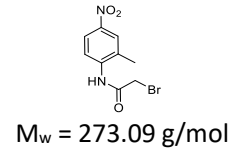

Following Procedure A and using 2-methyl-4-nitroaniline $(1.5 \mathrm{~g}, 10 \mathrm{mmol})$, the product $13(2.7$ $\mathrm{g}, 9.8 \mathrm{mmol}, \mathbf{9 8 \%})$ was obtained as colorless solid ( $\left.\mathrm{mp} 129-130^{\circ} \mathrm{C}\right)$.

${ }^{1} \mathrm{H}$ NMR $\left(400 \mathrm{MHz}, \mathrm{DMSO}-d_{6}\right)$

$\delta 9.93(\mathrm{~s}, 1 \mathrm{H}), 8.14(\mathrm{~d}, J=2.3 \mathrm{~Hz}, 1 \mathrm{H}), 8.08(\mathrm{dd}, J=8.9,2.6 \mathrm{~Hz}, 1 \mathrm{H}), 7.90(\mathrm{~d}, J=8.9 \mathrm{~Hz}, 1 \mathrm{H}), 4.20$ $(\mathrm{s}, 2 \mathrm{H}), 2.36(\mathrm{~s}, 3 \mathrm{H})$.

${ }^{13} \mathrm{C}$ NMR $\left(100 \mathrm{MHz}\right.$, DMSO- $\left.d_{6}\right)$

$\delta 165.6,143.6,142.1,131.6,125.4,123.5,121.7,29.9,17.7$.

\section{Compound 14}

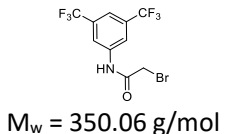

${ }^{1} \mathrm{H}$ NMR (400 MHz, DMSO- $d_{6}$ ) ${ }^{13} \mathrm{C}$ NMR $\left(100 \mathrm{MHz}\right.$, DMSO- $\left.d_{6}\right)$
Following Procedure $\mathbf{A}$ and using 3,5-bis(trifluoromethyl)aniline (1.6 mL, $10 \mathrm{mmol}$ ), the product 14 (3.3 g, $9.5 \mathrm{mmol}, 95 \%)$ was obtained as colorless solid ( $\left.\mathrm{mp} 95-96^{\circ} \mathrm{C}\right)$.

$\delta 11.02(\mathrm{~s}, 1 \mathrm{H}), 8.23(\mathrm{~s}, 2 \mathrm{H}), 7.78(\mathrm{~s}, 1 \mathrm{H}), 4.10(\mathrm{~s}, 2 \mathrm{H})$.

$\delta 165.9,140.4,130.9(q, J=33.0 \mathrm{~Hz}), 124.4,121.7,118.9,116.6,29.9$

\section{Compound 15}

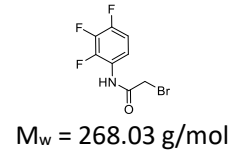

${ }^{1} \mathrm{H}$ NMR (400 MHz, DMSO- $d_{6}$ )

${ }^{13} \mathrm{C}$ NMR (100 MHz, DMSO- $\left.d_{6}\right)$
Following Procedure $\mathbf{A}$ and using 2,3,4-Trifluoroaniline $(1.1 \mathrm{~mL}, 10 \mathrm{mmol})$, the product $15(2.5$ $\mathrm{g}, 9.5 \mathrm{mmol}, \mathbf{9 5 \%})$ was obtained as colorless solid $\left(\mathrm{mp} 85-86^{\circ} \mathrm{C}\right)$.

$\delta 10.36(\mathrm{~s}, 1 \mathrm{H}), 7.67-7.58(\mathrm{~m}, 1 \mathrm{H}), 7.35-7.26(\mathrm{~m}, 1 \mathrm{H}), 4.14(\mathrm{~s}, 2 \mathrm{H})$.

$\delta 165.5,123.5,123.4(x 3), 118.77(x 3), 112.0(x 2), 111.8(x 2), 29.3$.

\section{Compound 16}

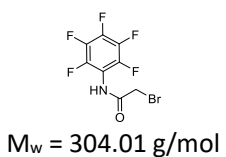

${ }^{1} \mathbf{H}$ NMR $\left(400 \mathrm{MHz}, \mathrm{DMSO}-d_{6}\right)$ ${ }^{13} \mathrm{C}$ NMR (100 MHz, DMSO- $\left.d_{6}\right)$
Following Procedure $\mathbf{A}$ and using 2,3,4,5,6-pentafluoroaniline (1.8 g, $10 \mathrm{mmol}$ ), the product 16 ( $2.9 \mathrm{~g}, 9.4 \mathrm{mmol}, 94 \%)$ was obtained as colorless solid (mp $201-202{ }^{\circ} \mathrm{C}$ ).

$10.52(\mathrm{~s}, 1 \mathrm{H}), 4.14(\mathrm{~s}, 2 \mathrm{H})$

$\delta 165.5,28.2$. 


\section{Compound 17}

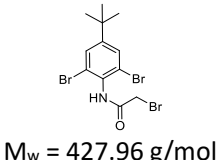

Following Procedure A and using 2,6-dibromo-4-tert-butyl aniline (3.1 g, $10 \mathrm{mmol}$ ), the product 17 (4.0 g, $9.4 \mathrm{mmol}, 94 \%)$ was obtained as colorless solid ( $\mathrm{mp} 196-197{ }^{\circ} \mathrm{C}$ ).

$\mathrm{M}_{\mathrm{w}}=427.96 \mathrm{~g} / \mathrm{mol}$

${ }^{1} \mathrm{H}$ NMR (400 MHz, DMSO- $\left.d_{6}\right)$ ${ }^{13} \mathrm{C}$ NMR $\left(100 \mathrm{MHz}\right.$, DMSO- $\left.d_{6}\right)$ HRMS ESI $(\mathrm{m} / \mathrm{z})$ $\delta 10.23(\mathrm{~s}, 1 \mathrm{H}), 7.67(\mathrm{~s}, 2 \mathrm{H}), 4.07(\mathrm{~s}, 2 \mathrm{H}), 1.28(\mathrm{~s}, 9 \mathrm{H})$

$\delta 164.6,153.5,132.3,129.2,123.6,34.6,30.6,28.7$.

Calc. for $\mathrm{C}_{12} \mathrm{H}_{14} \mathrm{NONaBr}_{3}[\mathrm{M}+\mathrm{Na}]^{+}:$:447.8523, found: 447.8510 .

\section{Compound 18}

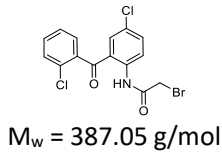

Following Procedure $\mathbf{A}$ and using 2-amino-2',5-dichlorobenzophenone (2.7 $\mathrm{g}, 10 \mathrm{mmol})$, the product 18 (3.6 g, $9.4 \mathrm{mmol}, \mathbf{9 4 \%})$ was obtained as colorless solid ( $\mathrm{mp} 124-125^{\circ} \mathrm{C}$ ).

${ }^{1} \mathrm{H}$ NMR $\left(400 \mathrm{MHz}, \mathrm{DMSO}-d_{6}\right)$

$\delta 11.10(\mathrm{~s}, 1 \mathrm{H}), 8.11(\mathrm{~d}, J=8.9 \mathrm{~Hz}, 1 \mathrm{H}), 7.75(\mathrm{dd}, J=8.9,2.5 \mathrm{~Hz}, 1 \mathrm{H}), 7.59(\mathrm{dd}, J=6.5,4.2 \mathrm{~Hz}, 3 \mathrm{H})$, $7.53-7.47(\mathrm{~m}, 1 \mathrm{H}), 7.31(\mathrm{~d}, J=2.5 \mathrm{~Hz}, 1 \mathrm{H}), 4.11(\mathrm{~s}, 2 \mathrm{H})$.

${ }^{13} \mathrm{C}$ NMR $\left(100 \mathrm{MHz}\right.$, DMSO- $\left.d_{6}\right)$

\section{Compound 19}

Following Procedure $\mathbf{A}$ and using 3-amino-2-chloropyridine $(1.3 \mathrm{~g}, 10 \mathrm{mmol})$, the product 19 ( $2.3 \mathrm{~g}, 9.1 \mathrm{mmol}, 91 \%)$ was obtained as colorless solid $\left(\mathrm{mp} 76-77^{\circ} \mathrm{C}\right)$.

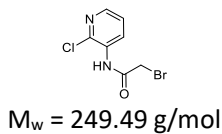

${ }^{1} \mathrm{H}$ NMR (400 MHz, DMSO- $\left.d_{6}\right)$

$\delta 10.08(\mathrm{~s}, 1 \mathrm{H}), 8.24(\mathrm{dd}, J=4.7,1.8 \mathrm{~Hz}, 1 \mathrm{H}), 8.18(\mathrm{dd}, J=8.0,1.8 \mathrm{~Hz}, 1 \mathrm{H}), 7.46(\mathrm{dd}, J=8.0,4.7$ $\mathrm{Hz}, 1 \mathrm{H}), 4.21(\mathrm{~s}, 2 \mathrm{H})$.

${ }^{13} \mathrm{C}$ NMR $\left(100 \mathrm{MHz}\right.$, DMSO- $\left.d_{6}\right)$

$\delta 165.8,145.8,143.1,133.9,131.4,123.4,29.5$.

\section{Compound 20}

Following Procedure $\mathbf{A}$ and using amantadine $(1.5 \mathrm{~g}, 10 \mathrm{mmol})$, the product $20(2.5 \mathrm{~g}, 9.3 \mathrm{mmol}$, 93\%) was obtained as colorless solid ( $\mathrm{mp} 123-124^{\circ} \mathrm{C}$ ).

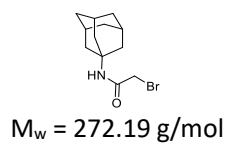

${ }^{1} \mathrm{H}$ NMR $\left(400 \mathrm{MHz}, \mathrm{DMSO}-d_{6}\right)$

${ }^{13} \mathrm{C}$ NMR (100 MHz, DMSO- $\left.d_{6}\right)$ $\delta 7.72(\mathrm{~s}, 1 \mathrm{H}), 3.76(\mathrm{~s}, 2 \mathrm{H}), 2.01(\mathrm{bs}, 3 \mathrm{H}), 1.91(\mathrm{~d}, J=3.0 \mathrm{~Hz}, 6 \mathrm{H}), 1.62(\mathrm{t}, J=3.0 \mathrm{~Hz}, 6 \mathrm{H})$.

$\delta 164.9,51.1,40.6,35.9,30.8,28.7$.

\section{Compound $\mathbf{2 1}$}

Following Procedure A and using tert-butylamine $(1.0 \mathrm{~mL}, 10 \mathrm{mmol})$, the product $21(1.9 \mathrm{~g}, 9.8$ $\mathrm{mmol}, \mathbf{9 8 \%}$ ) was obtained as colorless solid ( $\mathrm{mp} 94-95^{\circ} \mathrm{C}$ ). 


\section{Compound 22}

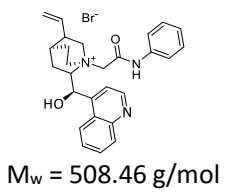

${ }^{1} \mathrm{H}$ NMR $\left(400 \mathrm{MHz}, \mathrm{DMSO}-d_{6}\right)$

${ }^{13} \mathrm{C}$ NMR $\left(100 \mathrm{MHz}, \mathrm{DMSO}-d_{6}\right)$

HRMS ESI $(\mathrm{m} / \mathrm{z})$
Following Procedure B and using 1 (0.7 g, $3.4 \mathrm{mmol})$, the product $22(1.4 \mathrm{~g}, 3.3 \mathrm{mmol}, \mathbf{9 6 \%})$ was obtained as colorless solid $\left(\mathrm{mp} 152-153^{\circ} \mathrm{C} ;[\alpha]_{\mathrm{D}}^{20}=-68.6(c=1.0, \mathrm{MeOH})\right)$.

\section{Compound 23}

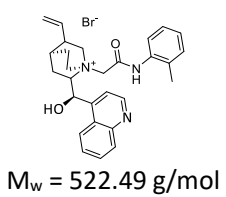

${ }^{13} \mathrm{C}$ NMR $\left(100 \mathrm{MHz}, \mathrm{DMSO}-d_{6}\right)$

HRMS ESI $(m / z)$
Following Procedure B and using $2(0.8 \mathrm{~g}, 3.4 \mathrm{mmol})$, the product $23(1.7 \mathrm{~g}, 3.3 \mathrm{mmol}, \mathbf{9 8 \%})$ was obtained as pale brown solid $\left(\mathrm{mp} 143-144^{\circ} \mathrm{C} ;[\alpha]_{\mathrm{D}}^{20}=-41.4(c=1.0, \mathrm{MeOH})\right)$ $\delta 11.17(\mathrm{~s}, 1 \mathrm{H}), 8.96(\mathrm{~d}, J=3.4 \mathrm{~Hz}, 1 \mathrm{H}), 8.17(\mathrm{~d}, J=8.0 \mathrm{~Hz}, 1 \mathrm{H}), 8.06(\mathrm{~d}, J=8.1 \mathrm{~Hz}, 1 \mathrm{H}), 7.86-$ $7.71(\mathrm{~m}, 4 \mathrm{H}), 7.54(\mathrm{t}, J=7.1 \mathrm{~Hz}, 1 \mathrm{H}), 7.44(\mathrm{t}, J=7.2 \mathrm{~Hz}, 2 \mathrm{H}), 7.19(\mathrm{t}, J=6.8 \mathrm{~Hz}, 1 \mathrm{H}), 6.75(\mathrm{~s}, 1 \mathrm{H})$ $6.16(\mathrm{~s}, 1 \mathrm{H}), 5.75-5.60(\mathrm{~m}, 1 \mathrm{H}), 5.20(\mathrm{~d}, J=17.3 \mathrm{~Hz}, 1 \mathrm{H}), 4.96(\mathrm{dd}, J=24.1,13.1 \mathrm{~Hz}, 2 \mathrm{H}), 4.78$ $(\mathrm{d}, J=15.6 \mathrm{~Hz}, 1 \mathrm{H}), 4.44(\mathrm{bs}, 1 \mathrm{H}), 4.37(\mathrm{~d}, J=12.1 \mathrm{~Hz}, 1 \mathrm{H}), 4.28(\mathrm{t}, J=8.2 \mathrm{~Hz}, 1 \mathrm{H}), 4.00(\mathrm{t}, J=11.1$ $\mathrm{Hz}, 1 \mathrm{H}), 3.82(\mathrm{t}, J=9.3 \mathrm{~Hz}, 1 \mathrm{H}), 2.87(\mathrm{bs}, 1 \mathrm{H}), 2.17-1.93(\mathrm{~m}, 4 \mathrm{H}), 1.17(\mathrm{t}, J=10.2 \mathrm{~Hz}, 1 \mathrm{H})$. $\delta 162.6,150.1,147.5,144.8,138.1,137.7,129.9,129.4,129.0,127.0,124.6,124.1,122.9,120.1$, $119.7,115.9,65.0,64.7,60.6,59.4,55.5,36.9,25.3,24.7,20.8$

Calc. for $\mathrm{C}_{27} \mathrm{H}_{30} \mathrm{~N}_{3} \mathrm{O}_{2}[\mathrm{M}]^{+}$: 428.2338, found: 428.2336 .
${ }^{1} \mathrm{H}$ NMR (400 MHz, DMSO- $\left.d_{6}\right)$

\section{Compound 24}

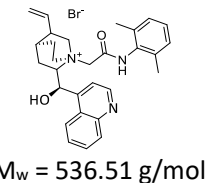

${ }^{1} \mathrm{H}$ NMR (400 MHz, DMSO- $\left.d_{6}\right)$

${ }^{13} \mathrm{C}$ NMR $\left(100 \mathrm{MHz}, \mathrm{DMSO}-d_{6}\right)$

HRMS ESI $(m / z)$
Following Procedure B and using $3(0.8 \mathrm{~g}, 3.4 \mathrm{mmol})$, the product $24(1.7 \mathrm{~g}, 3.3 \mathrm{mmol}, \mathbf{9 7 \%})$ was obtained as colorless solid $\left(\mathrm{mp} 217-218^{\circ} \mathrm{C} ;[\alpha]_{\mathrm{D}}^{20}=-53.4(c=1.0, \mathrm{MeOH})\right)$.

$\delta 10.45(\mathrm{~s}, 1 \mathrm{H}), 8.97(\mathrm{~d}, J=4.5 \mathrm{~Hz}, 1 \mathrm{H}), 8.22(\mathrm{~d}, J=8.5 \mathrm{~Hz}, 1 \mathrm{H}), 8.06(\mathrm{~d}, J=8.4 \mathrm{~Hz}, 1 \mathrm{H}), 7.82-$ $7.73(\mathrm{~m}, 2 \mathrm{H}), 7.50(\mathrm{t}, J=7.6 \mathrm{~Hz}, 1 \mathrm{H}), 7.20-7.11(\mathrm{~m}, 3 \mathrm{H}), 6.88(\mathrm{~d}, J=4.2 \mathrm{~Hz}, 1 \mathrm{H}), 6.16(\mathrm{bs}, 1 \mathrm{H})$, $5.72-5.60(\mathrm{~m}, 1 \mathrm{H}), 5.14(\mathrm{dd}, J=28.9,16.7 \mathrm{~Hz}, 2 \mathrm{H}), 4.89(\mathrm{t}, J=14.7 \mathrm{~Hz}, 2 \mathrm{H}), 4.49(\mathrm{t}, J=8.6 \mathrm{~Hz}$, $1 \mathrm{H}), 4.40(\mathrm{t}, J=9.2 \mathrm{~Hz}, 1 \mathrm{H}), 4.28(\mathrm{~d}, J=12.4 \mathrm{~Hz}, 1 \mathrm{H}), 4.05(\mathrm{t}, J=11.4 \mathrm{~Hz}, 1 \mathrm{H}), 3.83(\mathrm{t}, J=10.7 \mathrm{~Hz}$, $1 \mathrm{H}), 2.89$ (bs, 1H), 2.25 (s, 6H), 2.10 (bs, $2 \mathrm{H}), 2.02-1.92(\mathrm{~m}, 2 \mathrm{H}), 1.09(\mathrm{t}, J=10.9 \mathrm{~Hz}, 1 \mathrm{H})$. $\delta 162.9,150.1,147.5,144.8,138.0,135.1,133.3,129.8,129.4,127.9,127.2,126.9,124.2,123.2$ $120.0,115.7,65.3,64.4,60.0,58.5,55.5,36.8,25.3,24.7,21.0,18.3$

Calc. for $\mathrm{C}_{29} \mathrm{H}_{34} \mathrm{~N}_{3} \mathrm{O}_{2}[\mathrm{M}]^{+}: 456.2651$, found: 456.2640 . 


\section{Compound 25}

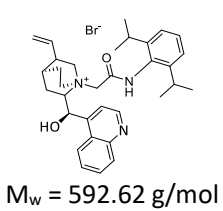

Following Procedure $B$ and using $4(1.0 \mathrm{~g}, 3.4 \mathrm{mmol})$, the product $25(1.7 \mathrm{~g}, 3.1 \mathrm{mmol}, \mathbf{9 2} \%)$ was obtained as colorless solid $\left(\mathrm{mp} 225-226^{\circ} \mathrm{C} ;[\alpha]_{\mathrm{D}}^{20}=-37.7(c=1.0, \mathrm{MeOH})\right)$

${ }^{13} \mathrm{C}$ NMR $\left(100 \mathrm{MHz}\right.$, DMSO- $\left.d_{6}\right)$

HRMS ESI $(m / z)$

$\delta 10.35(\mathrm{~s}, 1 \mathrm{H}), 8.97(\mathrm{~d}, J=4.5 \mathrm{~Hz}, 1 \mathrm{H}), 8.25(\mathrm{~d}, J=8.4 \mathrm{~Hz}, 1 \mathrm{H}), 8.06(\mathrm{~d}, J=8.2 \mathrm{~Hz}, 1 \mathrm{H}), 7.79(\mathrm{~d}, J$ $=4.5 \mathrm{~Hz}, 1 \mathrm{H}), 7.75(\mathrm{t}, J=7.5 \mathrm{~Hz}, 1 \mathrm{H}), 7.53(\mathrm{t}, J=7.3 \mathrm{~Hz}, 1 \mathrm{H}), 7.35(\mathrm{t}, J=7.7 \mathrm{~Hz}, 1 \mathrm{H}), 7.23(\mathrm{dd}, J=$ $11.8,7.8 \mathrm{~Hz}, 2 \mathrm{H}), 6.92(\mathrm{~d}, J=4.3 \mathrm{~Hz}, 1 \mathrm{H}), 6.10(\mathrm{t}, J=3.6 \mathrm{~Hz}, 1 \mathrm{H}), 5.71-5.62(\mathrm{~m}, 1 \mathrm{H}), 5.12(\mathrm{dd}, J$ $=16.6,13.8 \mathrm{~Hz}, 2 \mathrm{H}), 4.88(\mathrm{dd}, J=40.1,13.5 \mathrm{~Hz}, 2 \mathrm{H}), 4.53(\mathrm{t}, J=7.4 \mathrm{~Hz}, 2 \mathrm{H}), 4.13(\mathrm{~d}, J=12.3 \mathrm{~Hz}$, $1 \mathrm{H}), 4.03(\mathrm{t}, J=11.2 \mathrm{~Hz}, 1 \mathrm{H}), 3.77(\mathrm{t}, J=10.6 \mathrm{~Hz}, 1 \mathrm{H}), 3.21-3.10(\mathrm{~m}, 2 \mathrm{H}), 2.89(\mathrm{bs}, 1 \mathrm{H}), 2.17-$ $2.07(\mathrm{~m}, 2 \mathrm{H}), 2.03-1.88(\mathrm{~m}, 2 \mathrm{H}), 1.19(\mathrm{dd}, J=15.4,6.3 \mathrm{~Hz}, 6 \mathrm{H}), 1.06(\mathrm{dd}, J=26.4,6.1 \mathrm{~Hz}, 7 \mathrm{H})$. $\delta 164.3,150.1,147.6,145.9,145.7,144.8,138.0,130.4,129.8,129.4,128.3,127.0,124.3,123.3$, $123.1,119.9,118.0,115.5,65.5,64.0,59.6,58.5,56.0,36.7,28.2,28.1,25.3,24.8,24.0,23.6$, 23.3, 21.2.

Calc. for $\mathrm{C}_{33} \mathrm{H}_{42} \mathrm{~N}_{3} \mathrm{O}_{2}[\mathrm{M}]^{+}:$512.3277, found: 512.3273 .

\section{Compound 26}

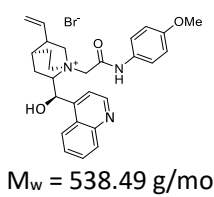

Following Procedure B and using $\mathbf{5}(0.8 \mathrm{~g}, 3.4 \mathrm{mmol})$, the product $\mathbf{2 6}(1.8 \mathrm{~g}, 3.3 \mathrm{mmol}, \mathbf{9 6 \%})$ was obtained as colorless solid $\left(\mathrm{mp} 143-144^{\circ} \mathrm{C} ;[\alpha]_{\mathrm{D}}^{20}=-72.9(c=1.0, \mathrm{MeOH})\right)$.

${ }^{13} \mathrm{C}$ NMR (100 MHz, DMSO-d $)$

HRMS ESI $(\mathrm{m} / \mathrm{z})$

$\delta 11.02(\mathrm{~s}, 1 \mathrm{H}), 8.96(\mathrm{~d}, J=4.3 \mathrm{~Hz}, 1 \mathrm{H}), 8.17(\mathrm{~d}, J=8.3 \mathrm{~Hz}, 1 \mathrm{H}), 8.07(\mathrm{~d}, J=8.3 \mathrm{~Hz}, 1 \mathrm{H}), 7.82-$ $7.75(\mathrm{~m}, 2 \mathrm{H}), 7.71(\mathrm{~d}, J=8.8 \mathrm{~Hz}, 2 \mathrm{H}), 7.55(\mathrm{t}, J=7.5 \mathrm{~Hz}, 1 \mathrm{H}), 7.01(\mathrm{~d}, J=8.8 \mathrm{~Hz}, 2 \mathrm{H}), 6.74(\mathrm{~d}, J=$ $3.2 \mathrm{~Hz}, 1 \mathrm{H}), 6.14(\mathrm{~s}, 1 \mathrm{H}), 5.75-5.63(\mathrm{~m}, 1 \mathrm{H}), 5.20(\mathrm{~d}, J=17.4 \mathrm{~Hz}, 1 \mathrm{H}), 4.98-4.87(\mathrm{~m}, 2 \mathrm{H}), 4.73$ $(\mathrm{d}, J=15.6 \mathrm{~Hz}, 1 \mathrm{H}), 4.51-4.33(\mathrm{~m}, 2 \mathrm{H}), 4.27(\mathrm{t}, J=8.5 \mathrm{~Hz}, 1 \mathrm{H}), 3.97(\mathrm{t}, J=11.4 \mathrm{~Hz}, 1 \mathrm{H}), 3.84-$ $3.69(\mathrm{~m}, 4 \mathrm{H}), 2.87(\mathrm{~s}, 1 \mathrm{H}), 2.07(\mathrm{dd}, J=42.9,16.9 \mathrm{~Hz}, 4 \mathrm{H}), 1.17(\mathrm{t}, J=9.5 \mathrm{~Hz}, 1 \mathrm{H})$.

$\delta 162.1,156.2,150.1,147.5,144.8,138.1,130.8,129.9,129.4,127.1,124.1,122.9,121.3,120.1$, $115.9,114.2,65.0,64.7,60.6,59.4,55.5,55.3,36.9,25.3,24.7,20.8$.

Calc. for $\mathrm{C}_{28} \mathrm{H}_{32} \mathrm{~N}_{3} \mathrm{O}_{3}[\mathrm{M}]^{+}:$: 458.2444, found: 458.2435 .

\section{Compound 27}

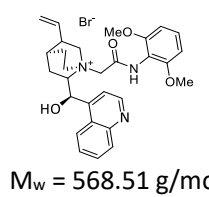

${ }^{1} \mathrm{H}$ NMR $\left(400 \mathrm{MHz}, \mathrm{DMSO}-d_{6}\right)$

${ }^{13} \mathrm{C}$ NMR $\left(100 \mathrm{MHz}, \mathrm{DMSO}-d_{6}\right)$

HRMS ESI $(m / z)$
Following Procedure B and using $6(0.9 \mathrm{~g}, 3.4 \mathrm{mmol})$, the product $27(1.8 \mathrm{~g}, 3.2 \mathrm{mmol}, \mathbf{9 4 \%})$ was obtained as pale brown solid $\left(\mathrm{mp} 159-160^{\circ} \mathrm{C} ;[\alpha]_{\mathrm{D}}^{20}=-27.4(c=1.0, \mathrm{MeOH})\right)$

$\delta 10.01(\mathrm{~s}, 1 \mathrm{H}), 8.97(\mathrm{~d}, J=4.5 \mathrm{~Hz}, 1 \mathrm{H}), 8.17(\mathrm{~d}, J=8.4 \mathrm{~Hz}, 1 \mathrm{H}), 8.06(\mathrm{~d}, J=8.5 \mathrm{~Hz}, 1 \mathrm{H}), 7.82-$ $7.73(\mathrm{~m}, 2 \mathrm{H}), 7.44(\mathrm{t}, J=7.6 \mathrm{~Hz}, 1 \mathrm{H}), 7.31(\mathrm{t}, J=8.4 \mathrm{~Hz}, 1 \mathrm{H}), 6.92(\mathrm{~d}, J=3.2 \mathrm{~Hz}, 1 \mathrm{H}), 6.74(\mathrm{~d}, J=$ $8.4 \mathrm{~Hz}, 2 \mathrm{H}), 6.17(\mathrm{~s}, 1 \mathrm{H}), 5.70-5.58(\mathrm{~m}, 1 \mathrm{H}), 5.17(\mathrm{~d}, J=17.4 \mathrm{~Hz}, 1 \mathrm{H}), 4.91(\mathrm{~d}, J=10.5 \mathrm{~Hz}, 1 \mathrm{H})$, $4.77(\mathrm{~d}, J=16.0 \mathrm{~Hz}, 1 \mathrm{H}), 4.68(\mathrm{~d}, J=16.0 \mathrm{~Hz}, 1 \mathrm{H}), 4.51-4.43(\mathrm{~m}, 1 \mathrm{H}), 4.42-4.34(\mathrm{~m}, 2 \mathrm{H}), 3.90$ $(\mathrm{t}, J=11.4 \mathrm{~Hz}, 1 \mathrm{H}), 3.73-3.66(\mathrm{~m}, 1 \mathrm{H}), 3.59(\mathrm{~s}, 6 \mathrm{H}), 2.87(\mathrm{~s}, 1 \mathrm{H}), 2.14-2.07(\mathrm{~m}, 2 \mathrm{H}), 2.02-1.89$ $(\mathrm{m}, 2 \mathrm{H}), 1.00(\mathrm{t}, \mathrm{J}=10.3 \mathrm{~Hz}, 1 \mathrm{H})$.

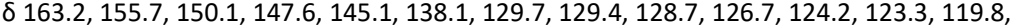
$115.6,112.4,104.4,65.6,63.8,60.3,58.7,56.0,55.8,36.8,25.2,24.7,21.1$. Calc. for $\mathrm{C}_{29} \mathrm{H}_{34} \mathrm{~N}_{3} \mathrm{O}_{4}[\mathrm{M}]^{+}:$: 488.2549, found: 488.2542 . 


\section{Compound 28}

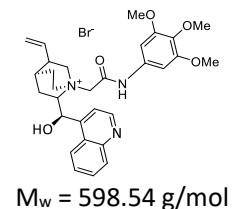

${ }^{1} \mathrm{H}$ NMR $\left(400 \mathrm{MHz}, \mathrm{DMSO}-d_{6}\right)$

${ }^{13} \mathrm{C}$ NMR (100 MHz, DMSO- $\left.d_{6}\right)$

HRMS ESI $(\mathrm{m} / \mathrm{z})$
Following Procedure B and using 12 (1.0 g, $3.4 \mathrm{mmol})$, the product 28 (1.9 g, $3.2 \mathrm{mmol}, \mathbf{9 5 \%})$ was obtained as colorless solid $\left(\mathrm{mp} 147-148^{\circ} \mathrm{C} ;[\alpha]_{\mathrm{D}}^{20}=-73.7(c=1.0, \mathrm{MeOH})\right)$ $\delta 11.07(\mathrm{~s}, 1 \mathrm{H}), 8.97(\mathrm{~d}, J=4.1 \mathrm{~Hz}, 1 \mathrm{H}), 8.21(\mathrm{~d}, J=8.3 \mathrm{~Hz}, 1 \mathrm{H}), 8.07(\mathrm{~d}, J=8.3 \mathrm{~Hz}, 1 \mathrm{H}), 7.84-$ $7.75(\mathrm{~m}, 2 \mathrm{H}), 7.54(\mathrm{t}, J=7.4 \mathrm{~Hz}, 1 \mathrm{H}), 7.16(\mathrm{~s}, 2 \mathrm{H}), 6.75(\mathrm{~d}, J=2.7 \mathrm{~Hz}, 1 \mathrm{H}), 6.16(\mathrm{~s}, 1 \mathrm{H}), 5.79-5.64$ $(\mathrm{m}, 1 \mathrm{H}), 5.19(\mathrm{~d}, J=17.3 \mathrm{~Hz}, 1 \mathrm{H}), 4.93(\mathrm{t}, J=12.7 \mathrm{~Hz}, 2 \mathrm{H}), 4.72(\mathrm{~d}, J=15.6 \mathrm{~Hz}, 1 \mathrm{H}), 4.41(\mathrm{~s}, 1 \mathrm{H})$ $4.27(\mathrm{t}, J=11.3 \mathrm{~Hz}, 2 \mathrm{H}), 4.01(\mathrm{t}, J=11.4 \mathrm{~Hz}, 1 \mathrm{H}), 3.84(\mathrm{~s}, 1 \mathrm{H}), 3.80(\mathrm{~s}, 6 \mathrm{H}), 3.66(\mathrm{~s}, 3 \mathrm{H}), 2.87(\mathrm{~s}$, $1 \mathrm{H}), 2.21-1.98(\mathrm{~m}, 4 \mathrm{H}), 1.20(\mathrm{t}, J=9.7 \mathrm{~Hz}, 1 \mathrm{H})$.

$\delta 162.4,152.9,150.1,147.6,144.8,138.1,134.5,133.8,129.9,129.4,126.9,124.2,123.0,120.0$, $116.1,97.6,65.1,65.0,60.8,60.2,59.5,55.9,55.4,37.0,25.3,24.7,20.7$.

Calc. for $\mathrm{C}_{30} \mathrm{H}_{36} \mathrm{~N}_{3} \mathrm{O}_{5}[\mathrm{M}]^{+}$: 518.2655, found: 518.2646.

\section{Compound 29}

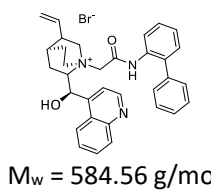

${ }^{1} \mathrm{H}$ NMR $\left(400 \mathrm{MHz}, \mathrm{DMSO}-d_{6}\right)$

${ }^{13} \mathrm{C}$ NMR $\left(100 \mathrm{MHz}, \mathrm{DMSO}-d_{6}\right)$

HRMS ESI $(\mathrm{m} / \mathrm{z})$
Following Procedure B and using 8 (1.0 g, $3.4 \mathrm{mmol})$, the product $29(1.9 \mathrm{~g}, 3.2 \mathrm{mmol}, \mathbf{9 4 \%})$ was obtained as colorless solid (mp $\left.149-150{ }^{\circ} \mathrm{C} ;[\alpha]_{\mathrm{D}}^{20}=-44.6(c=1.1, \mathrm{MeOH})\right)$.

\section{Compound $\mathbf{3 0}$}

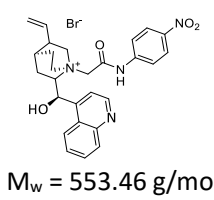

${ }^{1} \mathrm{H}$ NMR $\left(400 \mathrm{MHz}, \mathrm{DMSO}-d_{6}\right)$

${ }^{13} \mathrm{C}$ NMR $\left(100 \mathrm{MHz}\right.$, DMSO- $\left.d_{6}\right)$

HRMS ESI $(\mathrm{m} / \mathrm{z})$
Following Procedure $\mathbf{B}$ and using $\mathbf{9}(0.9 \mathrm{~g}, 3.4 \mathrm{mmol})$, the product $\mathbf{3 0}(1.8 \mathrm{~g}, 3.3 \mathrm{mmol}, \mathbf{9 8 \%})$ was obtained as yellowish solid (mp 235-236 ${ }^{\circ} \mathrm{C}$ (decomposition); $[\alpha]_{\mathrm{D}}^{20}=-82.7(c=1.0$ $\mathrm{MeOH})$ ).

$10.49(\mathrm{~s}, 1 \mathrm{H}), 8.96(\mathrm{~d}, J=4.5 \mathrm{~Hz}, 1 \mathrm{H}), 8.07(\mathrm{dd}, J=13.4,4.8 \mathrm{~Hz}, 2 \mathrm{H}), 7.81-7.76(\mathrm{~m}, 1 \mathrm{H}), 7.74$ $(\mathrm{d}, J=4.5 \mathrm{~Hz}, 1 \mathrm{H}), 7.60-7.54(\mathrm{~m}, 2 \mathrm{H}), 7.52-7.42(\mathrm{~m}, 5 \mathrm{H}), 7.35(\mathrm{t}, J=7.7 \mathrm{~Hz}, 2 \mathrm{H}), 7.22(\mathrm{t}, J=7.4$ $\mathrm{Hz}, 1 \mathrm{H}), 6.70(\mathrm{~d}, J=3.8 \mathrm{~Hz}, 1 \mathrm{H}), 5.93(\mathrm{bs}, 1 \mathrm{H}), 5.67-5.56(\mathrm{~m}, 1 \mathrm{H}), 5.10(\mathrm{~d}, J=17.3 \mathrm{~Hz}, 1 \mathrm{H}), 4.92$ $(\mathrm{d}, J=10.5 \mathrm{~Hz}, 1 \mathrm{H}), 4.74(\mathrm{~d}, J=15.7 \mathrm{~Hz}, 1 \mathrm{H}), 4.48(\mathrm{~d}, J=15.8 \mathrm{~Hz}, 1 \mathrm{H}), 4.34(\mathrm{dd}, J=17.0,8.7 \mathrm{~Hz}$, $1 \mathrm{H}), 4.23(\mathrm{t}, J=9.0 \mathrm{~Hz}, 1 \mathrm{H}), 3.93-3.85(\mathrm{~m}, 1 \mathrm{H}), 3.69-3.58(\mathrm{~m}, 2 \mathrm{H}), 2.77(\mathrm{bs}, 1 \mathrm{H}), 2.11-2.01$ $(\mathrm{m}, 2 \mathrm{H}), 1.97-1.87(\mathrm{~m}, 2 \mathrm{H}), 1.04(\mathrm{t}, J=11.1 \mathrm{~Hz}, 1 \mathrm{H})$.

$\delta 163.1,150.1,147.5,144.8,138.7,137.9,137.8,133.0,130.6,129.8,129.4,128.6,128.5,128.1$ $127.4,127.2,127.0,124.2,123.2,123.2,119.9,115.8,65.3,64.7,60.1,59.0,55.4,36.8,25.2$, 24.6, 20.8.

Calc. for $\mathrm{C}_{33} \mathrm{H}_{34} \mathrm{~N}_{3} \mathrm{O}_{2}[\mathrm{M}]^{+}:$504.2651, found: 504.2644 $\delta 11.67(\mathrm{~s}, 1 \mathrm{H}), 8.97(\mathrm{~d}, J=4.5 \mathrm{~Hz}, 1 \mathrm{H}), 8.40-8.34(\mathrm{~m}, 2 \mathrm{H}), 8.13(\mathrm{~d}, J=8.3 \mathrm{~Hz}, 1 \mathrm{H}), 8.10-8.02$ $(\mathrm{m}, 3 \mathrm{H}), 7.83-7.75(\mathrm{~m}, 2 \mathrm{H}), 7.63(\mathrm{ddd}, J=8.3,6.9,1.2 \mathrm{~Hz}, 1 \mathrm{H}), 6.76(\mathrm{~d}, J=3.7 \mathrm{~Hz}, 1 \mathrm{H}), 6.14(\mathrm{~s}$ $1 \mathrm{H}), 5.77-5.67(\mathrm{~m}, 1 \mathrm{H}), 5.21(\mathrm{~d}, J=17.3 \mathrm{~Hz}, 1 \mathrm{H}), 5.03-4.93(\mathrm{~m}, 2 \mathrm{H}), 4.81(\mathrm{~d}, J=16.1 \mathrm{~Hz}, 1 \mathrm{H})$ $4.50-4.41(\mathrm{~m}, 1 \mathrm{H}), 4.34-4.26(\mathrm{~m}, 2 \mathrm{H}), 4.00(\mathrm{dd}, J=12.6,10.4 \mathrm{~Hz}, 1 \mathrm{H}), 3.85-3.76(\mathrm{~m}, 1 \mathrm{H}), 2.88$ (s, $1 \mathrm{H}), 2.17-1.97(\mathrm{~m}, 4 \mathrm{H}), 1.20(\mathrm{t}, J=11.0 \mathrm{~Hz}, 1 \mathrm{H})$ $\delta 163.7,150.1,147.5,144.7,143.7,143.2,138.1,129.9,129.4,127.1,125.2,124.1,122.8,120.0$ $119.6,115.9,65.1,64.9,60.6,59.4,55.6,36.9,25.2,24.7,20.7$ Calc. for $\mathrm{C}_{27} \mathrm{H}_{29} \mathrm{~N}_{4} \mathrm{O}_{4}[\mathrm{M}]^{+}:$473.2189, found: 473.2186 


\section{Compound 31}

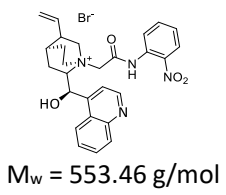

${ }^{1} \mathrm{H}$ NMR $\left(400 \mathrm{MHz}, \mathrm{DMSO}-d_{6}\right)$

${ }^{13} \mathrm{C}$ NMR $\left(100 \mathrm{MHz}, \mathrm{DMSO}-d_{6}\right)$

HRMS ESI (m/z)
Following Procedure $\mathbf{B}$ and using 10 (0.9 g, $3.4 \mathrm{mmol})$, the product 31 (1.8 g, $3.3 \mathrm{mmol}, \mathbf{9 7 \%})$ was obtained as yellowish solid (mp $165-167{ }^{\circ} \mathrm{C}$ (decomposition); $[\alpha]_{\mathrm{D}}^{20}=-23.9(c=1.0$, $\mathrm{MeOH})$ ).

$\delta 11.39(\mathrm{~s}, 1 \mathrm{H}), 8.97(\mathrm{~d}, J=4.5 \mathrm{~Hz}, 1 \mathrm{H}), 8.15-8.05(\mathrm{~m}, 3 \mathrm{H}), 7.89-7.82(\mathrm{~m}, 1 \mathrm{H}), 7.81-7.75(\mathrm{~m}$ $2 \mathrm{H}), 7.70(\mathrm{~d}, J=7.2 \mathrm{~Hz}, 1 \mathrm{H}), 7.59-7.51(\mathrm{~m}, 2 \mathrm{H}), 6.83(\mathrm{~d}, J=3.7 \mathrm{~Hz}, 1 \mathrm{H}), 6.13(\mathrm{~s}, 1 \mathrm{H}), 5.71-5.62$ $(\mathrm{m}, 1 \mathrm{H}), 5.16(\mathrm{~d}, J=17.4 \mathrm{~Hz}, 1 \mathrm{H}), 4.98-4.88(\mathrm{~m}, 2 \mathrm{H}), 4.73(\mathrm{~d}, J=16.1 \mathrm{~Hz}, 1 \mathrm{H}), 4.50-4.36(\mathrm{~m}$, $1 \mathrm{H}), 4.29(\mathrm{t}, J=9.2 \mathrm{~Hz}, 1 \mathrm{H}), 4.11(\mathrm{~d}, J=12.5 \mathrm{~Hz}, 1 \mathrm{H}), 4.00-3.91(\mathrm{~m}, 1 \mathrm{H}), 3.74(\mathrm{t}, J=10.6 \mathrm{~Hz}, 1 \mathrm{H})$, 2.87 (bs, $1 \mathrm{H}$ ), $2.16-2.06(\mathrm{~m}, 2 \mathrm{H}), 2.03-1.93(\mathrm{~m}, 2 \mathrm{H}), 1.09(\mathrm{t}, J=11.1 \mathrm{~Hz}, 1 \mathrm{H})$. $\delta 163.3,150.1,147.5,144.8,143.4,137.9,134.3,129.7,129.4,129.0,127.0(x 2), 126.3,125.2$, $124.2,123.3,120.0,115.8,65.2,64.9,60.3,59.0,55.7,36.8,25.2,24.7,21.0$ Calc. for $\mathrm{C}_{27} \mathrm{H}_{29} \mathrm{~N}_{4} \mathrm{O}_{4}[\mathrm{M}]^{+}:$473.2189, found: 473.2177 .

\section{Compound 32}

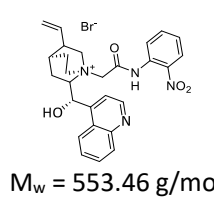

${ }^{1} \mathrm{H}$ NMR $\left(400 \mathrm{MHz}, \mathrm{DMSO}-d_{6}\right)$

${ }^{13} \mathrm{C}$ NMR (100 MHz, DMSO- $\left.d_{6}\right)$

HRMS ESI $(\mathrm{m} / \mathrm{z})$
Following Procedure B, using cinchonine instead of cinchonidine and using $\mathbf{3 2}(0.9 \mathrm{~g}, 3.4 \mathrm{mmol})$, the product $10(1.8 \mathrm{~g}, 3.3 \mathrm{mmol}, \mathbf{9 5 \%})$ was obtained as yellowish solid (mp $164-165^{\circ} \mathrm{C} ;[\alpha]_{\mathrm{D}}^{20}=$ $38.6(c=1.0, \mathrm{MeOH})$.

$\delta 11.71(\mathrm{~s}, 1 \mathrm{H}), 8.96(\mathrm{~d}, J=4.4 \mathrm{~Hz}, 1 \mathrm{H}), 8.12(\mathrm{~d}, J=8.3 \mathrm{~Hz}, 1 \mathrm{H}), 8.07(\mathrm{dd}, J=8.2,2.6 \mathrm{~Hz}, 2 \mathrm{H}), 7.83$ $-7.68(\mathrm{~m}, 4 \mathrm{H}), 7.51(\mathrm{dt}, J=21.7,7.6 \mathrm{~Hz}, 2 \mathrm{H}), 7.04(\mathrm{~s}, 1 \mathrm{H}), 6.14(\mathrm{~s}, 1 \mathrm{H}), 6.06-5.92(\mathrm{~m}, 1 \mathrm{H}), 5.31$ $-5.19(\mathrm{~m}, 2 \mathrm{H}), 5.01(\mathrm{~d}, J=14.4 \mathrm{~Hz}, 1 \mathrm{H}), 4.73(\mathrm{~d}, J=13.6 \mathrm{~Hz}, 1 \mathrm{H}), 4.43(\mathrm{t}, J=9.0 \mathrm{~Hz}, 1 \mathrm{H}), 4.25-$ $4.13(\mathrm{~m}, 2 \mathrm{H}), 3.94-3.84(\mathrm{~m}, 1 \mathrm{H}), 3.71(\mathrm{~d}, J=8.0 \mathrm{~Hz}, 1 \mathrm{H}), 2.84(\mathrm{~d}, J=6.3 \mathrm{~Hz}, 1 \mathrm{H}), 2.16(\mathrm{t}, J=11.8$ $\mathrm{Hz}, 1 \mathrm{H}), 1.92(\mathrm{~s}, 3 \mathrm{H}), 0.97(\mathrm{t}, J=7.4 \mathrm{~Hz}, 1 \mathrm{H})$

$\delta 163.4,150.1,147.6,144.7,143.5,136.5,134.2,129.7,129.3,129.1,126.9$ (x2), 126.3, 125.1 , $124.4,123.4,120.0,117.1,65.4,65.1,59.1,58.8,57.0,37.1,26.1,22.9,20.6$. Calc. for $\mathrm{C}_{27} \mathrm{H}_{29} \mathrm{~N}_{4} \mathrm{O}_{4}[\mathrm{M}]^{+}:$473.2189, found: 473.2187 .

\section{Compound 33}

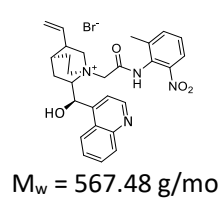

${ }^{1} \mathrm{H}$ NMR (400 MHz, DMSO- $\left.d_{6}\right)$

${ }^{13} \mathrm{C}$ NMR (100 MHz, DMSO- $\left.d_{6}\right)$

HRMS ESI $(\mathrm{m} / \mathrm{z})$
Following Procedure B and using 11 (0.9 g, $3.4 \mathrm{mmol})$, the product $\mathbf{3 3}$ (1.8 g, $3.2 \mathrm{mmol}, \mathbf{9 5 \%})$ was obtained as colorless solid (mp $217-218{ }^{\circ} \mathrm{C}$ (decomposition); $[\alpha]_{\mathrm{D}}^{20}=-56.5(c=1.1$, $\mathrm{MeOH})$ ).

$\delta 10.68(\mathrm{~s}, 1 \mathrm{H}), 8.97(\mathrm{~d}, J=4.5 \mathrm{~Hz}, 1 \mathrm{H}), 8.23-8.15(\mathrm{~m}, 3 \mathrm{H}), 8.07(\mathrm{dd}, J=9.2,2.8 \mathrm{~Hz}, 2 \mathrm{H}), 7.81-$ $7.74(\mathrm{~m}, 2 \mathrm{H}), 7.56(\mathrm{t}, J=7.3 \mathrm{~Hz}, 1 \mathrm{H}), 6.78(\mathrm{~d}, J=3.9 \mathrm{~Hz}, 1 \mathrm{H}), 6.19(\mathrm{~s}, 1 \mathrm{H}), 5.76-5.63(\mathrm{~m}, 1 \mathrm{H})$, $5.20(\mathrm{~d}, J=17.7 \mathrm{~Hz}, 2 \mathrm{H}), 4.91(\mathrm{dd}, J=19.3,13.3 \mathrm{~Hz}, 2 \mathrm{H}), 4.54-4.42(\mathrm{~m}, 1 \mathrm{H}), 4.30(\mathrm{~d}, J=10.1 \mathrm{~Hz}$ $2 \mathrm{H}), 4.05(\mathrm{t}, J=11.5 \mathrm{~Hz}, 1 \mathrm{H}), 3.84(\mathrm{t}, J=10.2 \mathrm{~Hz}, 1 \mathrm{H}), 2.88(\mathrm{~s}, 1 \mathrm{H}), 2.51(\mathrm{~s}, 3 \mathrm{H}), 2.19-1.99(\mathrm{~m}$ $4 \mathrm{H}), 1.17(\mathrm{t}, J=11.5 \mathrm{~Hz}, 1 \mathrm{H})$.

$\delta 163.7,150.1,147.5,144.8,144.3,141.1,138.0,132.6,129.9,129.4,127.0,125.6,124.5,124.2$, 123.0, 121.8, 120.0, 115.9, 65.1, 64.9, 60.5, 59.3, 55.5, 36.9, 25.3, 24.7, 20.9, 18.3.

Calc. for $\mathrm{C}_{28} \mathrm{H}_{31} \mathrm{~N}_{4} \mathrm{O}_{4}[\mathrm{M}]^{+}:$: 487.2345, found: 487.2338 . 


\section{Compound 34}

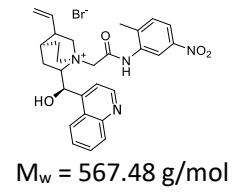

${ }^{1} \mathrm{H}$ NMR $\left(400 \mathrm{MHz}, \mathrm{DMSO}-d_{6}\right)$

${ }^{13} \mathrm{C}$ NMR (100 MHz, DMSO- $\left.d_{6}\right)$

HRMS ESI $(\mathrm{m} / \mathrm{z})$
Following Procedure B and using 12 (0.9 g, $3.4 \mathrm{mmol})$, the product 34 (1.9 g, $3.3 \mathrm{mmol}, \mathbf{9 6 \%})$ was obtained as colorless solid (mp 206-207 ${ }^{\circ} \mathrm{C}$ (decomposition); $[\alpha]_{\mathrm{D}}^{20}=-38.3(c=1.0$, $\mathrm{MeOH})$ ).

$\delta 10.68(\mathrm{~s}, 1 \mathrm{H}), 8.97(\mathrm{~d}, J=4.4 \mathrm{~Hz}, 1 \mathrm{H}), 8.65(\mathrm{~d}, J=1.9 \mathrm{~Hz}, 1 \mathrm{H}), 8.21(\mathrm{~d}, J=8.5 \mathrm{~Hz}, 1 \mathrm{H}), 8.07(\mathrm{~d}, J$ $=8.2 \mathrm{~Hz}, 2 \mathrm{H}), 7.85-7.74(\mathrm{~m}, 2 \mathrm{H}), 7.61(\mathrm{~d}, J=8.5 \mathrm{~Hz}, 1 \mathrm{H}), 7.54(\mathrm{t}, J=7.5 \mathrm{~Hz}, 1 \mathrm{H}), 6.78(\mathrm{~d}, J=3.2$ $\mathrm{Hz}, 1 \mathrm{H}), 6.21(\mathrm{~s}, 1 \mathrm{H}), 5.76-5.65(\mathrm{~m}, 1 \mathrm{H}), 5.16(\mathrm{dd}, J=27.0,16.7 \mathrm{~Hz}, 2 \mathrm{H}), 4.89(\mathrm{dd}, J=37.5,13.3$ $\mathrm{Hz}, 2 \mathrm{H}), 4.54-4.42(\mathrm{~m}, 1 \mathrm{H}), 4.39-4.25(\mathrm{~m}, 2 \mathrm{H}), 4.04(\mathrm{t}, J=11.4 \mathrm{~Hz}, 1 \mathrm{H}), 3.83(\mathrm{t}, J=10.6 \mathrm{~Hz}$, $1 \mathrm{H}), 2.88(\mathrm{~s}, 1 \mathrm{H}), 2.50(\mathrm{~s}, 3 \mathrm{H}), 2.19-2.06(\mathrm{~m}, 4 \mathrm{H}), 1.19(\mathrm{t}, J=10.8 \mathrm{~Hz}, 1 \mathrm{H})$.

$\delta 163.7,150.1,147.6,145.7,144.8,139.8,138.1,135.7,131.7,129.9,129.4,126.9,124.2,123.1$, $120.5,120.1,119.2,115.9,65.1,64.8,60.6,59.2,55.6,36.9,25.3,24.7,20.8,18.5$.

Calc. for $\mathrm{C}_{28} \mathrm{H}_{31} \mathrm{~N}_{4} \mathrm{O}_{4}[\mathrm{M}]^{+}:$: 487.2345, found: 487.2340 .

\section{Compound 35}

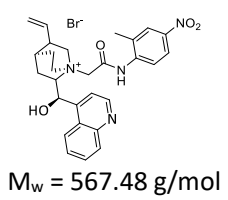

${ }^{1} \mathbf{H}$ NMR $\left(400 \mathrm{MHz}, \mathrm{DMSO}-d_{6}\right)$

${ }^{13} \mathrm{C}$ NMR (100 MHz, DMSO- $\left.d_{6}\right)$

HRMS ESI $(\mathrm{m} / \mathrm{z})$
Following Procedure B and using 13 (0.9 g, $3.4 \mathrm{mmol})$, the product 35 (1.9 g, $3.3 \mathrm{mmol}, \mathbf{9 8 \%})$ was obtained as colorless solid $\left(\mathrm{mp} 161-162{ }^{\circ} \mathrm{C} ;[\alpha]_{\mathrm{D}}^{20}=-24.7(c=1.0, \mathrm{MeOH})\right)$.

$\delta 11.04(\mathrm{~s}, 1 \mathrm{H}), 8.97(\mathrm{~d}, J=3.6 \mathrm{~Hz}, 1 \mathrm{H}), 8.09$ (dd, $J=24.7,8.2 \mathrm{~Hz}, 2 \mathrm{H}), 7.90(\mathrm{~d}, J=7.8 \mathrm{~Hz}, 1 \mathrm{H})$ $7.83-7.67(\mathrm{~m}, 3 \mathrm{H}), 7.51(\mathrm{dd}, J=16.5,8.1 \mathrm{~Hz}, 2 \mathrm{H}), 6.91(\mathrm{~d}, J=2.2 \mathrm{~Hz}, 1 \mathrm{H}), 6.12(\mathrm{~s}, 1 \mathrm{H}), 5.71-$ $5.57(\mathrm{~m}, 1 \mathrm{H}), 5.10(\mathrm{dd}, J=40.3,16.7 \mathrm{~Hz}, 2 \mathrm{H}), 4.88(\mathrm{dd}, J=24.6,13.4 \mathrm{~Hz}, 2 \mathrm{H}), 4.49(\mathrm{t}, J=6.2 \mathrm{~Hz}$, $1 \mathrm{H}), 4.34(\mathrm{t}, J=8.5 \mathrm{~Hz}, 1 \mathrm{H}), 4.14(\mathrm{~d}, J=11.3 \mathrm{~Hz}, 1 \mathrm{H}), 4.00(\mathrm{t}, J=11.0 \mathrm{~Hz}, 1 \mathrm{H}), 3.78(\mathrm{t}, J=9.5 \mathrm{~Hz}$, $1 \mathrm{H}), 2.88$ (bs, 1H), 2.45 (s, 3H), 2.09 (bs, $2 \mathrm{H}), 2.03-1.90(\mathrm{~m}, 2 \mathrm{H}), 1.05(\mathrm{t}, J=10.3 \mathrm{~Hz}, 1 \mathrm{H})$. $\delta 163.6,150.1,147.5,146.2,144.8,137.9,137.2,135 ., 129.8,129.4,127.7,126.9$ (x2), 124.2, $123.2,122.6,119.9,115.7,65.3,64.5,60.1,58.6,55.8,36.7,25.2,24.7,21.2,18.1$. Calc. for $\mathrm{C}_{28} \mathrm{H}_{31} \mathrm{~N}_{4} \mathrm{O}_{4}[\mathrm{M}]^{+}:$487.2345, found: 487.2333 .

\section{Compound 36}

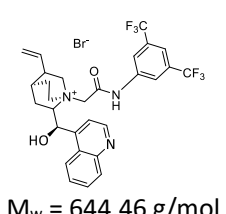

${ }^{1} \mathrm{H}$ NMR (400 MHz, DMSO- $\left.d_{6}\right)$

${ }^{13} \mathrm{C}$ NMR $\left(100 \mathrm{MHz}, \mathrm{DMSO}-d_{6}\right)$

HRMS ESI $(\mathrm{m} / \mathrm{z})$
Following Procedure B and using $14(1.0 \mathrm{~g}, 3.4 \mathrm{mmol})$, the product 36 (1.7 g, $3.2 \mathrm{mmol}, \mathbf{9 3 \%})$ was obtained as colorless solid $\left(\mathrm{mp} 210-211^{\circ} \mathrm{C} ;[\alpha]_{\mathrm{D}}^{20}=-61.5(c=1.0, \mathrm{MeOH})\right)$.

$\delta 11.85(\mathrm{~s}, 1 \mathrm{H}), 8.97(\mathrm{~d}, J=4.5 \mathrm{~Hz}, 1 \mathrm{H}), 8.44(\mathrm{~s}, 2 \mathrm{H}), 8.20(\mathrm{~d}, J=8.4 \mathrm{~Hz}, 1 \mathrm{H}), 8.07$ (dd, $J=8.4,0.8$ $\mathrm{Hz}, 1 \mathrm{H}), 7.94(\mathrm{~s}, 1 \mathrm{H}), 7.83-7.77(\mathrm{~m}, 2 \mathrm{H}), 7.50$ (ddd, $J=8.2,7.0,1.0 \mathrm{~Hz}, 1 \mathrm{H}), 6.74(\mathrm{~d}, J=3.6 \mathrm{~Hz}$, $1 \mathrm{H}), 6.20(\mathrm{~s}, 1 \mathrm{H}), 5.78-5.65(\mathrm{~m}, 1 \mathrm{H}), 5.20(\mathrm{~d}, J=17.3 \mathrm{~Hz}, 1 \mathrm{H}), 4.98(\mathrm{dd}, J=18.0,13.3 \mathrm{~Hz}, 2 \mathrm{H})$, $4.79(\mathrm{~d}, J=15.9 \mathrm{~Hz}, 1 \mathrm{H}), 4.49-4.39(\mathrm{~m}, 1 \mathrm{H}), 4.30(\mathrm{t}, J=8.9 \mathrm{~Hz}, 1 \mathrm{H}), 4.24-4.16(\mathrm{~m}, 1 \mathrm{H}), 4.03$ (dd, $J=12.5,10.5 \mathrm{~Hz}, 1 \mathrm{H}$ ), $3.85(\mathrm{td}, J=11.3,3.2 \mathrm{~Hz}, 1 \mathrm{H}), 2.88(\mathrm{~s}, 1 \mathrm{H}), 2.19-1.96(\mathrm{~m}, 4 \mathrm{H}), 1.23$ (t, $J=10.7 \mathrm{~Hz}, 1 \mathrm{H}$ ).

$\delta 163.8,150.2,147.6,144.8,139.6,138.0,131.2$, 130.8, 129.9, 129.4, 126.6, 124.4, 124.2, 123.1, 121.7, 120.1, 119.6, 119.6, 117.5, 116.1, 65.4, 64.9, 60.8, 59.4, 55.3, 37.0, 25.3, 24.7, 20.7.

Calc. for $\mathrm{C}_{29} \mathrm{H}_{28} \mathrm{~F}_{6} \mathrm{~N}_{3} \mathrm{O}_{2}[\mathrm{M}]^{+}$: 564.2086 , found: 564.2081 . 


\section{Compound 37}

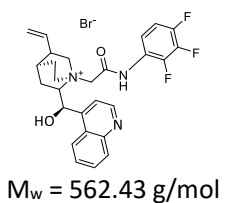

${ }^{1} \mathrm{H}$ NMR $\left(400 \mathrm{MHz}, \mathrm{DMSO}-d_{6}\right)$

${ }^{13} \mathrm{C}$ NMR (100 MHz, DMSO- $\left.d_{6}\right)$

HRMS ESI (m/z)
Following Procedure B and using 15 (0.9 g, $3.4 \mathrm{mmol})$, the product 37 (1.8 g, $3.3 \mathrm{mmol}, \mathbf{9 6 \%})$ was obtained as colorless solid $\left(\mathrm{mp} 207-208^{\circ} \mathrm{C} ;[\alpha]_{\mathrm{D}}^{20}=-56.4(c=1.0, \mathrm{MeOH})\right)$. $\delta 11.02(\mathrm{~s}, 1 \mathrm{H}), 8.97(\mathrm{~d}, J=4.5 \mathrm{~Hz}, 1 \mathrm{H}), 8.14-8.05(\mathrm{~m}, 2 \mathrm{H}), 7.82-7.69(\mathrm{~m}, 3 \mathrm{H}), 7.57$ (ddd, $J=$ $8.3,6.9,1.1 \mathrm{~Hz}, 1 \mathrm{H}), 7.51-7.42(\mathrm{~m}, 1 \mathrm{H}), 6.78(\mathrm{~d}, J=3.8 \mathrm{~Hz}, 1 \mathrm{H}), 6.12(\mathrm{bt}, J=2.4 \mathrm{~Hz}, 1 \mathrm{H}), 5.75-$ $5.64(\mathrm{~m}, 1 \mathrm{H}), 5.17(\mathrm{~d}, J=17.3 \mathrm{~Hz}, 1 \mathrm{H}), 4.96(\mathrm{t}, J=13.7 \mathrm{~Hz}, 2 \mathrm{H}), 4.81(\mathrm{~d}, J=16.2 \mathrm{~Hz}, 1 \mathrm{H}), 4.50-$ $4.39(\mathrm{~m}, 1 \mathrm{H}), 4.33-4.19(\mathrm{~m}, 2 \mathrm{H}), 4.00(\mathrm{dd}, J=12.5,10.5 \mathrm{~Hz}, 1 \mathrm{H}), 3.83-3.70(\mathrm{~m}, 1 \mathrm{H}), 2.88$ (bs, $1 \mathrm{H}), 2.18-1.95(\mathrm{~m}, 4 \mathrm{H}), 1.16(\mathrm{t}, J=11.4 \mathrm{~Hz}, 1 \mathrm{H})$

$\delta 163.6,150.1,147.6,144.7,138.0,129.9,129.4,126.9,124.2,122.9,120.0,119.7,115.9$, 112.4 (x2), 112.2 (x2), 109.5 (x2), 65.1, 64.9, 60.5, 59.0, 55.5, 36.8, 25.2, 24.7, 20.8. Calc. for $\mathrm{C}_{27} \mathrm{H}_{27} \mathrm{~N}_{3} \mathrm{O}_{2} \mathrm{~F}_{3}[\mathrm{M}]^{+}:$: 482.2055, found: 482.2042 .

\section{Compound 38}

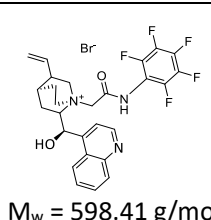

${ }^{1} \mathrm{H}$ NMR $\left(400 \mathrm{MHz}, \mathrm{DMSO}-d_{6}\right)$

${ }^{13} \mathrm{C}$ NMR $\left(100 \mathrm{MHz}\right.$, DMSO- $\left.d_{6}\right)$

HRMS ESI $(\mathrm{m} / \mathrm{z})$
Following Procedure B and using 16 (1.0 g, $3.4 \mathrm{mmol})$, the product $\mathbf{3 8}(1.9 \mathrm{~g}, 3.2 \mathrm{mmol}, \mathbf{9 5 \%})$ was obtained as colorless solid $\left(\mathrm{mp} 201-202^{\circ} \mathrm{C} ;[\alpha]_{\mathrm{D}}^{20}=-44.4(c=1.0, \mathrm{MeOH})\right)$.

$\delta 11.34(\mathrm{~s}, 1 \mathrm{H}), 8.97(\mathrm{~d}, J=4.5 \mathrm{~Hz}, 1 \mathrm{H}), 8.08(\mathrm{~d}, J=8.8 \mathrm{~Hz}, 2 \mathrm{H}), 7.83-7.75(\mathrm{~m}, 2 \mathrm{H}), 7.57(\mathrm{t}, J=$ $8.1 \mathrm{~Hz}, 1 \mathrm{H}), 6.87(\mathrm{~d}, J=3.9 \mathrm{~Hz}, 1 \mathrm{H}), 6.07(\mathrm{~s}, 1 \mathrm{H}), 5.73-5.61(\mathrm{~m}, 1 \mathrm{H}), 5.16(\mathrm{~d}, J=17.4 \mathrm{~Hz}, 1 \mathrm{H})$, $5.09-4.89(\mathrm{~m}, 3 \mathrm{H}), 4.46(\mathrm{t}, J=8.2 \mathrm{~Hz}, 1 \mathrm{H}), 4.30(\mathrm{t}, J=9.2 \mathrm{~Hz}, 1 \mathrm{H}), 4.26-4.19(\mathrm{~m}, 1 \mathrm{H}), 3.99(\mathrm{dd}$ $J=12.3,10.5 \mathrm{~Hz}, 1 \mathrm{H}), 3.79(\mathrm{t}, J=10.5 \mathrm{~Hz}, 1 \mathrm{H}), 2.89(\mathrm{~s}, 1 \mathrm{H}), 2.19-2.07(\mathrm{~m}, 2 \mathrm{H}), 2.06-1.94(\mathrm{~m}$, $2 \mathrm{H}), 1.13(\mathrm{t}, J=11.4 \mathrm{~Hz}, 1 \mathrm{H})$.

$\delta 163.9,150.1,147.6,144.6,138.0,129.9,129.5,126.9,124.1,122.8,120.0,115.8,65.2,64.8$, 60.3, 58.4, 55.7, 36.7, 25.2, 24.6, 20.9.

Calc. for $\mathrm{C}_{27} \mathrm{H}_{25} \mathrm{~N}_{3} \mathrm{O}_{2} \mathrm{~F}_{5}[\mathrm{M}]^{+}:$518.1867, found: 518.1851 .

\section{Compound 39}

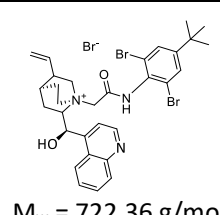

${ }^{1} \mathrm{H}$ NMR $\left(400 \mathrm{MHz}, \mathrm{DMSO}-d_{6}\right)$

${ }^{13} \mathrm{C}$ NMR $\left(100 \mathrm{MHz}, \mathrm{DMSO}-d_{6}\right)$

HRMS ESI $(\mathrm{m} / \mathrm{z})$
Following Procedure B and using 17 (1.5 g, $3.4 \mathrm{mmol})$, the product $39(2.2 \mathrm{~g}, 3.1 \mathrm{mmol}, \mathbf{9 1 \%})$ was obtained as colorless solid $\left(\mathrm{mp} 178-179^{\circ} \mathrm{C} ;[\alpha]_{\mathrm{D}}^{20}=-56.1(c=1.0, \mathrm{MeOH})\right)$.

$\delta 10.98(\mathrm{~s}, 1 \mathrm{H}), 8.97(\mathrm{~d}, J=4.5 \mathrm{~Hz}, 1 \mathrm{H}), 8.12(\mathrm{~d}, J=8.5 \mathrm{~Hz}, 1 \mathrm{H}), 8.05(\mathrm{~d}, J=8.3 \mathrm{~Hz}, 1 \mathrm{H}), 7.79-$ $7.73(\mathrm{~m}, 4 \mathrm{H}), 7.48(\mathrm{t}, J=7.5 \mathrm{~Hz}, 1 \mathrm{H}), 6.94(\mathrm{~d}, J=3.5 \mathrm{~Hz}, 1 \mathrm{H}), 6.15(\mathrm{~s}, 1 \mathrm{H}), 5.70-5.60(\mathrm{~m}, 1 \mathrm{H})$, $5.16(\mathrm{~d}, J=17.4 \mathrm{~Hz}, 1 \mathrm{H}), 4.97-4.89(\mathrm{~m}, 2 \mathrm{H}), 4.81(\mathrm{~d}, J=16.6 \mathrm{~Hz}, 1 \mathrm{H}), 4.55-4.47(\mathrm{~m}, 1 \mathrm{H}), 4.43$ $(\mathrm{t}, J=9.1 \mathrm{~Hz}, 1 \mathrm{H}), 4.34-4.27(\mathrm{~m}, 1 \mathrm{H}), 4.00(\mathrm{t}, J=11.4 \mathrm{~Hz}, 1 \mathrm{H}), 3.80(\mathrm{t}, J=10.5 \mathrm{~Hz}, 1 \mathrm{H}), 2.89(\mathrm{~s}$, $1 \mathrm{H}), 2.17-2.08(\mathrm{~m}, 2 \mathrm{H}), 2.03-1.92(\mathrm{~m}, 2 \mathrm{H}), 1.31(\mathrm{~s}, 9 \mathrm{H}), 1.04(\mathrm{t}, J=9.2 \mathrm{~Hz}, 1 \mathrm{H})$. $\delta 163.7,154.3,150.1,147.6,144.7,138.0,129.8,129.4,128.6,126.8,124.1,123.4,123.3,119.9$, 115.6, 65.6, 63.9, 60.2, 58.3, 56.0, 36.7, 34.8, 30.6, 25.2, 24.7, 21.1 Calc. for $\mathrm{C}_{31} \mathrm{H}_{36} \mathrm{~N}_{3} \mathrm{O}_{2} \mathrm{Br}_{2}[\mathrm{M}]^{+}$: 640.1174, found: 640.1161 . 


\section{Compound $\mathbf{4 0}$}

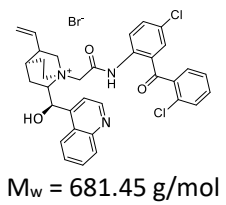

${ }^{1} \mathbf{H}$ NMR $\left(400 \mathrm{MHz}, \mathrm{DMSO}-d_{6}\right)$

${ }^{13} \mathrm{C}$ NMR $\left(100 \mathrm{MHz}, \mathrm{DMSO}-d_{6}\right)$

HRMS ESI $(\mathrm{m} / \mathrm{z})$
Following Procedure B and using 18 (1.3 g, $3.4 \mathrm{mmol})$, the product 40 (2.2 g, $3.2 \mathrm{mmol}$, 93\%) was obtained as colorless solid $\left(\mathrm{mp} 163-164^{\circ} \mathrm{C} ;[\alpha]_{\mathrm{D}}^{20}=-53.8(c=1.0, \mathrm{MeOH})\right)$.

$\delta 11.38(\mathrm{~s}, 1 \mathrm{H}), 8.94(\mathrm{~d}, J=4.5 \mathrm{~Hz}, 1 \mathrm{H}), 8.10(\mathrm{~d}, J=8.5 \mathrm{~Hz}, 1 \mathrm{H}), 8.05(\mathrm{~d}, J=8.4 \mathrm{~Hz}, 1 \mathrm{H}), 7.90-$ $7.82(\mathrm{~m}, 2 \mathrm{H}), 7.77-7.73(\mathrm{~m}, 2 \mathrm{H}), 7.67-7.60(\mathrm{~m}, 3 \mathrm{H}), 7.56-7.51(\mathrm{~m}, 1 \mathrm{H}), 7.45-7.38(\mathrm{~m}, 2 \mathrm{H})$, $6.76(\mathrm{~d}, J=3.9 \mathrm{~Hz}, 1 \mathrm{H}), 6.12(\mathrm{~s}, 1 \mathrm{H}), 5.68-5.56(\mathrm{~m}, 1 \mathrm{H}), 5.10(\mathrm{~d}, J=17.3 \mathrm{~Hz}, 1 \mathrm{H}), 4.96(\mathrm{~d}, J=16.0$ $\mathrm{Hz}, 1 \mathrm{H}), 4.85(\mathrm{~d}, J=10.5 \mathrm{~Hz}, 1 \mathrm{H}), 4.58(\mathrm{~d}, J=16.0 \mathrm{~Hz}, 1 \mathrm{H}), 4.47-4.36(\mathrm{~m}, 1 \mathrm{H}), 4.24(\mathrm{t}, J=9.2 \mathrm{~Hz}$, $1 \mathrm{H}), 4.08(\mathrm{~d}, J=12.5 \mathrm{~Hz}, 1 \mathrm{H}$ ), $3.84(\mathrm{t}, J=11.5 \mathrm{~Hz}, 1 \mathrm{H}), 3.71(\mathrm{t}, J=11.1 \mathrm{~Hz}, 1 \mathrm{H}), 2.83(\mathrm{~s}, 1 \mathrm{H}), 2.15$ $-2.05(\mathrm{~m}, 2 \mathrm{H}), 2.00-1.92(\mathrm{~m}, 2 \mathrm{H}), 1.06(\mathrm{t}, J=11.3 \mathrm{~Hz}, 1 \mathrm{H})$

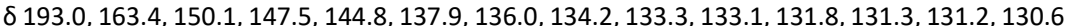
$130.4,129.8,129.7,129.3,127.3,126.8,126.3,124.2,123.0,120.0,115.7,65.1,64.7,60.1,59.2$, $55.6,36.7,25.2,24.7,20.9$.

Calc. for $\mathrm{C}_{34} \mathrm{H}_{32} \mathrm{~N}_{3} \mathrm{O}_{3}[\mathrm{M}]^{+}: 600.1821$, found: 600.1805 .

\section{Compound 41}

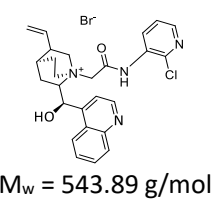

${ }^{1} \mathrm{H}$ NMR (400 MHz, DMSO-d $)$

${ }^{13} \mathrm{C}$ NMR (100 MHz, DMSO-d $)$

HRMS ESI $(m / z)$
Following Procedure B and using 19 (0.8 g, $3.4 \mathrm{mmol})$, the product $41(1.7 \mathrm{~g}, 3.2 \mathrm{mmol}, \mathbf{9 4 \%})$ was obtained as colorless solid $\left(\mathrm{mp} 152-153^{\circ} \mathrm{C} ;[\alpha]_{\mathrm{D}}^{20}=-53.5(c=1.0, \mathrm{MeOH})\right)$

\section{Compound 42}

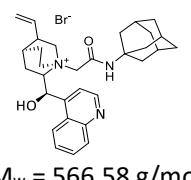

${ }^{1} \mathbf{H}$ NMR (400 MHz, DMSO-d $)$

${ }^{13} \mathrm{C}$ NMR $\left(100 \mathrm{MHz}\right.$, DMSO- $\left.d_{6}\right)$

HRMS ESI $(\mathrm{m} / \mathrm{z})$
Following Procedure B and using 20 (0.9 g, $3.4 \mathrm{mmol})$, the product 42 (1.9 g, $3.3 \mathrm{mmol}, \mathbf{9 7 \%})$ was obtained as colorless solid (mp $257-258{ }^{\circ} \mathrm{C}$ (decomposition); $[\alpha]_{\mathrm{D}}^{20}=-69.9(c=1.0$, $\mathrm{MeOH})$ ).

$\delta 10.85(\mathrm{~s}, 1 \mathrm{H}), 8.97(\mathrm{~d}, J=4.5 \mathrm{~Hz}, 1 \mathrm{H}), 8.36(\mathrm{dd}, J=4.6,1.6 \mathrm{~Hz}, 1 \mathrm{H}), 8.29(\mathrm{dd}, J=8.0,1.7 \mathrm{~Hz}, 1 \mathrm{H})$ $8.13(\mathrm{~d}, J=8.4 \mathrm{~Hz}, 1 \mathrm{H}), 8.07(\mathrm{~d}, J=8.1 \mathrm{~Hz}, 1 \mathrm{H}), 7.81-7.75(\mathrm{~m}, 2 \mathrm{H}), 7.59(\mathrm{dd}, J=8.0,4.7 \mathrm{~Hz}, 1 \mathrm{H})$, $7.52(\mathrm{t}, J=7.3 \mathrm{~Hz}, 1 \mathrm{H}), 6.82(\mathrm{~d}, J=3.8 \mathrm{~Hz}, 1 \mathrm{H}), 6.18(\mathrm{~s}, 1 \mathrm{H}), 5.74-5.64(\mathrm{~m}, 1 \mathrm{H}), 5.17(\mathrm{~d}, J=17.3$ $\mathrm{Hz}, 1 \mathrm{H}), 5.06(\mathrm{~d}, J=16.1 \mathrm{~Hz}, 1 \mathrm{H}), 4.93(\mathrm{~d}, J=10.6 \mathrm{~Hz}, 1 \mathrm{H}), 4.85(\mathrm{~d}, J=16.1 \mathrm{~Hz}, 1 \mathrm{H}), 4.51-4.41$ $(\mathrm{m}, 1 \mathrm{H}), 4.32-4.22(\mathrm{~m}, 2 \mathrm{H}), 4.04(\mathrm{t}, J=11.5 \mathrm{~Hz}, 1 \mathrm{H}), 3.82(\mathrm{t}, J=10.2 \mathrm{~Hz}, 1 \mathrm{H}), 2.88$ (bs, $1 \mathrm{H}), 2.18$ $-2.08(\mathrm{~m}, 2 \mathrm{H}), 2.07-1.98(\mathrm{~m}, 2 \mathrm{H}), 1.16(\mathrm{t}, J=11.1 \mathrm{~Hz}, 1 \mathrm{H})$ $\delta 163.9,150.1,147.6,147.0,144.8,144.3,138.0,135.4,130.5,129.9,129.4,126.9,124.2,123.7$, 123.0, 120.1, 115.9, 65.2, 64.9, 60.5, 59.1, 55.5, 36.9, 25.3, 24.7, 20.8.

Calc. for $\mathrm{C}_{26} \mathrm{H}_{28} \mathrm{~N}_{4} \mathrm{O}_{2} \mathrm{Cl}[\mathrm{M}]^{+}$: 463.1901 , found: 463.1902 $\delta 8.96(\mathrm{~d}, J=4.5 \mathrm{~Hz}, 1 \mathrm{H}), 8.43(\mathrm{~s}, 1 \mathrm{H}), 8.18(\mathrm{~d}, J=8.4 \mathrm{~Hz}, 1 \mathrm{H}), 8.08(\mathrm{~d}, J=8.3 \mathrm{~Hz}, 1 \mathrm{H}), 7.84-7.78$ (m, 1H), 7.75 (d, J=4.5 Hz, 1H), 7.64 (ddd, $J=8.2,6.9,1.0 \mathrm{~Hz}, 1 \mathrm{H}), 6.74(\mathrm{~d}, J=3.9 \mathrm{~Hz}, 1 \mathrm{H}), 5.96$ (s, $1 \mathrm{H}), 5.71-5.61(\mathrm{~m}, 1 \mathrm{H}), 5.19(\mathrm{~d}, J=17.3 \mathrm{~Hz}, 1 \mathrm{H}), 4.93(\mathrm{~d}, J=10.6 \mathrm{~Hz}, 1 \mathrm{H}), 4.58(\mathrm{~d}, J=16.0$ $\mathrm{Hz}, 1 \mathrm{H}), 4.39(\mathrm{~d}, J=15.9 \mathrm{~Hz}, 3 \mathrm{H}), 4.29(\mathrm{t}, J=9.6 \mathrm{~Hz}, 1 \mathrm{H}), 3.84(\mathrm{t}, J=11.4 \mathrm{~Hz}, 1 \mathrm{H}), 3.64(\mathrm{t}, J=10.6$ $\mathrm{Hz}, 1 \mathrm{H}), 2.84(\mathrm{~s}, 1 \mathrm{H}), 2.10-2.02(\mathrm{~m}, 11 \mathrm{H}), 1.99-1.91(\mathrm{~m}, 2 \mathrm{H}), 1.66(\mathrm{~s}, 6 \mathrm{H}), 1.08(\mathrm{t}, J=11.1 \mathrm{~Hz}$, $1 \mathrm{H})$.

$\delta 163.3,150.1,147.5,144.8,138.1,129.9,129.5,126.9,124.2,123.0,119.9,115.8,65.3,63.7$, 60.3, 58.7, 55.5, 52.2, 40.9 (x3), 36.8, 35.8 (x3), 28.7 (x3), 25.2, 24.7, 20.9. Calc. for $\mathrm{C}_{31} \mathrm{H}_{40} \mathrm{~N}_{3} \mathrm{O}_{2}[\mathrm{M}]^{+}:$: 486.3121, found: 486.3115 . 


\section{Compound 43}

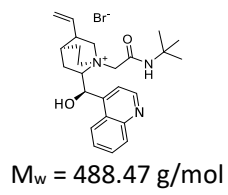

${ }^{1} \mathrm{H}$ NMR $\left(400 \mathrm{MHz}, \mathrm{DMSO}-d_{6}\right)$

${ }^{13} \mathrm{C}$ NMR $\left(100 \mathrm{MHz}, \mathrm{DMSO}-d_{6}\right)$

HRMS ESI $(\mathrm{m} / \mathrm{z})$
Following Procedure B and using 21 (0.7 g, $3.4 \mathrm{mmol})$, the product 43 (1.6 g, $3.3 \mathrm{mmol}, \mathbf{9 8 \%})$ was obtained as colorless solid $\left(\mathrm{mp} 214-215^{\circ} \mathrm{C} ;[\alpha]_{\mathrm{D}}^{20}=-74.8(c=1.1, \mathrm{MeOH})\right)$

\section{Compound $\mathbf{4 4}$}

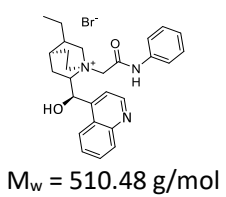

${ }^{1} \mathrm{H}$ NMR $\left(400 \mathrm{MHz}, \mathrm{DMSO}-d_{6}\right)$

${ }^{13} \mathrm{C}$ NMR (100 MHz, DMSO- $\left.d_{6}\right)$

HRMS ESI $(\mathrm{m} / \mathrm{z})$
Following Procedure B and using 1 (0.7 g, $3.4 \mathrm{mmol})$, the product $44(1.4 \mathrm{~g}, 3.3 \mathrm{mmol}, \mathbf{9 3 \%})$ was obtained as colorless solid $\left(\mathrm{mp} 153-154^{\circ} \mathrm{C} ;[\alpha]_{\mathrm{D}}^{20}=-39.8(c=0.9, \mathrm{MeOH})\right)$.

\section{Compound 45}

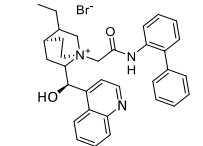

$\mathrm{M}_{\mathrm{w}}=586.57 \mathrm{~g} / \mathrm{mol}$

${ }^{1} \mathrm{H}$ NMR $\left(400 \mathrm{MHz}, \mathrm{DMSO}-d_{6}\right)$

${ }^{13} \mathrm{C}$ NMR $\left(100 \mathrm{MHz}\right.$, DMSO- $\left.d_{6}\right)$

HRMS ESI (m/z) $\delta 8.96(\mathrm{~d}, J=4.4 \mathrm{~Hz}, 1 \mathrm{H}), 8.56(\mathrm{~s}, 1 \mathrm{H}), 8.21(\mathrm{~d}, J=8.4 \mathrm{~Hz}, 1 \mathrm{H}), 8.08(\mathrm{~d}, J=8.3 \mathrm{~Hz}, 1 \mathrm{H}), 7.82-7.73$ $(\mathrm{m}, 2 \mathrm{H}), 7.65(\mathrm{t}, J=7.5 \mathrm{~Hz}, 1 \mathrm{H}), 6.73(\mathrm{~d}, J=3.7 \mathrm{~Hz}, 1 \mathrm{H}), 5.98(\mathrm{~s}, 1 \mathrm{H}), 5.73-5.59(\mathrm{~m}, 1 \mathrm{H}), 5.19(\mathrm{~d}$, $J=17.3 \mathrm{~Hz}, 1 \mathrm{H}), 4.93(\mathrm{~d}, J=10.5 \mathrm{~Hz}, 1 \mathrm{H}), 4.61(\mathrm{~d}, J=15.9 \mathrm{~Hz}, 1 \mathrm{H}), 4.44-4.27(\mathrm{~m}, 4 \mathrm{H}), 3.87(\mathrm{t}, J$ $=11.3 \mathrm{~Hz}, 1 \mathrm{H}), 3.67(\mathrm{t}, J=10.1 \mathrm{~Hz}, 1 \mathrm{H}), 2.84(\mathrm{bs}, 1 \mathrm{H}), 2.07(\mathrm{~s}, 2 \mathrm{H}), 2.00-1.91(\mathrm{~m}, 2 \mathrm{H}), 1.39(\mathrm{~s}$, 9H), $1.07(\mathrm{t}, J=10.7 \mathrm{~Hz}, 1 \mathrm{H})$.

$\delta 163.5,150.1,147.5,144.8,138.1,129.9,129.4,127.0,124.2,123.0,119.9,115.7,65.3,63.9$, $60.2,58.8,55.5,51.5,36.8,28.3,25.2,24.6,20.9$.

Calc. for $\mathrm{C}_{25} \mathrm{H}_{34} \mathrm{~N}_{3} \mathrm{O}_{2}[\mathrm{M}]^{+}$: 408.2651, found: 408.2649 . $\delta 11.07(\mathrm{~s}, 1 \mathrm{H}), 8.97(\mathrm{~d}, J=4.4 \mathrm{~Hz}, 1 \mathrm{H}), 8.17(\mathrm{~d}, J=8.4 \mathrm{~Hz}, 1 \mathrm{H}), 8.07(\mathrm{~d}, J=8.3 \mathrm{~Hz}, 1 \mathrm{H}), 7.82-$ $7.75(\mathrm{~m}, 4 \mathrm{H}), 7.51(\mathrm{t}, J=7.6 \mathrm{~Hz}, 1 \mathrm{H}), 7.44(\mathrm{t}, J=7.8 \mathrm{~Hz}, 2 \mathrm{H}), 7.20(\mathrm{t}, J=7.4 \mathrm{~Hz}, 1 \mathrm{H}), 6.71(\mathrm{~d}, J=$ $3.7 \mathrm{~Hz}, 1 \mathrm{H}), 6.18$ (bs, $1 \mathrm{H}), 4.88(\mathrm{~d}, J=15.9 \mathrm{~Hz}, 1 \mathrm{H}), 4.72$ (d, $J=15.8 \mathrm{~Hz}, 1 \mathrm{H}), 4.40$ (t, $J=11.4 \mathrm{~Hz}$ $1 \mathrm{H}), 4.26(\mathrm{t}, J=9.0 \mathrm{~Hz}, 1 \mathrm{H}), 3.98(\mathrm{t}, J=11.3 \mathrm{~Hz}, 1 \mathrm{H}), 3.89(\mathrm{~d}, J=11.5 \mathrm{~Hz}, 1 \mathrm{H}), 3.75$ (dd, $J=11.3$, $7.0 \mathrm{~Hz}, 1 \mathrm{H}), 2.16-1.86(\mathrm{~m}, 5 \mathrm{H}), 1.34-1.13(\mathrm{~m}, 3 \mathrm{H}), 0.72(\mathrm{t}, J=7.3 \mathrm{~Hz}, 3 \mathrm{H})$. $\delta 162,5,150,1,147,6,144,9,137,7,129,9,129,3,129,0,127,0,124,6,124,3,123,0,120,2,119,7$, $65,1,64,9,63,8,59,5,55,5,35,5,25,7,25,4,23,4,20,2,11,3$. Calc. for $\mathrm{C}_{27} \mathrm{H}_{32} \mathrm{~N}_{3} \mathrm{O}_{2}[\mathrm{M}]^{+}:$:30.2495, found: 430.2476 .

Following Procedure $\mathbf{B}$ and using $8(1.0 \mathrm{~g}, 3.4 \mathrm{mmol})$, the product $45(1.9 \mathrm{~g}, 3.2 \mathrm{mmol}, \mathbf{9 2} \%)$ was obtained as colorless solid $\left(\mathrm{mp} 152-153^{\circ} \mathrm{C} ;[\alpha]_{\mathrm{D}}^{20}=-15.1(c=1.0, \mathrm{MeOH})\right)$.

$\delta 10.44(\mathrm{~s}, 1 \mathrm{H}), 8.97(\mathrm{~d}, J=4.4 \mathrm{~Hz}, 1 \mathrm{H}), 8.10(\mathrm{dd}, J=19.6,8.4 \mathrm{~Hz}, 2 \mathrm{H}), 7.82-7.73(\mathrm{~m}, 2 \mathrm{H}), 7.62$ $-7.53(\mathrm{~m}, 2 \mathrm{H}), 7.52-7.35(\mathrm{~m}, 8 \mathrm{H}), 7.27(\mathrm{t}, J=7.3 \mathrm{~Hz}, 1 \mathrm{H}), 6.67(\mathrm{~d}, J=3.7 \mathrm{~Hz}, 1 \mathrm{H}), 6.00(\mathrm{~s}, 1 \mathrm{H})$ $4.67(\mathrm{~d}, J=15.6 \mathrm{~Hz}, 1 \mathrm{H}), 4.46(\mathrm{~d}, J=15.6 \mathrm{~Hz}, 1 \mathrm{H}), 4.34-4.25(\mathrm{~m}, 1 \mathrm{H}), 4.19(\mathrm{t}, J=8.7 \mathrm{~Hz}, 1 \mathrm{H})$, $3.70-3.54(\mathrm{~m}, 2 \mathrm{H}), 3.47-3.40(\mathrm{~m}, 1 \mathrm{H}), 2.07(\mathrm{t}, J=11.1 \mathrm{~Hz}, 1 \mathrm{H}), 2.01-1.91(\mathrm{~m}, 2 \mathrm{H}), 1.90-1.78$ $(\mathrm{m}, 2 \mathrm{H}), 1.20-1.04(\mathrm{~m}, 3 \mathrm{H}), 0.69(\mathrm{t}, J=7.3 \mathrm{~Hz}, 3 \mathrm{H})$.

ठ $162.9,150.1,147.6,145.0,138.7,137.8,133.0,130.6,129.8,129.4,128.6,128.5,128.1,127.4$ 127.2 (x2), 127.0, 124.3, 123.3, 120.0, 65.3, 65.1, 63.2, 59.2, 55.1, 35.5, 25.6, 25.3, 23.2, 20.3, 11.3 .

Calc. for $\mathrm{C}_{33} \mathrm{H}_{36} \mathrm{~N}_{3} \mathrm{O}_{2}[\mathrm{M}]^{+}:$506.2808, found: 506.2814 . 


\section{Compound 46}

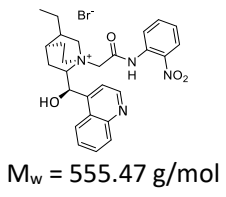

${ }^{1} \mathrm{H}$ NMR $\left(400 \mathrm{MHz}, \mathrm{DMSO}-d_{6}\right)$

${ }^{13} \mathrm{C}$ NMR (100 MHz, DMSO- $\left.d_{6}\right)$

HRMS ESI $(\mathrm{m} / \mathrm{z})$
Following Procedure B and using 10 (0.9 g, $3.4 \mathrm{mmol})$, the product 46 (1.8 g, $3.3 \mathrm{mmol}, \mathbf{9 2 \%})$ was obtained as yellowish solid $\left(\mathrm{mp} 146-148^{\circ} \mathrm{C} ;[\alpha]_{\mathrm{D}}^{20}=-14.4(c=1.0, \mathrm{MeOH})\right)$.

$\delta 11.38(\mathrm{~s}, 1 \mathrm{H}), 8.98(\mathrm{~d}, J=4.4 \mathrm{~Hz}, 1 \mathrm{H}), 8.16(\mathrm{~d}, J=8.4 \mathrm{~Hz}, 1 \mathrm{H}), 8.08(\mathrm{~d}, J=8.3 \mathrm{~Hz}, 2 \mathrm{H}), 7.85(\mathrm{t}, J$ $=7.3 \mathrm{~Hz}, 1 \mathrm{H}), 7.82-7.76(\mathrm{~m}, 2 \mathrm{H}), 7.70(\mathrm{~d}, J=7.8 \mathrm{~Hz}, 1 \mathrm{H}), 7.56(\mathrm{t}, J=5.0 \mathrm{~Hz}, 2 \mathrm{H}), 6.79(\mathrm{~d}, J=3.7$ $\mathrm{Hz}, 1 \mathrm{H}), 6.18(\mathrm{bs}, 1 \mathrm{H}), 4.88(\mathrm{~d}, J=15.9 \mathrm{~Hz}, 1 \mathrm{H}), 4.70(\mathrm{~d}, J=15.9 \mathrm{~Hz}, 1 \mathrm{H}), 4.42(\mathrm{t}, J=12.5 \mathrm{~Hz}, 1 \mathrm{H})$ $4.24(\mathrm{t}, J=9.0 \mathrm{~Hz}, 1 \mathrm{H}), 3.95(\mathrm{t}, J=11.2 \mathrm{~Hz}, 1 \mathrm{H}), 3.75-3.62(\mathrm{~m}, 2 \mathrm{H}), 2.13-1.88(\mathrm{~m}, 5 \mathrm{H}), 1.27-$ $1.08(\mathrm{~m}, 3 \mathrm{H}), 0.72(\mathrm{t}, J=7.3 \mathrm{~Hz}, 3 \mathrm{H})$.

$\delta 163.2,150.1,149.5,147.6,144.9,143.4,134.3,129.8,129.4,129.1,127.0,126.3,125.2,124.4$, $123.2,120.1,65.4,65.1,63.5,59.3,55.6,35.5,25.7,23.4,20.4,19.8,11.2$.

Calc. for $\mathrm{C}_{27} \mathrm{H}_{31} \mathrm{~N}_{4} \mathrm{O}_{4}[\mathrm{M}]^{+}$: 475.2345, found: 475.2341 .

\section{Compound 47}

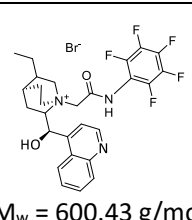

${ }^{1} \mathrm{H}$ NMR (400 MHz, DMSO- $\left.d_{6}\right)$

${ }^{13} \mathrm{C}$ NMR $\left(100 \mathrm{MHz}, \mathrm{DMSO}-d_{6}\right)$

HRMS ESI $(m / z)$
Following Procedure B and using 16 (1.0 g, $3.4 \mathrm{mmol})$, the product 47 (1.9 g, $3.2 \mathrm{mmol}, \mathbf{9 4 \%})$ was obtained as colorless solid $\left(\mathrm{mp} 188-190^{\circ} \mathrm{C} ;[\alpha]_{\mathrm{D}}^{20}=-17.7(c=1.0, \mathrm{MeOH})\right)$.

\section{Compound 49}

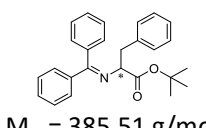

${ }^{1} \mathbf{H}$ NMR $\left(400 \mathrm{MHz}, \mathrm{CDCl}_{3}\right)$

${ }^{13} \mathrm{C}$ NMR $\left(100 \mathrm{MHz}, \mathrm{CDCl}_{3}\right)$

HPLC
Following Procedure $\mathbf{C}$, using catalyst $46(6.7 \mathrm{mg}, 0.012 \mathrm{mmol})$, and benzyl bromide $(120 \mu \mathrm{L}, 1.0$ $\mathrm{mmol})$, the product $49(72 \mathrm{mg}, 0.19 \mathrm{mmol}, 94 \%)$ was obtained as colorless oil; $[\alpha]_{\mathrm{D}}^{20}=$ $-220.9\left(c=0.5, \mathrm{CHCl}_{3}\right)$. Analytical data are in full accordance with those reported in literature. $^{3}$

$\delta 11.30(\mathrm{~s}, 1 \mathrm{H}), 8.98(\mathrm{~d}, J=4.4 \mathrm{~Hz}, 1 \mathrm{H}), 8.10(\mathrm{t}, J=8.9 \mathrm{~Hz}, 2 \mathrm{H}), 7.84-7.76(\mathrm{~m}, 2 \mathrm{H}), 7.58(\mathrm{t}, J=7.4$ $\mathrm{Hz}, 1 \mathrm{H}), 6.80(\mathrm{~d}, J=3.4 \mathrm{~Hz}, 1 \mathrm{H}), 6.13(\mathrm{bs}, 1 \mathrm{H}), 4.98(\mathrm{~d}, J=16.2 \mathrm{~Hz}, 1 \mathrm{H}), 4.89(\mathrm{~d}, J=16.4 \mathrm{~Hz}, 1 \mathrm{H})$, $4.43(\mathrm{t}, J=10.9 \mathrm{~Hz}, 1 \mathrm{H}), 4.27(\mathrm{t}, J=8.2 \mathrm{~Hz}, 1 \mathrm{H}), 3.98(\mathrm{t}, J=11.2 \mathrm{~Hz}, 1 \mathrm{H}), 3.79-3.70(\mathrm{~m}, 2 \mathrm{H}), 2.15$ $-1.85(\mathrm{~m}, 5 \mathrm{H}), 1.30-1.11(\mathrm{~m}, 3 \mathrm{H}), 0.71(\mathrm{t}, J=7.2 \mathrm{~Hz}, 3 \mathrm{H})$.

$\delta 163.7,150.2,147.6,144.7,129.9,129.5,127.0,124.3,123.0,120.2,65.4,65.0,63.4,58.6$, $55.5,35.5,25.7,25.3,23.4,20.4,11.2$.

Calc. for $\mathrm{C}_{27} \mathrm{H}_{27} \mathrm{~N}_{3} \mathrm{O}_{2} \mathrm{~F}_{5}[\mathrm{M}]^{+}: 520.2023$, found: 520.2001 .
ס $7.53-7.47(\mathrm{~m}, 2 \mathrm{H}), 7.31-7.17(\mathrm{~m}, 6 \mathrm{H}), 7.13-7.06(\mathrm{~m}, 3 \mathrm{H}), 7.00-6.95(\mathrm{~m}, 2 \mathrm{H}), 6.54(\mathrm{~d}, J=$ $6.9 \mathrm{~Hz}, 2 \mathrm{H}), 4.03(\mathrm{dd}, J=9.2,4.4 \mathrm{~Hz}, 1 \mathrm{H}), 3.20-3.04(\mathrm{~m}, 2 \mathrm{H}), 1.36(\mathrm{~s}, 9 \mathrm{H})$.

$\delta 171.0,170.4,139.8,138.5,137.6,136.6,132.6,130.2,130.0,129.5,128.9,128.7,128.5$ $128.3,128.2$ (x2) 128.1, 127.8, $126.9126 .3,81.2,68.1,39.8,28.2$.

Daicel Chiralpak OD-H, 99.5:0.5 $n$-hexane/i-PrOH, $254 \mathrm{~nm}, 1.0 \mathrm{~mL} / \mathrm{min}, t_{R}=13.9 \mathrm{~min}(R), t_{R}=$ $20.0 \mathrm{~min}(\mathrm{~S})$ (92\% ee). Absolute configuration was determined by comparison of the retention times and the $[\alpha] D$-value with reported data $^{3}$ 


\section{Compound $\mathbf{5 0}$}

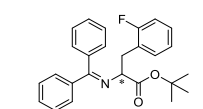

$\mathrm{M}_{\mathrm{w}}=403.50 \mathrm{~g} / \mathrm{mol}$

${ }^{1} \mathbf{H}$ NMR $\left(400 \mathrm{MHz}, \mathrm{CDCl}_{3}\right)$

${ }^{13} \mathrm{C}$ NMR (100 MHz, $\mathrm{CDCl}_{3}$ )

HPLC

\section{Compound $\mathbf{5 1}$}

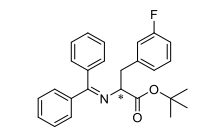

$\mathrm{M}_{\mathrm{w}}=403.50 \mathrm{~g} / \mathrm{mol}$

${ }^{1} \mathbf{H}$ NMR (400 MHz, $\left.\mathrm{CDCl}_{3}\right)$

${ }^{13} \mathrm{C}$ NMR (100 MHz, $\left.\mathrm{CDCl}_{3}\right)$

HPLC

\section{Compound 52}

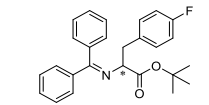

$\mathrm{M}_{\mathrm{w}}=403.50 \mathrm{~g} / \mathrm{mol}$

${ }^{1} \mathbf{H}$ NMR (400 MHz, $\mathrm{CDCl}_{3}$ )

${ }^{13} \mathrm{C}$ NMR (100 MHz, $\left.\mathrm{CDCl}_{3}\right)$

HPLC
Following Procedure C, using catalyst $46(6.7 \mathrm{mg}, 0.012 \mathrm{mmol}$ ), and 2-fluorobenzyl bromide (120 $\mu \mathrm{L}, 1.0 \mathrm{mmol})$, the product $\mathbf{5 0}(78 \mathrm{mg}, 0.19 \mathrm{mmol}, \mathbf{9 7 \%})$ was obtained as colorless oil; $[\alpha]_{\mathrm{D}}^{20}=$ $-180.4\left(c=0.7, \mathrm{CHCl}_{3}\right)$. Analytical data are in full accordance with those reported in literature. ${ }^{3}$

$\delta 7.51-7.48(\mathrm{~m}, 2 \mathrm{H}), 7.31-7.25(\mathrm{~m}, 2 \mathrm{H}), 7.24-7.20(\mathrm{~m}, 4 \mathrm{H}), 7.09-7.04(\mathrm{~m}, 2 \mathrm{H}), 6.91-6.80$ $(\mathrm{m}, 2 \mathrm{H}), 6.61(\mathrm{~d}, J=7.0 \mathrm{~Hz}, 2 \mathrm{H}), 4.12(\mathrm{dd}, J=9.2,4.5 \mathrm{~Hz}, 1 \mathrm{H}), 3.26(\mathrm{dd}, J=13.5,4.3 \mathrm{~Hz}, 1 \mathrm{H}), 3.07$ (dd, $J=13.4,9.4 \mathrm{~Hz}, 1 \mathrm{H}), 1.36(\mathrm{~s}, 9 \mathrm{H})$.

$\delta 170.7,170.6,163.1,159.9,139.7,136.4,132.6,130.3,128.9,128.5$ (x2), $128.2(x 2), 128.1$ (x2), 127.8 (x2), 125.6, 125.4, 123.8, 123.7, 115.3, 115.0, 81.4, 66.3, 32.9, 28.2.

Daicel Chiralpak OD-H, 99.5:0.5 $n$-hexane/i-PrOH, $254 \mathrm{~nm}, 1.0 \mathrm{~mL} / \mathrm{min}, t_{R}=16.6 \min (R), t_{R}=$ $17.5 \mathrm{~min}(S)(94 \%$ ee). Absolute configuration was determined by comparison of the retention times with reported data ${ }^{3}$

Following Procedure C, using catalyst 46 (6.7 $\mathrm{mg}, 0.012 \mathrm{mmol}$ ), and 3-fluorobenzyl bromide (123 $\mu \mathrm{L}, 1.0 \mathrm{mmol})$, the product $51(74 \mathrm{mg}, 0.18 \mathrm{mmol}, \mathbf{9 2 \%})$ was obtained as colorless oil; $[\alpha]_{\mathrm{D}}^{20}=$ $-141.3\left(c=0.6, \mathrm{CHCl}_{3}\right)$. Analytical data are in full accordance with those reported in literature. ${ }^{3}$

$\delta 7.50(\mathrm{dd}, J=5.3,3.3 \mathrm{~Hz}, 2 \mathrm{H}), 7.31-7.20(\mathrm{~m}, 6 \mathrm{H}), 7.11-7.03(\mathrm{~m}, 1 \mathrm{H}), 6.81-6.74(\mathrm{~m}, 2 \mathrm{H}), 6.71$ $-6.66(\mathrm{~m}, 1 \mathrm{H}), 6.62(\mathrm{~d}, J=6.9 \mathrm{~Hz}, 2 \mathrm{H}), 4.04(\mathrm{dd}, J=9.0,4.4 \mathrm{~Hz}, 1 \mathrm{H}), 3.18-3.03(\mathrm{~m}, 2 \mathrm{H}), 1.37(\mathrm{~s}$, $9 \mathrm{H})$.

$\delta 170.7$ (x2), 164.5, 161.1, 141.2, 141.1, 139.6, 136.4, 132.5, 130.4, 129.6, 129.5, 128.9, 128.5 $128.3,128.1,127.8,125.7$ (x2), 116.8, 116.6, 113.3, 113.1, 81.5, 67.7, 39.5, 28.2.

Daicel Chiralpak OD-H, 99.5:0.5 $n$-hexane/i-PrOH, $254 \mathrm{~nm}, 1.0 \mathrm{~mL} / \mathrm{min}, t_{R}=16.3 \mathrm{~min}(\mathrm{~S}), t_{R}=21.8$ $\min (R)(86 \%$ ee). Absolute configuration was determined by comparison of the retention times and the $[\alpha] \mathrm{D}$-value with reported data $^{3}$

Following Procedure C, using catalyst $46(6.7 \mathrm{mg}, 0.012 \mathrm{mmol}$ ), and 4-fluorobenzyl bromide (125 $\mu \mathrm{L}, 1.0 \mathrm{mmol})$, the product $52(76 \mathrm{mg}, 0.19 \mathrm{mmol}, \mathbf{9 4 \%})$ was obtained as colorless oil; $[\alpha]_{\mathrm{D}}^{20}=$ $-174.6\left(c=0.6, \mathrm{CHCl}_{3}\right)$. Analytical data are in full accordance with those reported in literature. ${ }^{3}$

$\delta 7.52-7.47(\mathrm{~m}, 2 \mathrm{H}), 7.31-7.20(\mathrm{~m}, 6 \mathrm{H}), 6.97-6.91(\mathrm{~m}, 2 \mathrm{H}), 6.83-6.76(\mathrm{~m}, 2 \mathrm{H}), 6.60(\mathrm{~d}, J=$ $6.9 \mathrm{~Hz}, 2 \mathrm{H}), 4.01(\mathrm{dd}, J=9.0,4.5 \mathrm{~Hz}, 1 \mathrm{H}), 3.15-3.02(\mathrm{~m}, 2 \mathrm{H}), 1.36(\mathrm{~s}, 9 \mathrm{H})$.

$\delta 170.8,170.6,162.9,160.5,139.6,136.5,134.3,134.2,131.4(x 2), 131.1$ (x2), 130.5, 130.3, $128.9,128.5,128.4,128.3,128.1,127.8,115.0,114.8,81.4,68.0,38.9,28.2$.

Daicel Chiralpak OD-H, 99.5:0.5 $n$-hexane/i-PrOH, $254 \mathrm{~nm}, 1.0 \mathrm{~mL} / \mathrm{min}, t_{R}=14.1 \mathrm{~min}(\mathrm{~S}), t_{R}=21.1$ $\min (R)(91 \%$ ee). Absolute configuration was determined by comparison of the retention times and the $[\alpha] D-v a l u e$ with reported data ${ }^{3}$ 


\section{Compound 53}

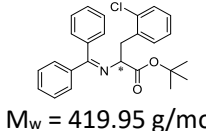

${ }^{1} \mathbf{H}$ NMR $\left(400 \mathrm{MHz}, \mathrm{CDCl}_{3}\right)$

${ }^{13} \mathrm{C}$ NMR $\left(100 \mathrm{MHz}, \mathrm{CDCl}_{3}\right)$

HPLC
Following Procedure C, using catalyst $46(6.7 \mathrm{mg}, 0.012 \mathrm{mmol})$, and 2-chlorobenzyl bromide $(130 \mu \mathrm{L}, 1.0 \mathrm{mmol})$, the product $53(77 \mathrm{mg}, 0.18 \mathrm{mmol}, 92 \%)$ was obtained as colorless oil; $[\alpha]_{\mathrm{D}}^{20}=-181.5\left(c=0.5, \mathrm{CHCl}_{3}\right)$. Analytical data are in full accordance with those reported in literature. $^{4}$

\section{Compound 54}

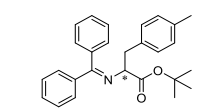

$\mathrm{M}_{\mathrm{w}}=399.53 \mathrm{~g} / \mathrm{mol}$

${ }^{13} \mathrm{C}$ NMR $\left(100 \mathrm{MHz}, \mathrm{CDCl}_{3}\right)$

HPLC

\section{Compound 55}

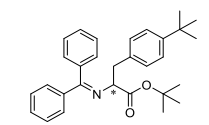

$\mathrm{M}_{\mathrm{w}}=441.62 \mathrm{~g} / \mathrm{mol}$

${ }^{1} \mathbf{H}$ NMR $\left(400 \mathrm{MHz}, \mathrm{CDCl}_{3}\right)$

${ }^{13} \mathrm{C}$ NMR $\left(100 \mathrm{MHz}, \mathrm{CDCl}_{3}\right)$

HPLC

Following Procedure C, using catalyst $46(6.7 \mathrm{mg}, 0.012 \mathrm{mmol})$, and 4-methylbenzyl bromide (185 mg, $1.0 \mathrm{mmol})$, the product $54(76 \mathrm{mg}, 0.19 \mathrm{mmol}, 95 \%)$ was obtained as colorless oil; $[\alpha]_{\mathrm{D}}^{20}=-150.4\left(c=0.7, \mathrm{CHCl}_{3}\right)$. Analytical data are in full accordance with those reported in literature. ${ }^{3}$

$\delta 7.53-7.48(\mathrm{~m}, 2 \mathrm{H}), 7.31-7.19(\mathrm{~m}, 6 \mathrm{H}), 6.89(\mathrm{dd}, J=21.2,8.0 \mathrm{~Hz}, 4 \mathrm{H}), 6.57(\mathrm{~d}, J=7.0 \mathrm{~Hz}, 2 \mathrm{H})$ 4.02 (dd, $J=9.1,4.4 \mathrm{~Hz}, 1 \mathrm{H}$ ), 3.12 (dd, $J=13.4,4.4 \mathrm{~Hz}, 1 \mathrm{H}$ ), 3.03 (dd, $J=13.4,9.1 \mathrm{~Hz}, 1 \mathrm{H}$ ), 2.21 $(\mathrm{s}, 3 \mathrm{H}), 1.36(\mathrm{~s}, 9 \mathrm{H})$.

$\delta 171.1,170.3,139.8,137.8,136.6,135.7,135.4,132.6,130.2,129.9,128.9,128.3,128.2$

$128.1,127.8(x 2), 81.2,68.2,39.4,28.2,21.2$

Daicel Chiralpak OD-H, 99.5:0.5 $n$-hexane/i-PrOH, $254 \mathrm{~nm}, 1.0 \mathrm{~mL} / \mathrm{min}, t_{R}=14.5 \mathrm{~min}(\mathrm{~S}), t_{R}=17.6$ $\min (R)(90 \%$ ee). Absolute configuration was determined by comparison of the retention times and the $[\alpha] D$-value with reported data $^{3}$

Following Procedure C, using catalyst $46(6.7 \mathrm{mg}, 0.012 \mathrm{mmol})$, and 4-tert-butylbenzyl bromide $(184 \mu \mathrm{L}, 1.0 \mathrm{mmol})$, the product $55(86 \mathrm{mg}, 0.19 \mathrm{mmol}, \mathbf{9 7 \%})$ was obtained as colorless oil; $[\alpha]_{\mathrm{D}}^{20}=-118.5\left(c=0.6, \mathrm{CHCl}_{3}\right)$. Analytical data are in full accordance with those reported in literature. ${ }^{6}$

$\delta 7.53-7.49(\mathrm{~m}, 2 \mathrm{H}), 7.33-7.21(\mathrm{~m}, 4 \mathrm{H}), 7.18-7.12(\mathrm{~m}, 4 \mathrm{H}), 6.90(\mathrm{~d}, J=8.2 \mathrm{~Hz}, 2 \mathrm{H}), 6.47(\mathrm{~d}, J$ $=6.9 \mathrm{~Hz}, 2 \mathrm{H}), 4.00(\mathrm{dd}, J=9.2,4.2 \mathrm{~Hz}, 1 \mathrm{H}), 3.12(\mathrm{dd}, J=13.4,4.2 \mathrm{~Hz}, 1 \mathrm{H}), 3.03(\mathrm{dd}, J=13.4,9.2$ $\mathrm{Hz}, 1 \mathrm{H}), 1.36(\mathrm{~s}, 9 \mathrm{H}), 1.22(\mathrm{~s}, 9 \mathrm{H})$.

$\delta 171.2,170.3,149.3,139.8,137.8,136.6,135.5,130.2,129.7,128.9,128.2,128.1$ (x2), 127.8 , $125.1,81.2,68.3,39.2,34.5,31.6,28.2$.

Daicel Chiralpak OD-H, 99.5:0.5 $n$-hexane/i-PrOH, $254 \mathrm{~nm}, 1.0 \mathrm{~mL} / \mathrm{min}, t_{R}=12.3 \mathrm{~min}(\mathrm{~S}), t_{R}=14.9$ $\min (R)(83 \%$ ee). Absolute configuration was determined by comparison of the retention times and the $[\alpha] D$-value with reported data ${ }^{6}$ 


\section{Copies of ${ }^{1} \mathrm{H}$ and ${ }^{13} \mathrm{C}$ NMR spectra}

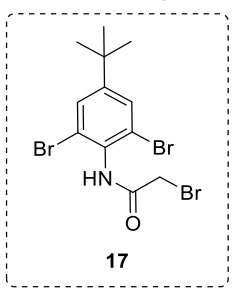

กิ

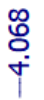

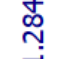

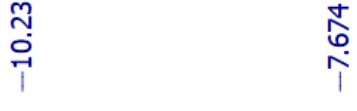

ب)

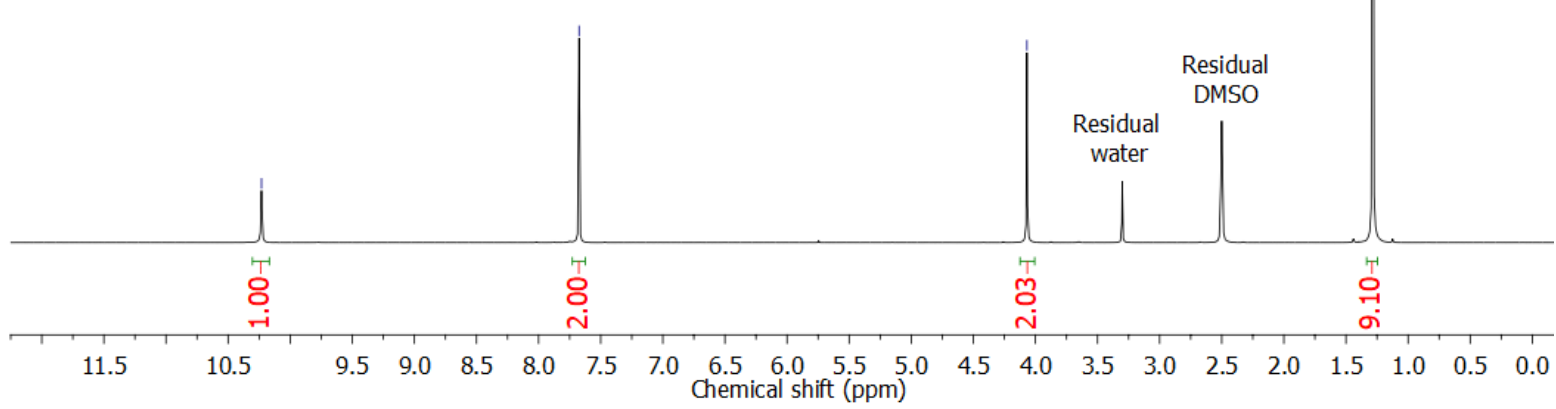

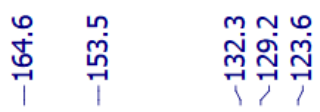

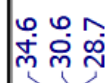

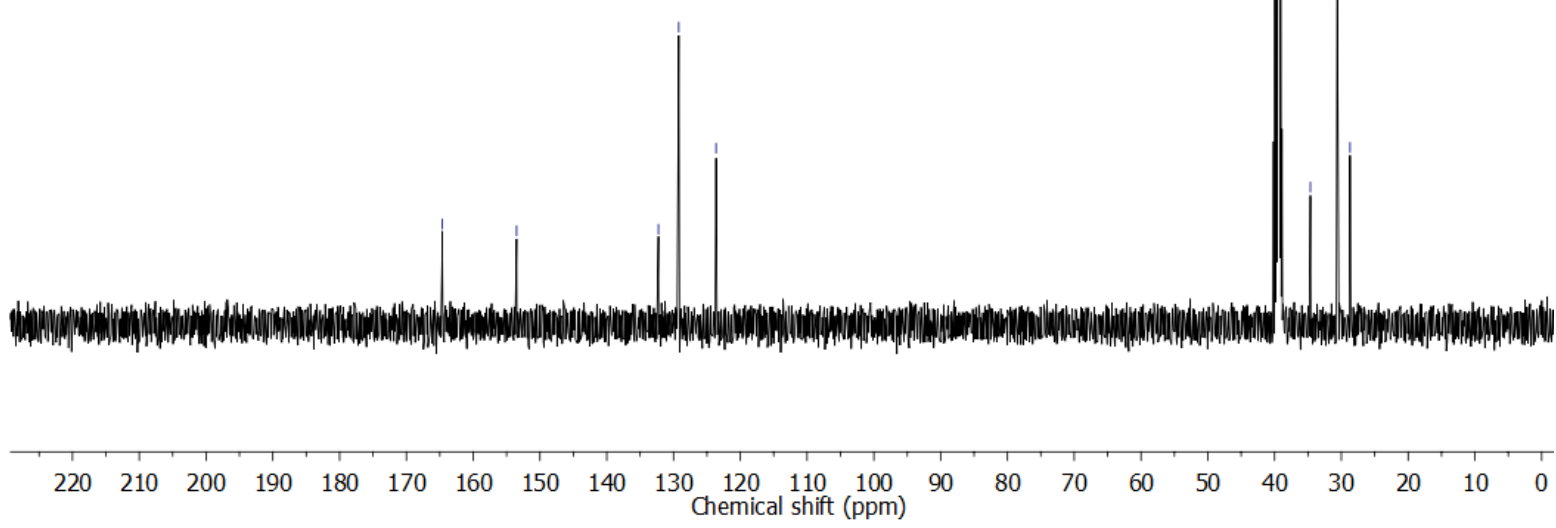

Fig. S3 ${ }^{1} \mathrm{H}$ NMR (400 MHz) and ${ }^{13} \mathrm{C}$ NMR (100 MHz) spectra of compound 17 in DMSO-d. 

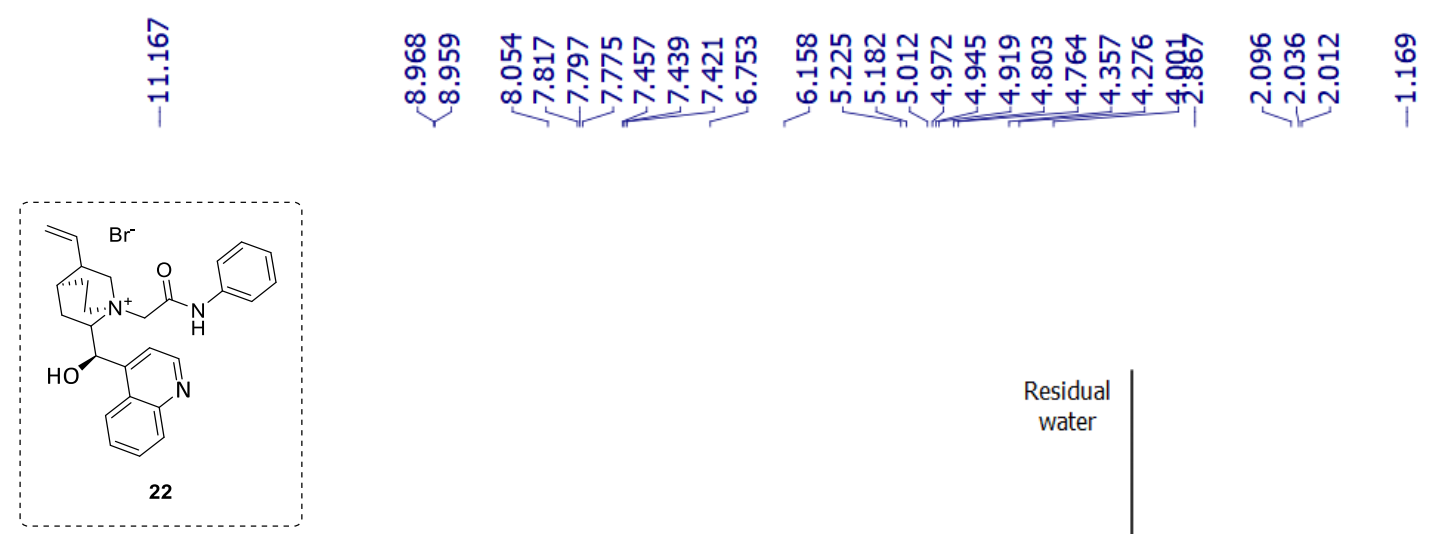

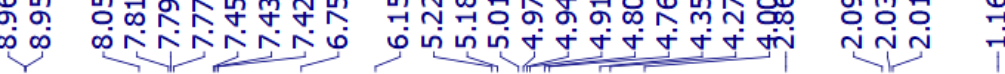

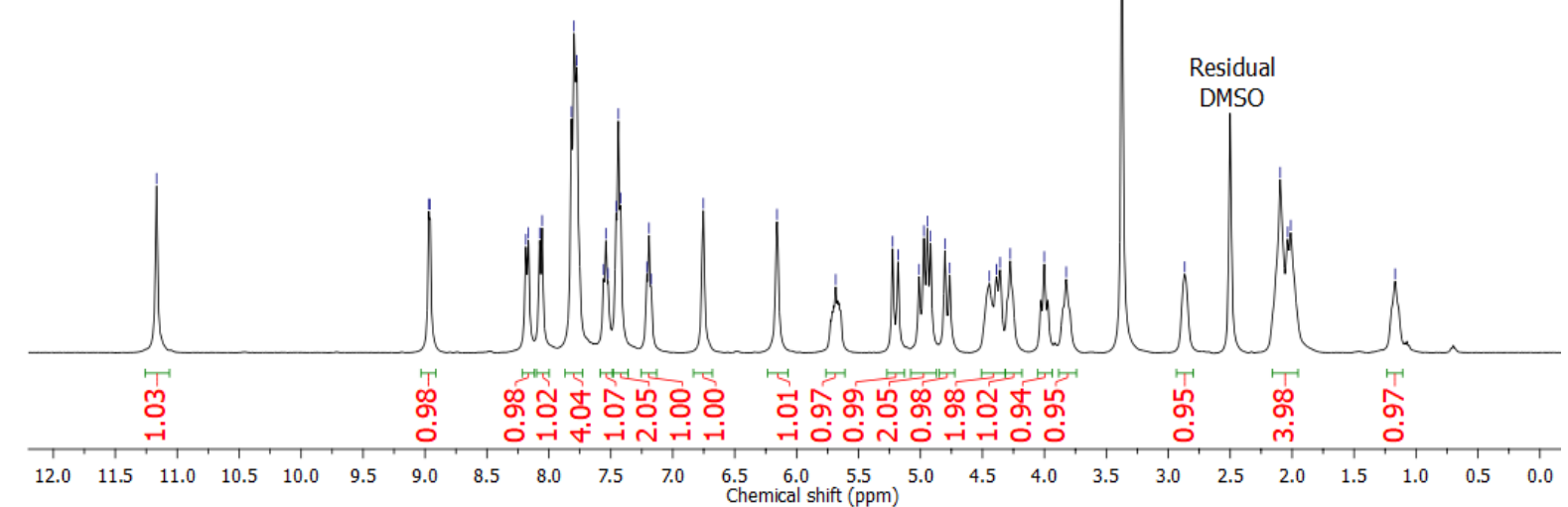

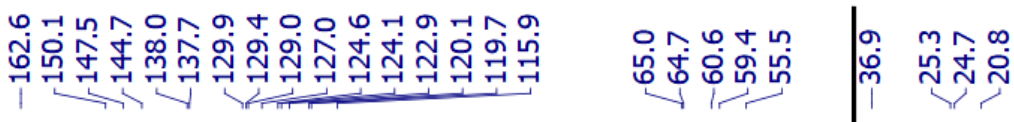

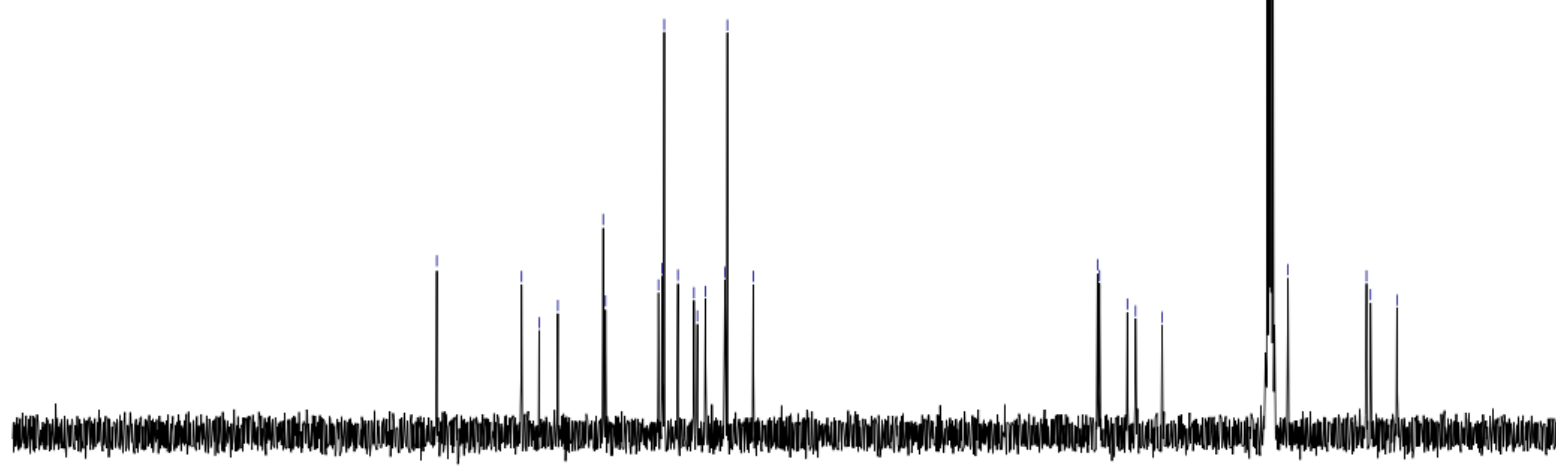

$\begin{array}{llllllllllllllllllllllll} & 220 & 210 & 200 & 190 & 180 & 170 & 160 & 150 & 140 & 130 & 120 & 110 & 100 & 90 & 80 & 70 & 60 & 50 & 40 & 30 & 20 & 10 & 0\end{array}$

Fig. S4 ${ }^{1} \mathrm{H}$ NMR (400 MHz) and ${ }^{13} \mathrm{C}$ NMR (100 MHz) spectra of compound 22 in DMSO-d 6 . 


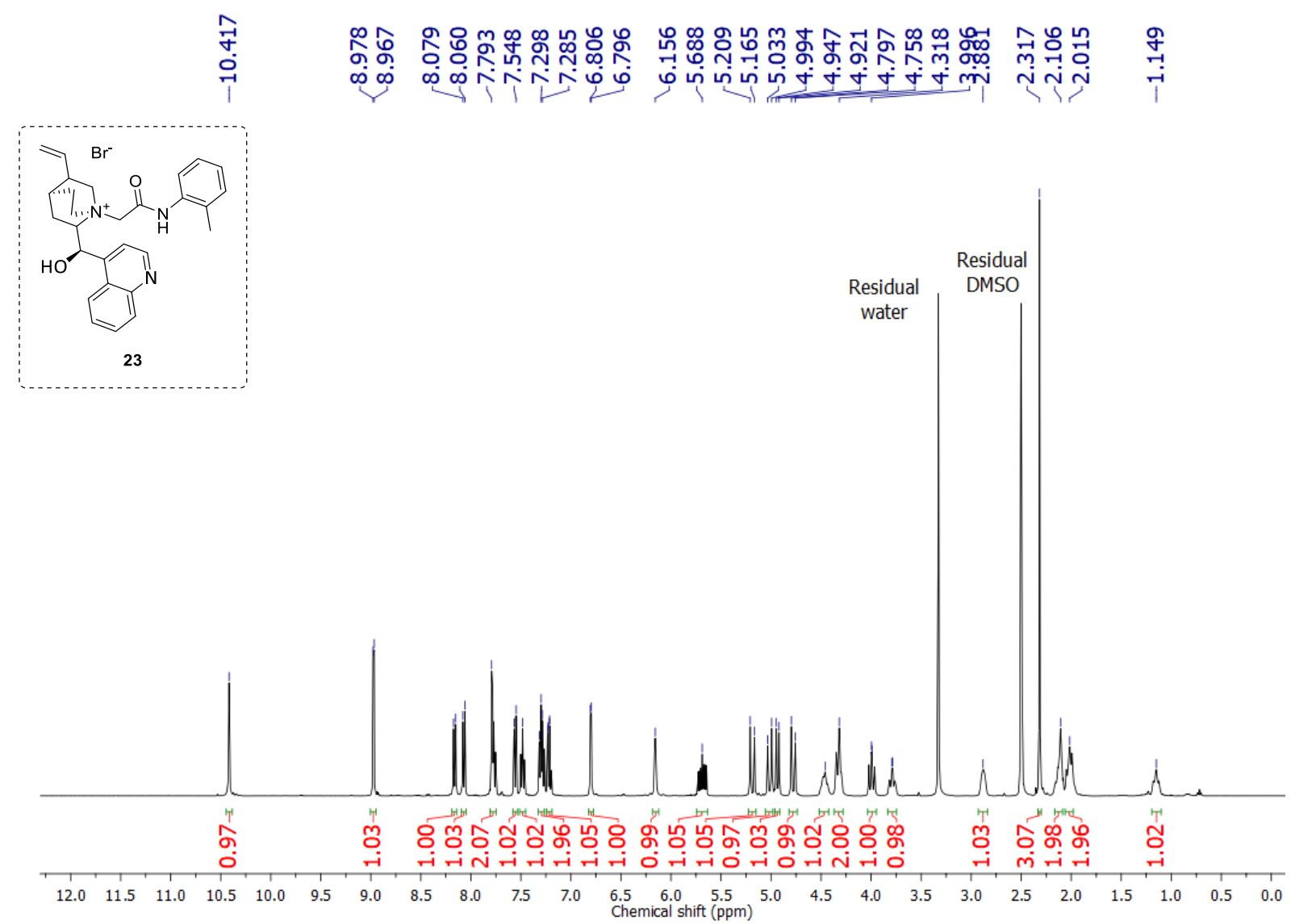

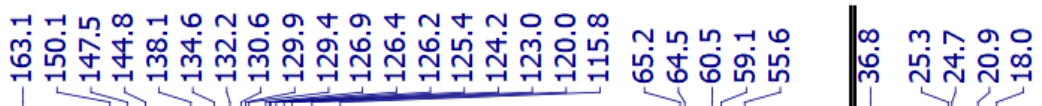

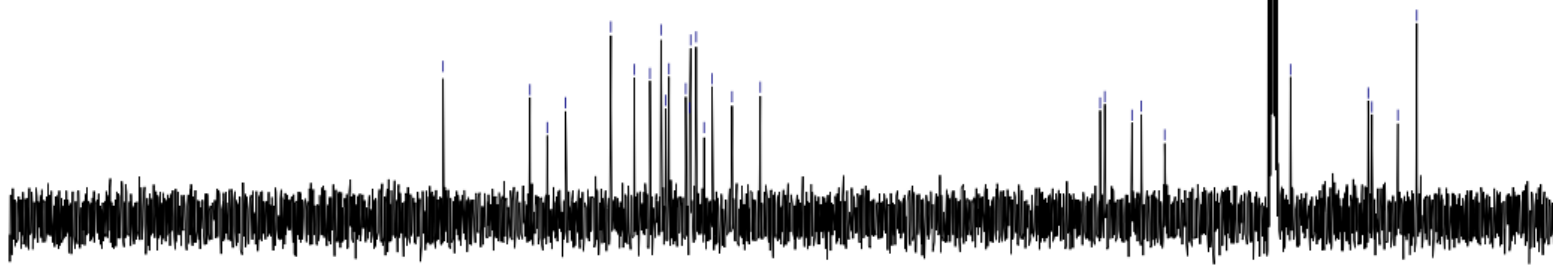

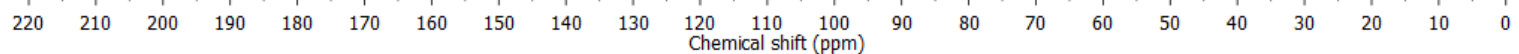

Fig. S5 ${ }^{1} \mathrm{H}$ NMR (400 MHz) and ${ }^{13} \mathrm{C}$ NMR (100 MHz) spectra of compound 23 in DMSO-d 6 . 

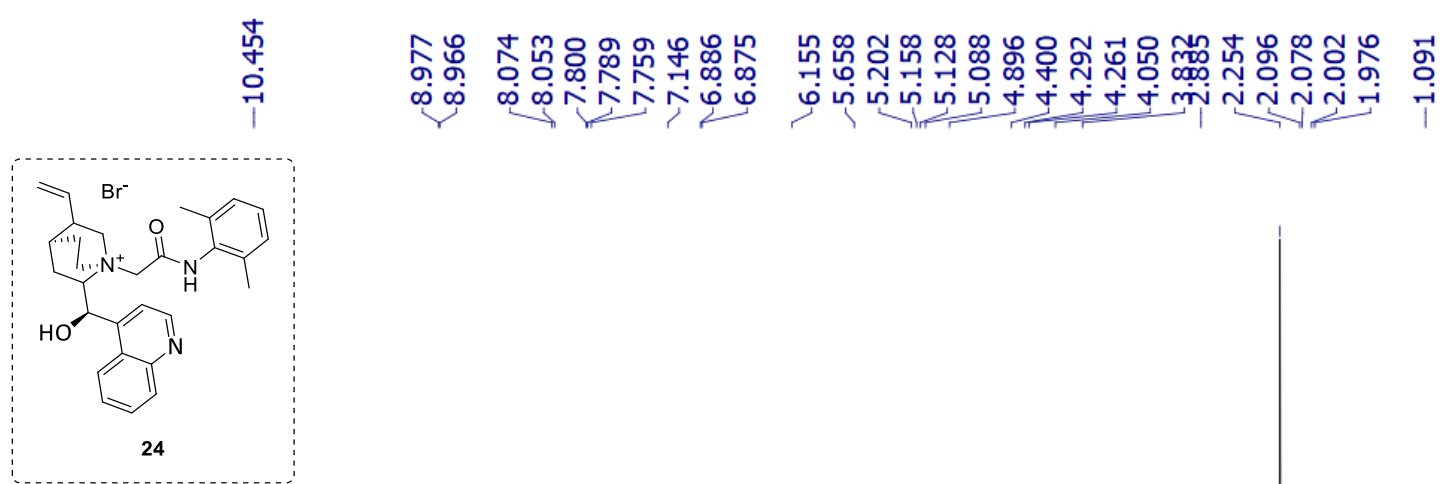

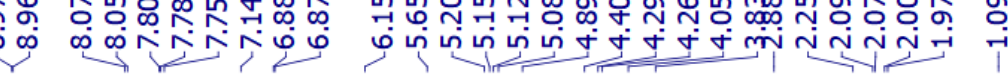
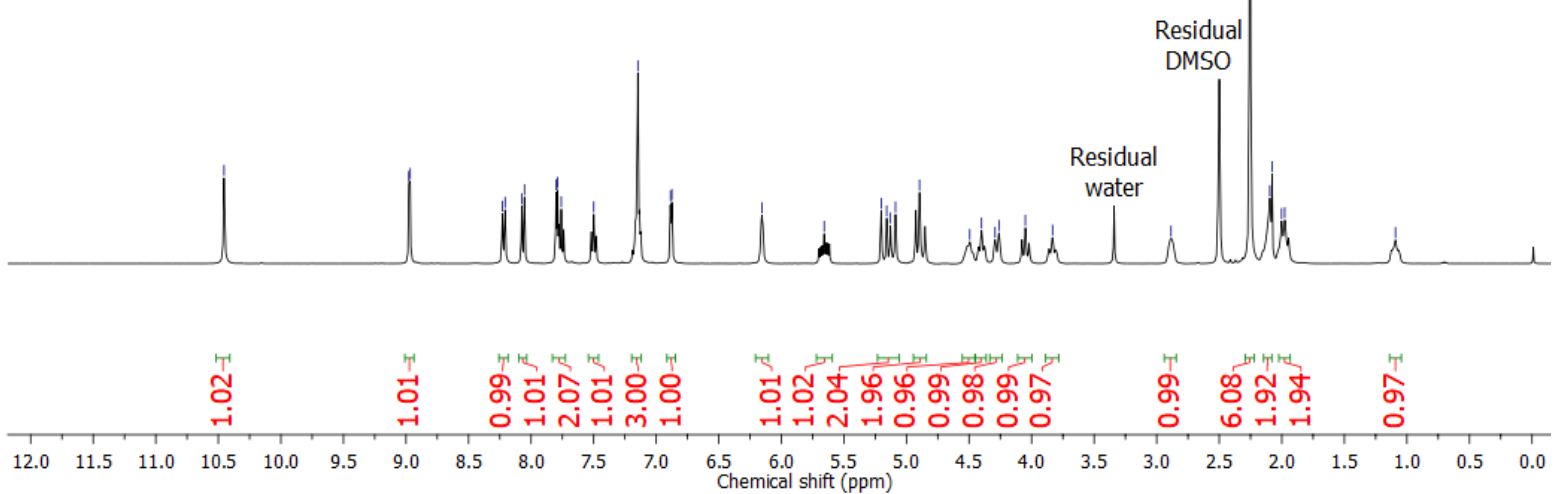

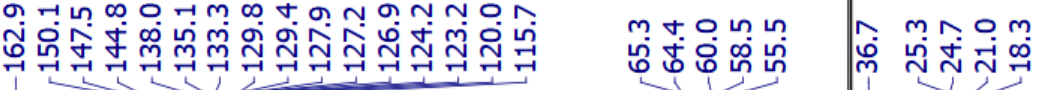

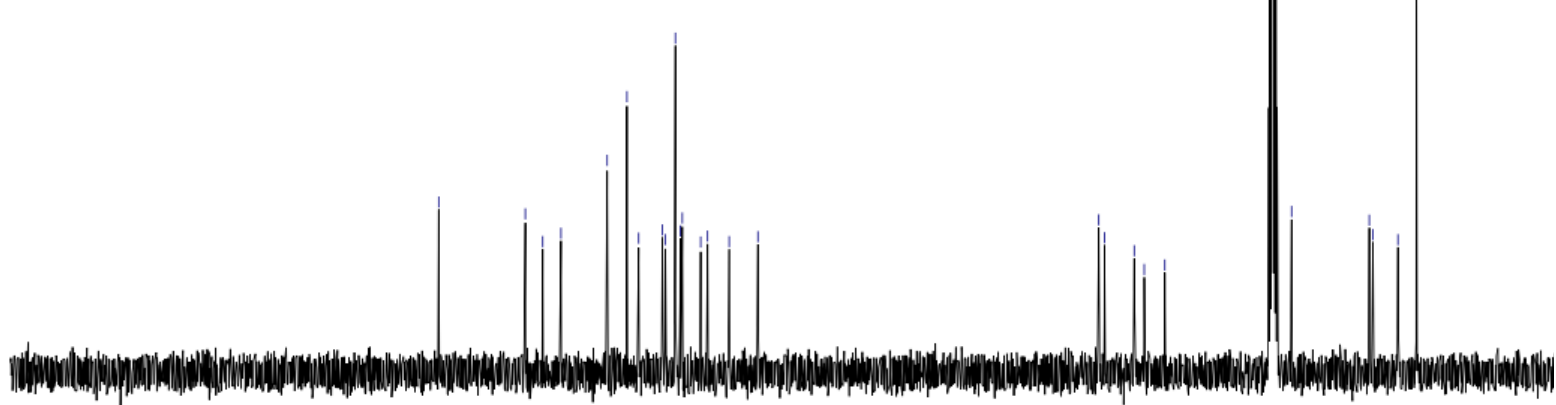

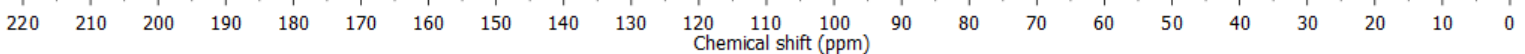

Fig. S6 ${ }^{1} \mathrm{H}$ NMR (400 MHz) and ${ }^{13} \mathrm{C}$ NMR (100 MHz) spectra of compound 24 in DMSO-d6. 

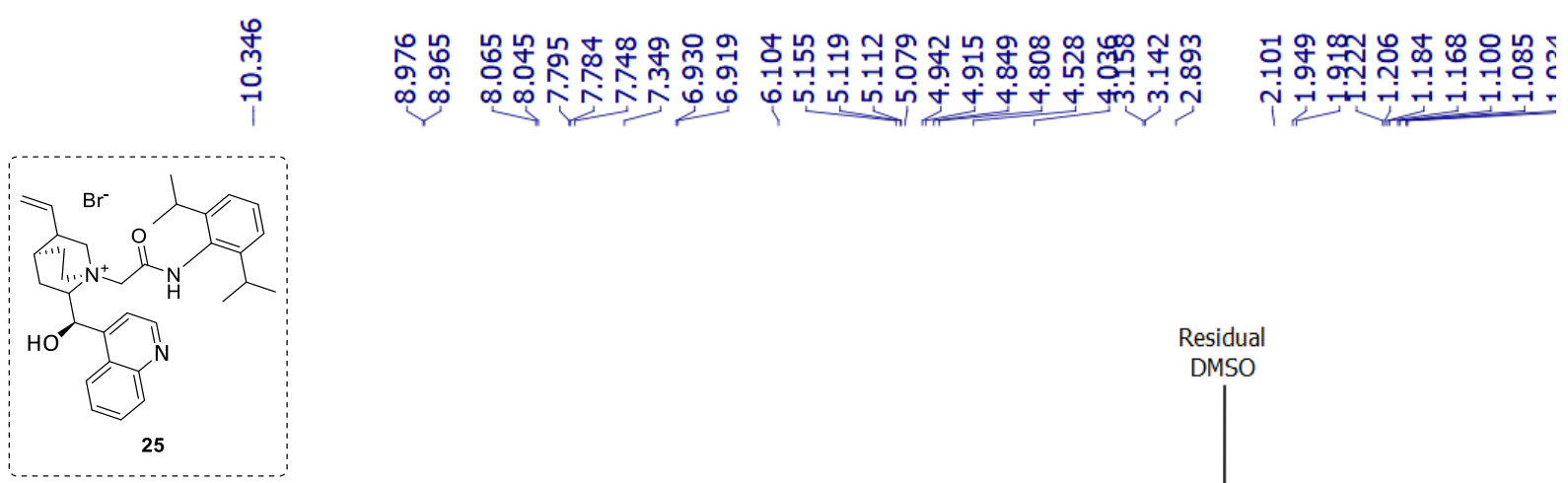

Residual DMSO

25

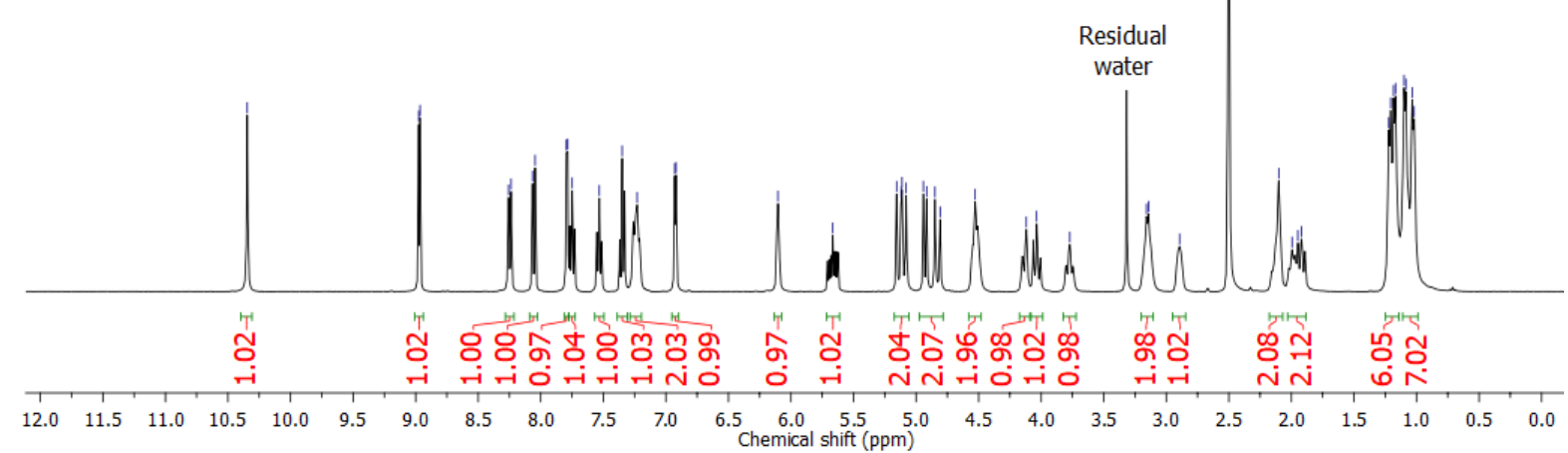

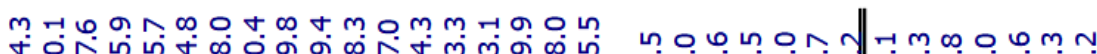

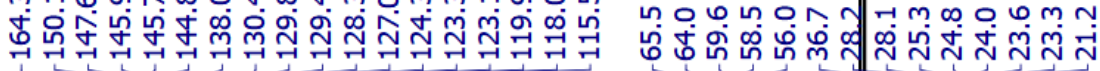
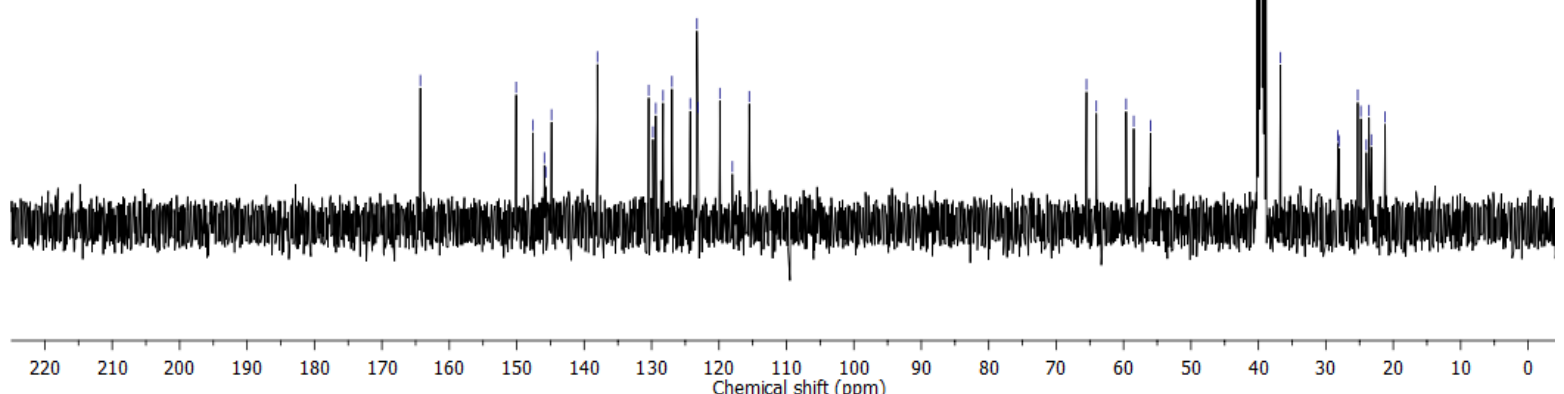

Fig. $57{ }^{1} \mathrm{H}$ NMR (400 MHz) and ${ }^{13} \mathrm{C}$ NMR (100 MHz) spectra of compound 25 in DMSO-d6. 


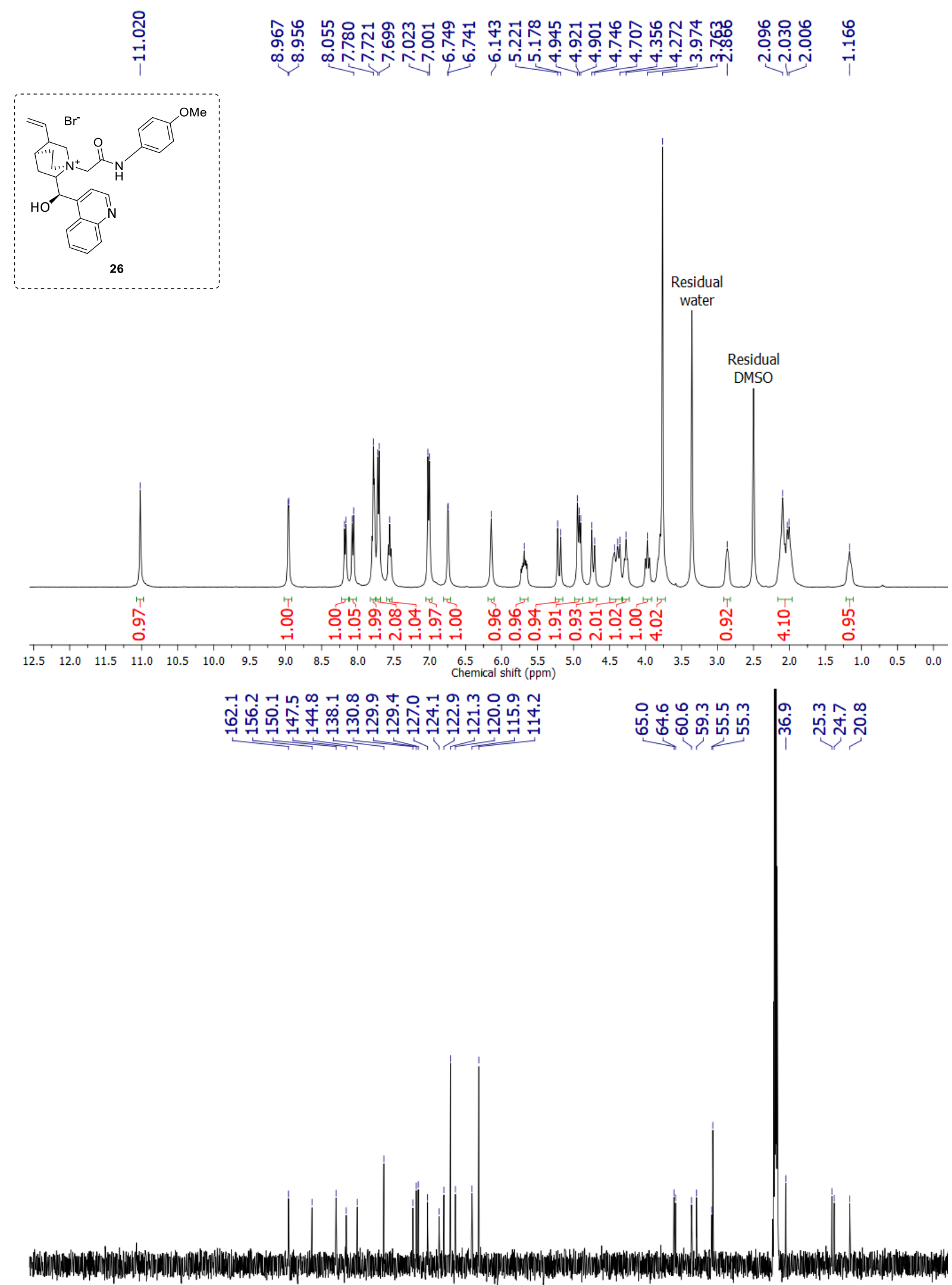

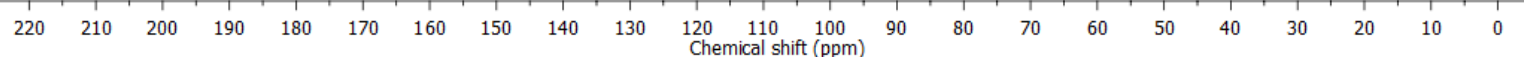

Fig. $58{ }^{1} \mathrm{H}$ NMR (400 MHz) and ${ }^{13} \mathrm{C}$ NMR (100 MHz) spectra of compound 26 in DMSO- $d_{6}$. 

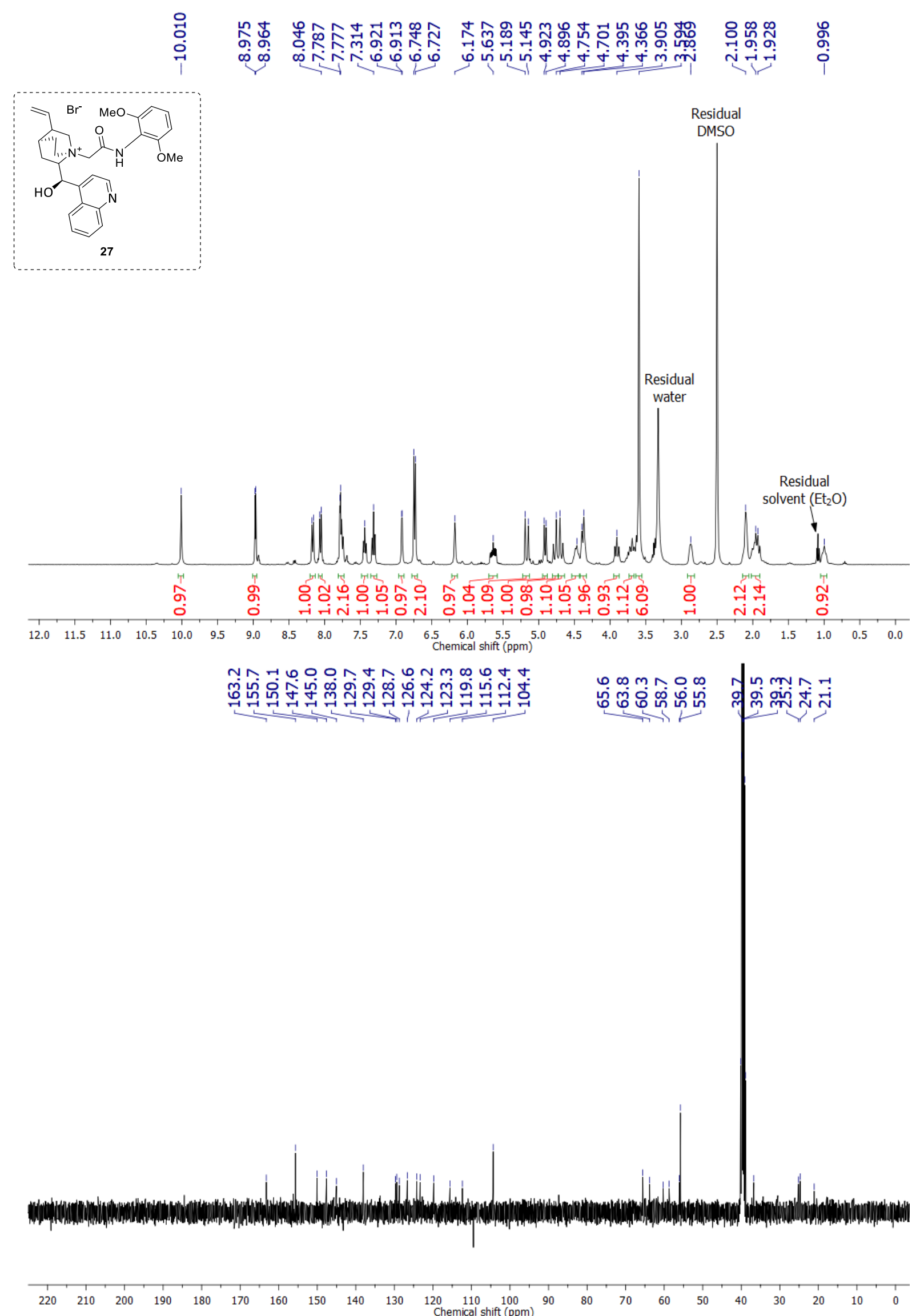

Fig. $59{ }^{1} \mathrm{H}$ NMR (400 MHz) and ${ }^{13} \mathrm{C}$ NMR (100 MHz) spectra of compound 27 in DMSO- $d_{6}$. 


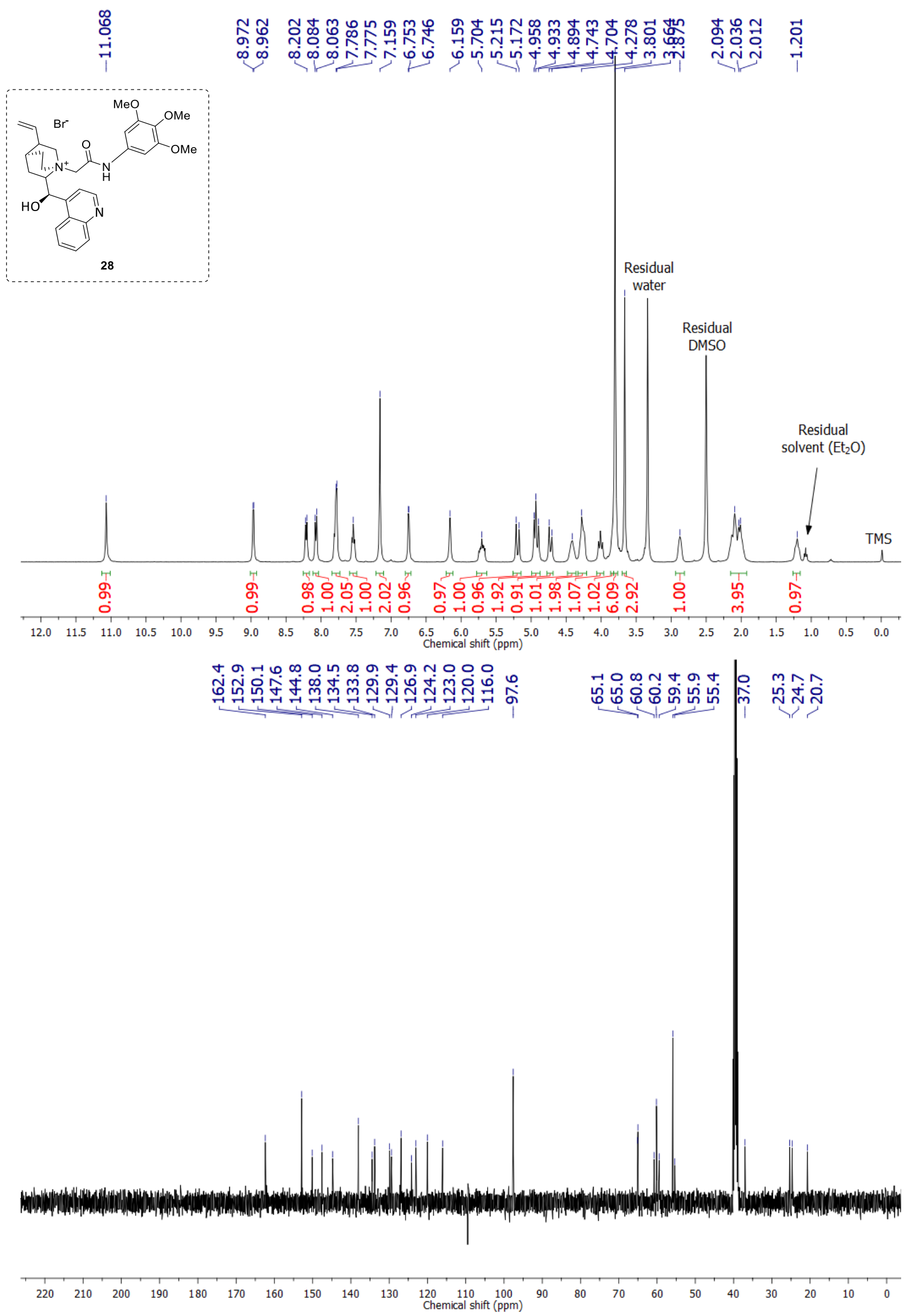

Fig. $\mathbf{S 1 0}{ }^{1} \mathrm{H}$ NMR (400 MHz) and ${ }^{13} \mathrm{C}$ NMR (100 MHz) spectra of compound 28 in DMSO- $d_{6}$. 

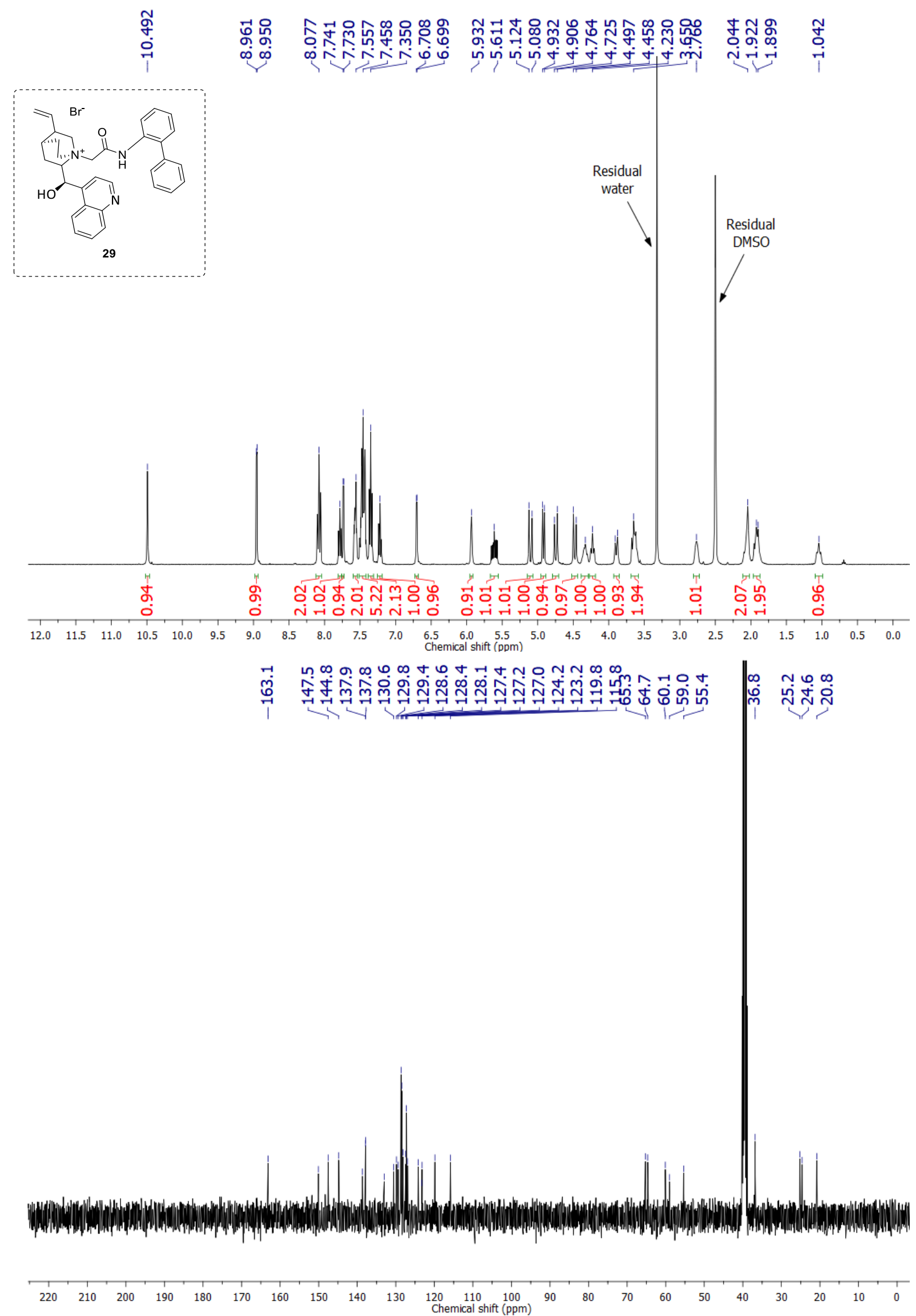

Fig. S11 ${ }^{1} \mathrm{H}$ NMR (400 MHz) and ${ }^{13} \mathrm{C}$ NMR (100 MHz) spectra of compound 29 in DMSO- $d_{6}$. 


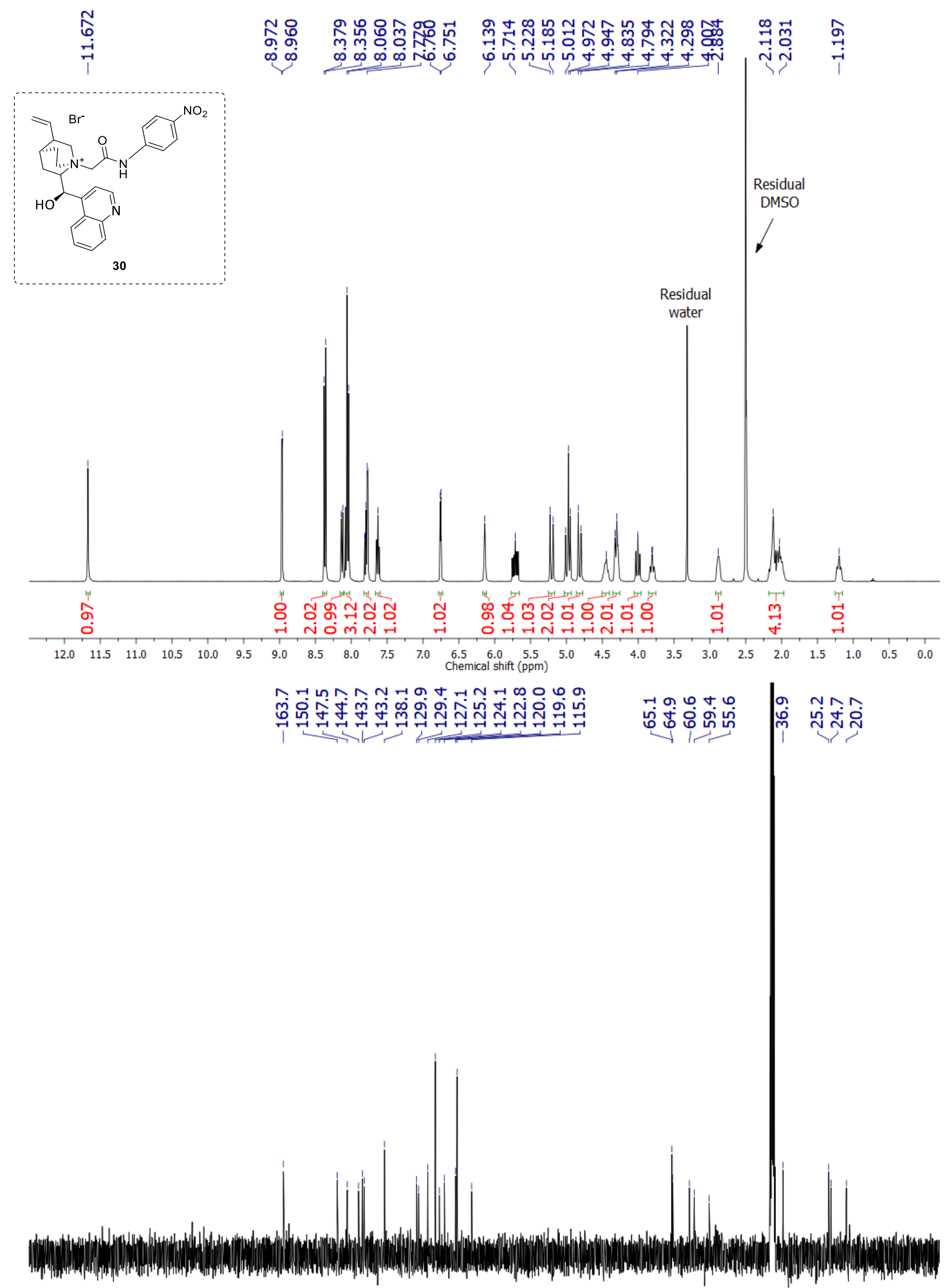

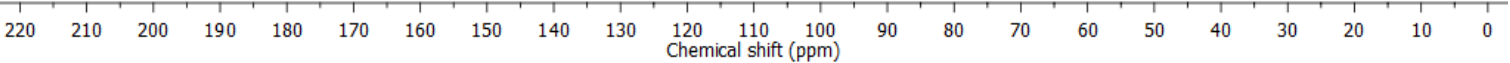

Fig. S12 ${ }^{1} \mathrm{H}$ NMR (400 MHz) and ${ }^{13} \mathrm{C}$ NMR (100 MHz) spectra of compound $\mathbf{3 0}$ in DMSO- $d_{6}$. 


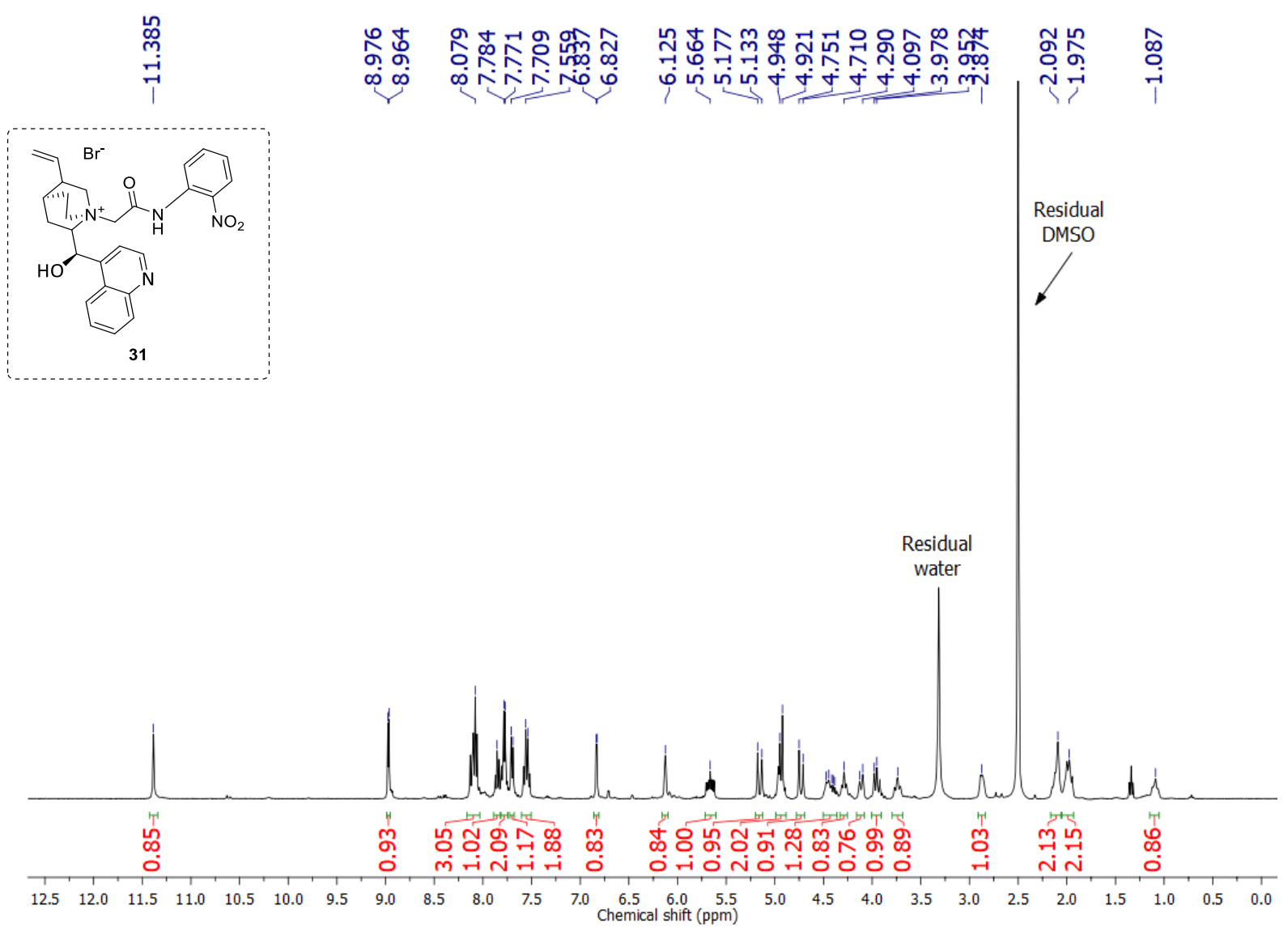

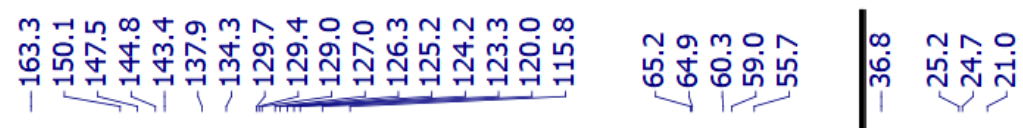
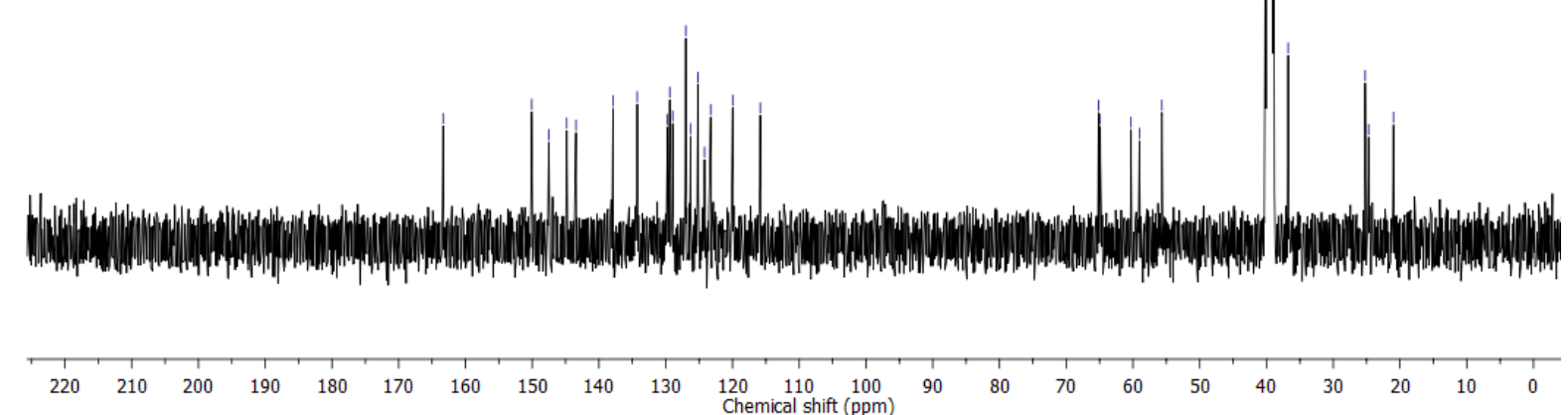

Fig. S13 ${ }^{1} \mathrm{H}$ NMR (400 MHz) and ${ }^{13} \mathrm{C}$ NMR (100 MHz) spectra of compound 31 in DMSO- $d_{6}$. 


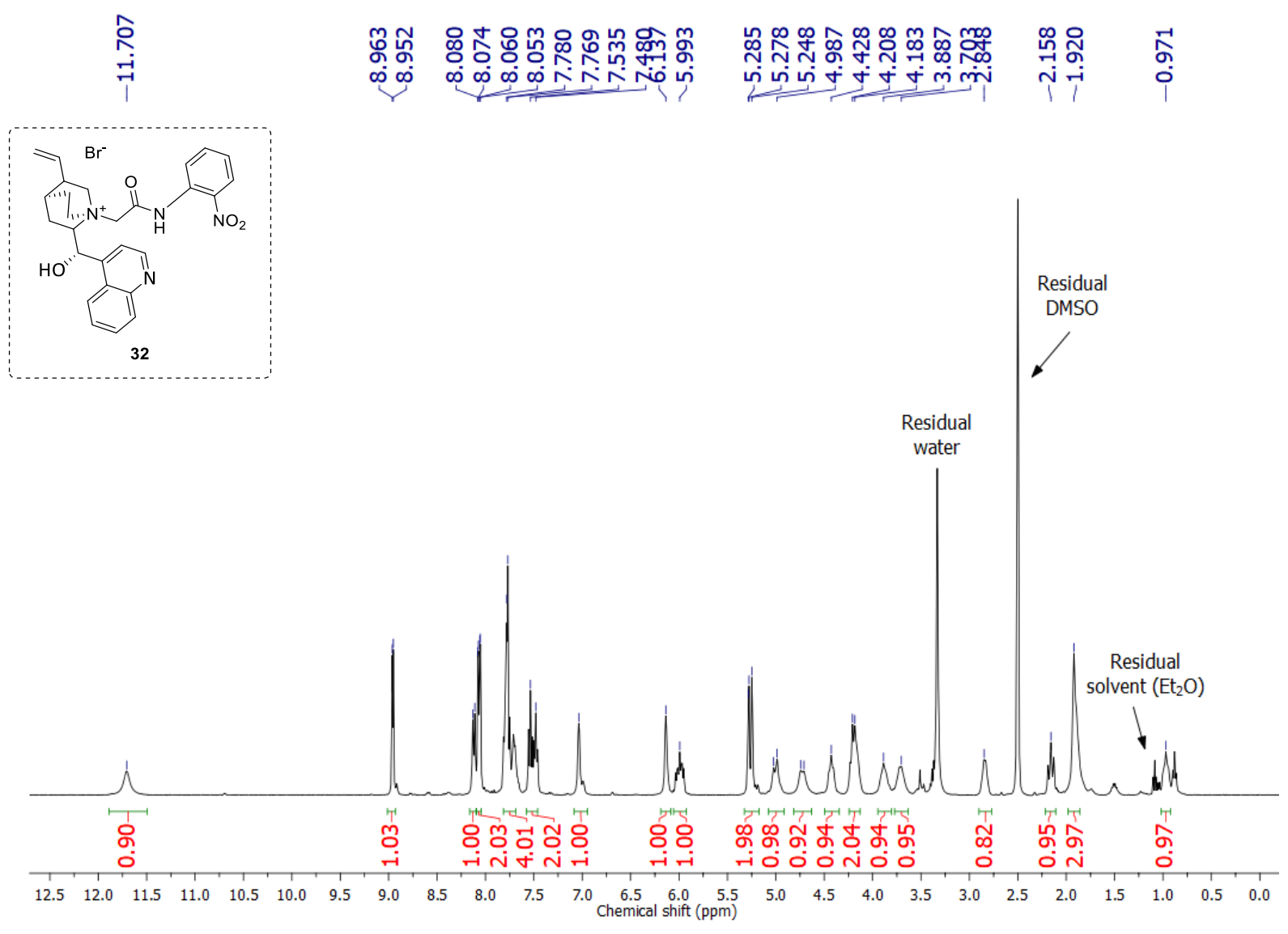

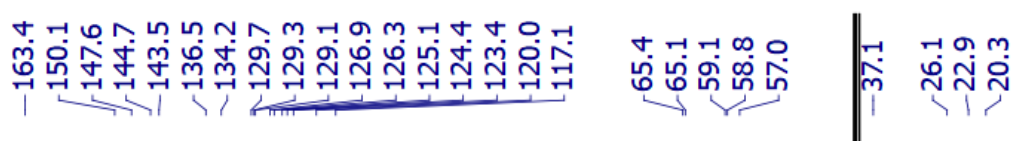

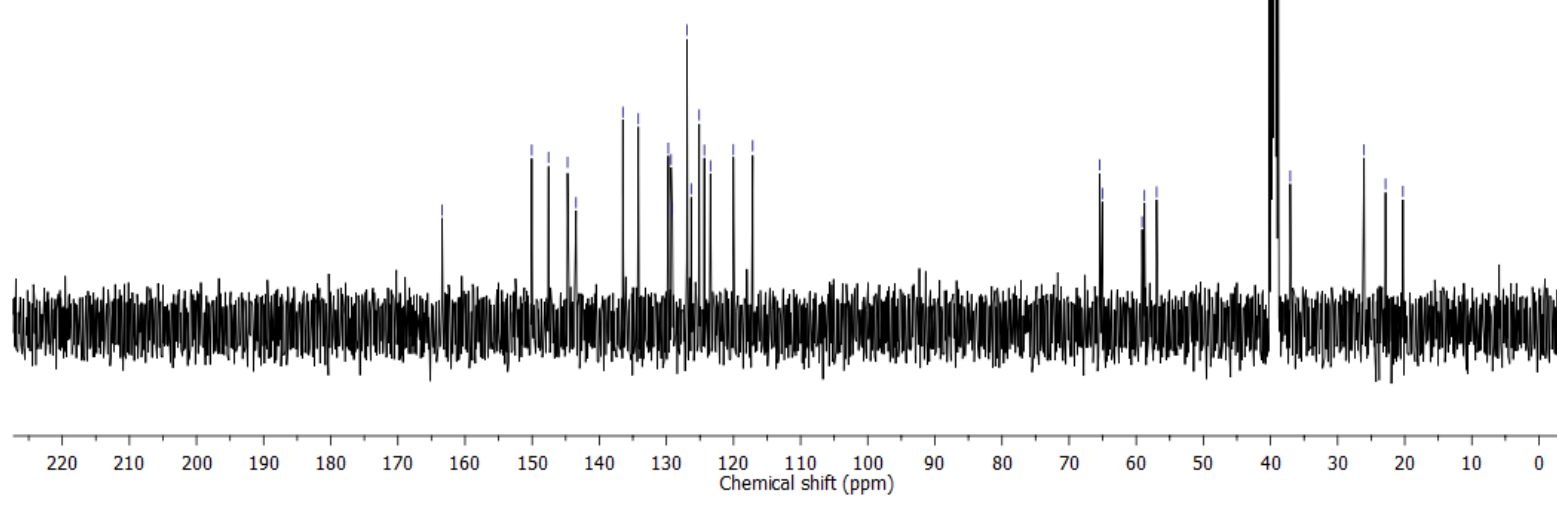

Fig. S14 ${ }^{1} \mathrm{H}$ NMR (400 MHz) and ${ }^{13} \mathrm{C}$ NMR (100 MHz) spectra of compound 32 in DMSO- $d_{6}$. 

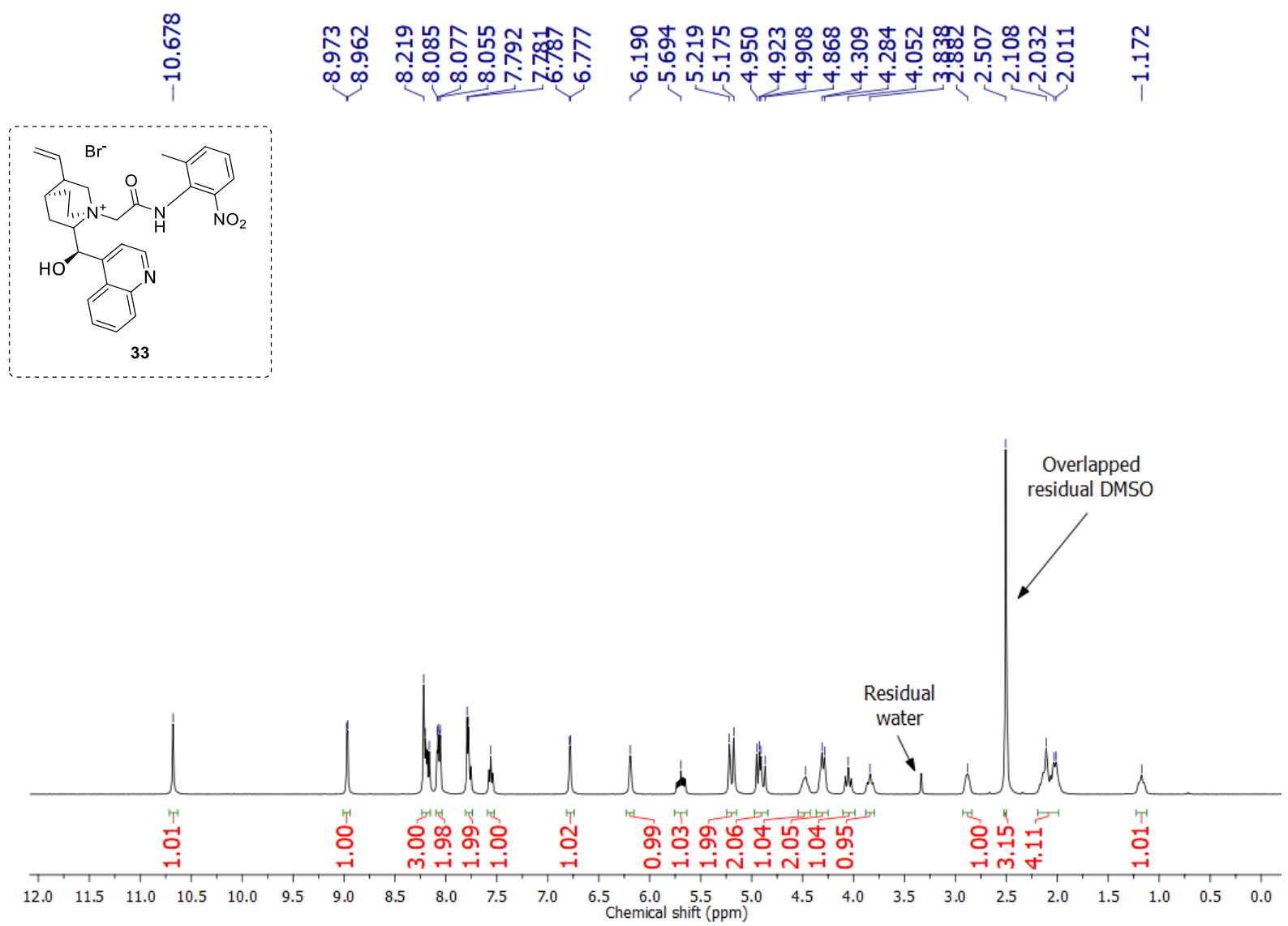

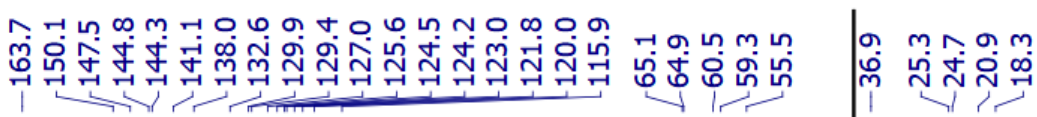

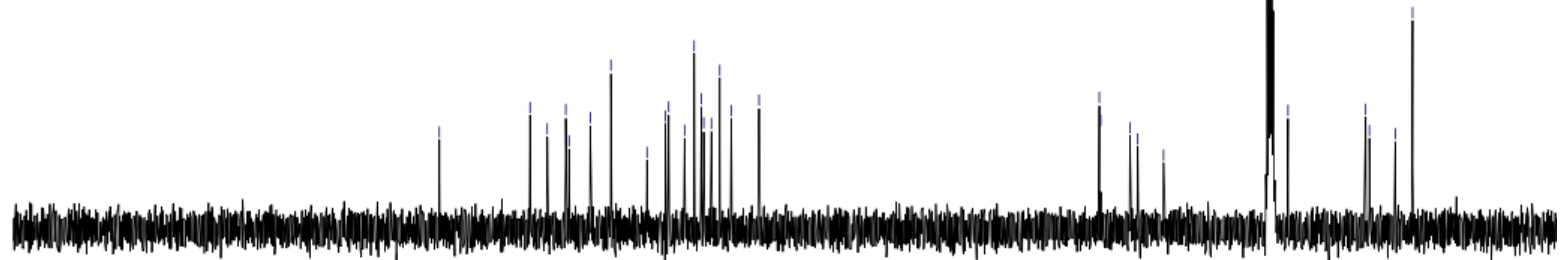

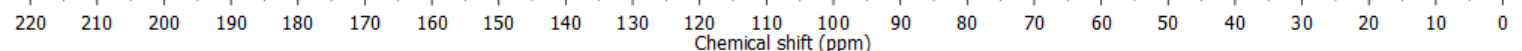

Fig. S15 ${ }^{1} \mathrm{H}$ NMR $(400 \mathrm{MHz})$ and ${ }^{13} \mathrm{C}$ NMR $(100 \mathrm{MHz})$ spectra of compound 33 in DMSO-d6. 


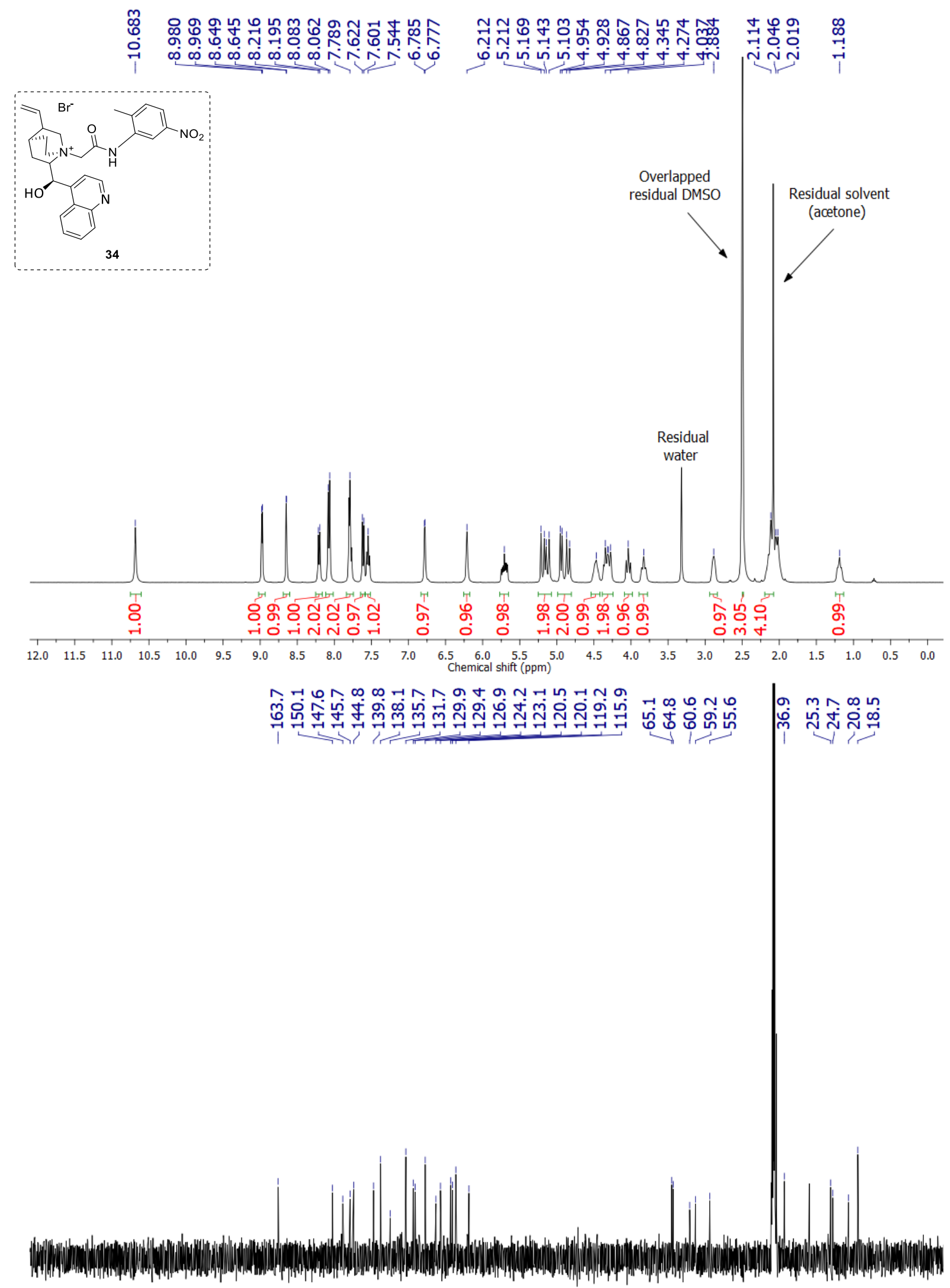

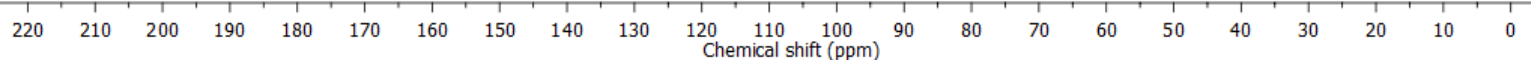

Fig. S16 ${ }^{1} \mathrm{H}$ NMR (400 MHz) and ${ }^{13} \mathrm{C}$ NMR (100 MHz) spectra of compound 34 in DMSO- $d_{6}$. 


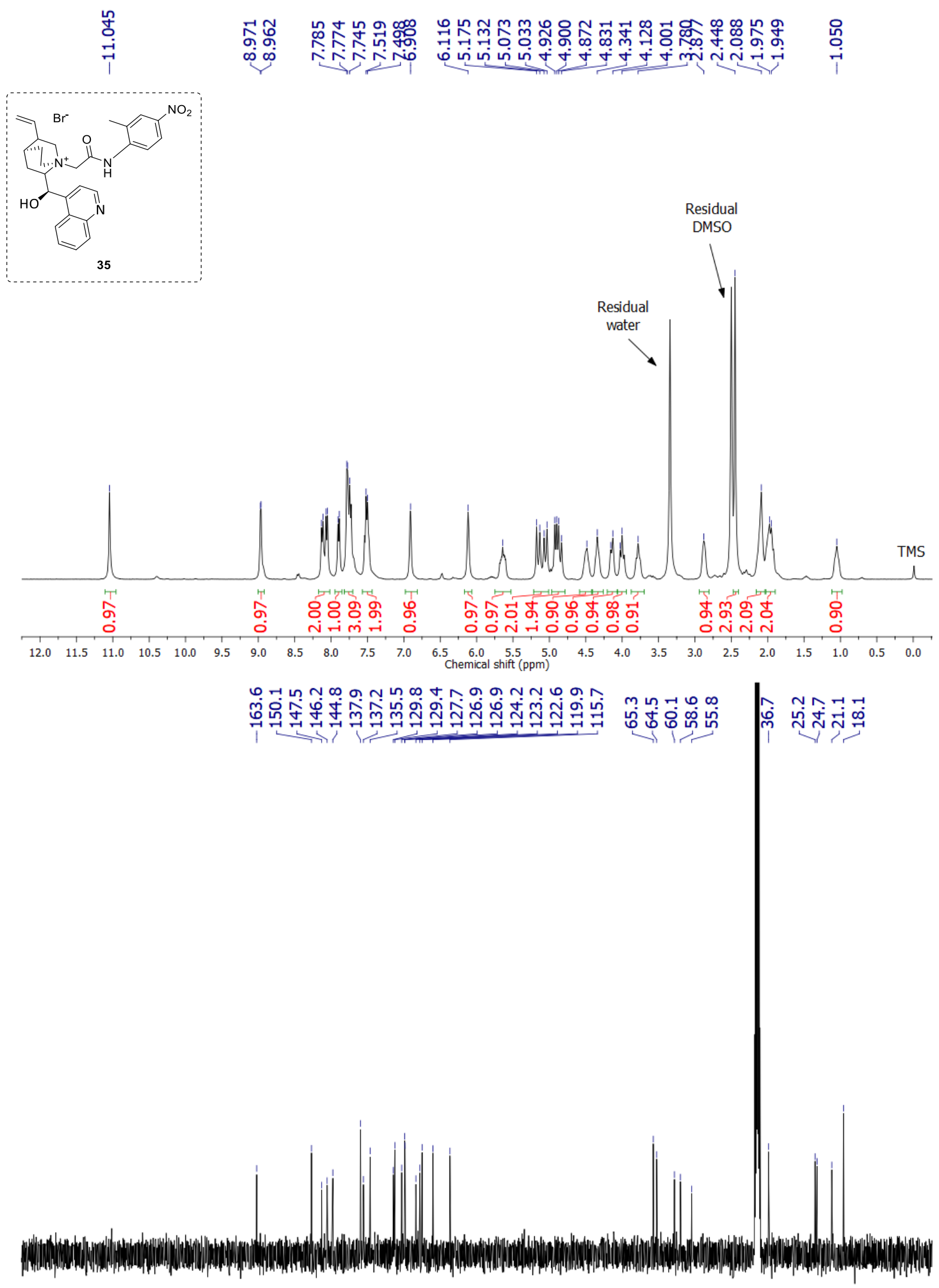

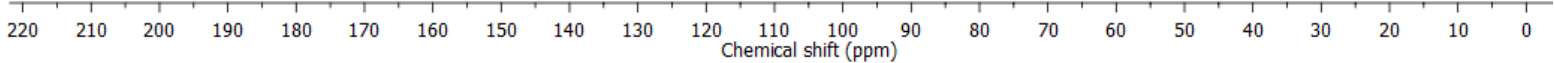

Fig. S17 ${ }^{1} \mathrm{H}$ NMR (400 MHz) and ${ }^{13} \mathrm{C}$ NMR (100 MHz) spectra of compound 35 in DMSO- $d_{6}$. 


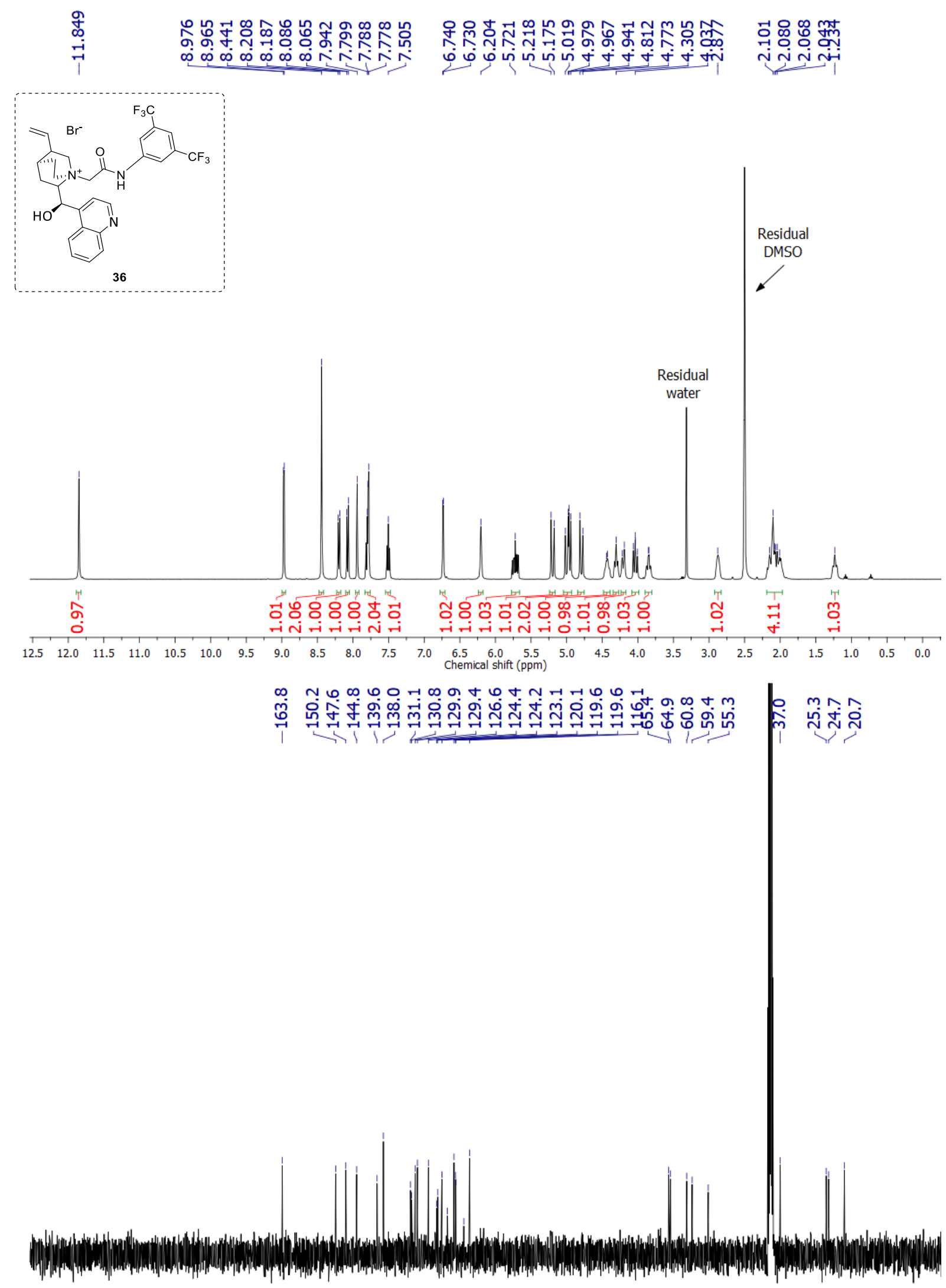

$\begin{array}{llllllllllllllllllllllllllllll}220 & 210 & 200 & 190 & 180 & 170 & 160 & 150 & 140 & 130 & 120 & 110 & 100 & 90 & 80 & 70 & 60 & 50 & 40 & 30 & 20 & 10 & 0\end{array}$

Fig. S18 ${ }^{1} \mathrm{H}$ NMR (400 MHz) and ${ }^{13} \mathrm{C}$ NMR (100 MHz) spectra of compound 36 in DMSO-d 6 . 


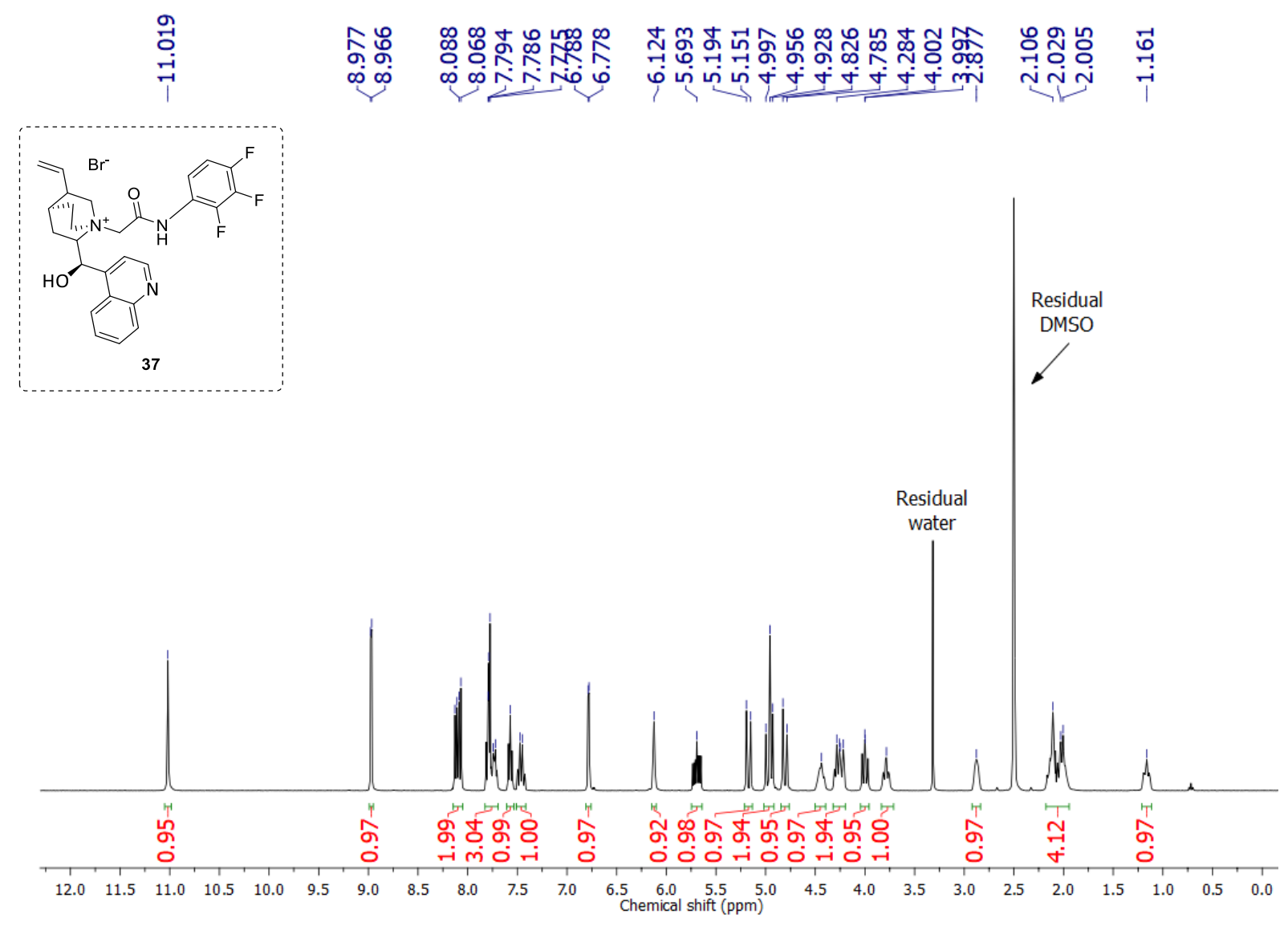

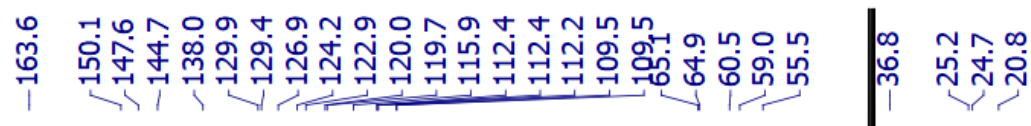

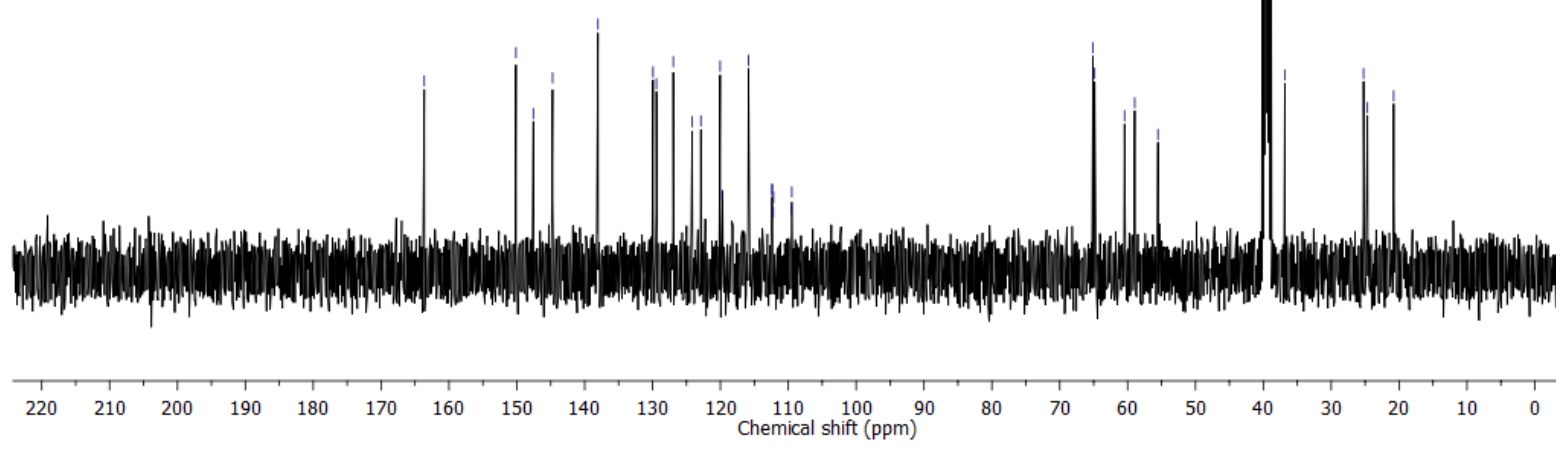

Fig. S19 ${ }^{1} \mathrm{H}$ NMR (400 MHz) and ${ }^{13} \mathrm{C}$ NMR (100 MHz) spectra of compound 37 in DMSO- $d_{6}$. 


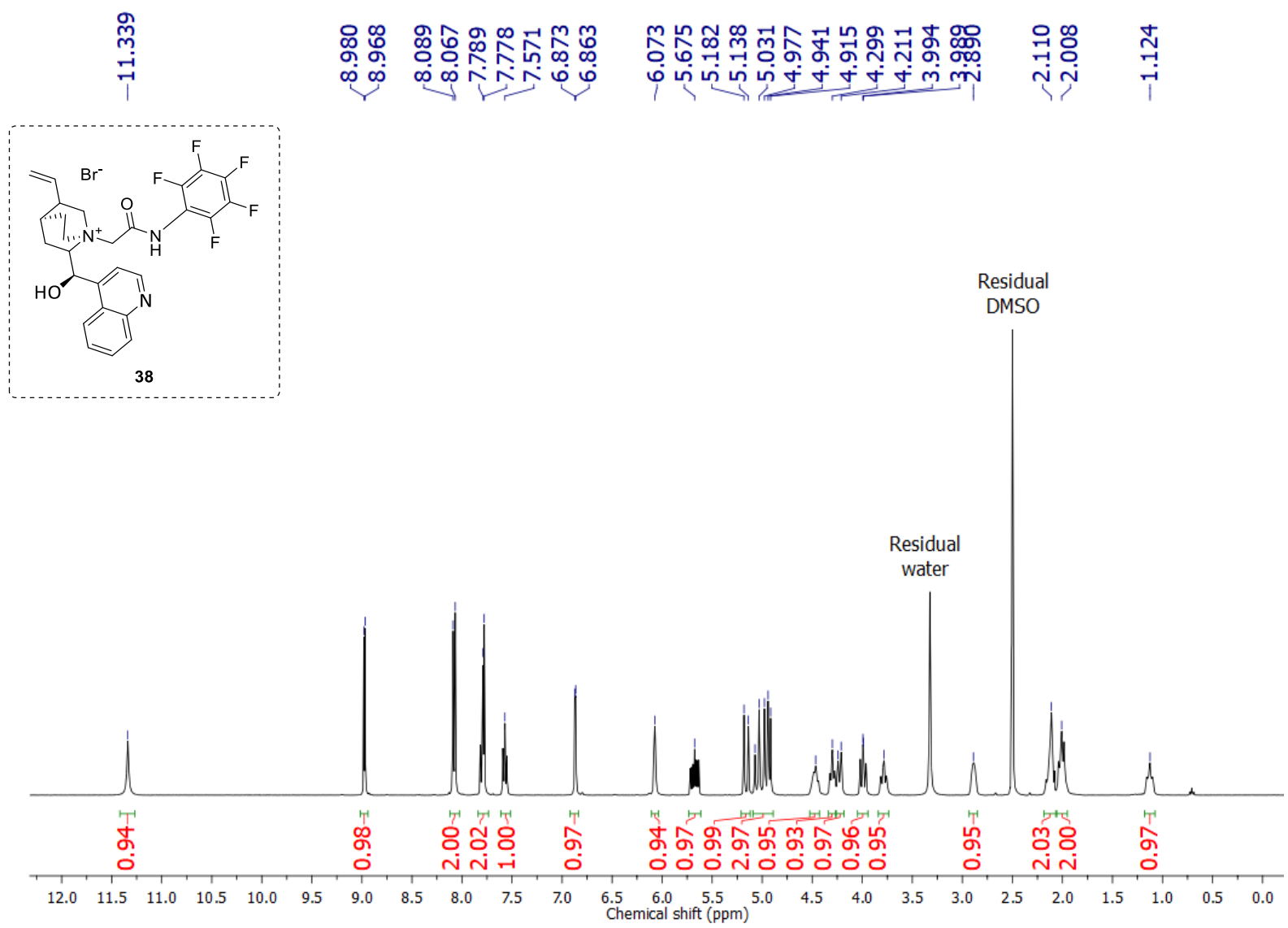

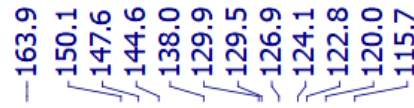

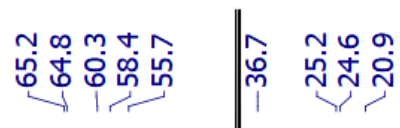

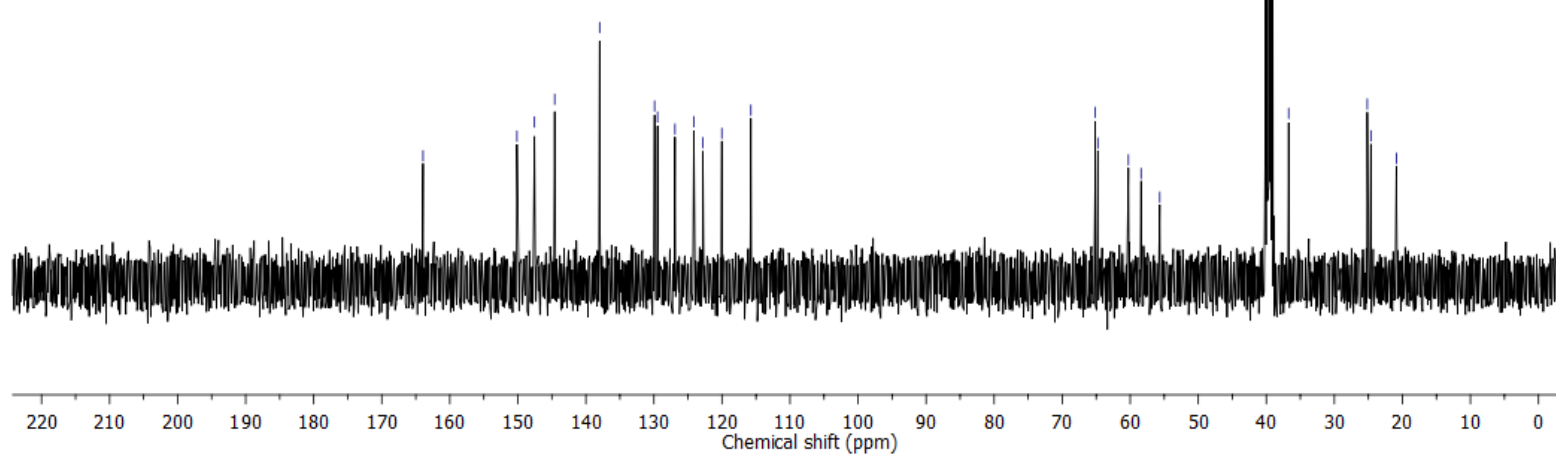

Fig. S20 ${ }^{1} \mathrm{H}$ NMR (400 MHz) and ${ }^{13} \mathrm{C}$ NMR (100 MHz) spectra of compound 38 in DMSO- $d_{6}$. 


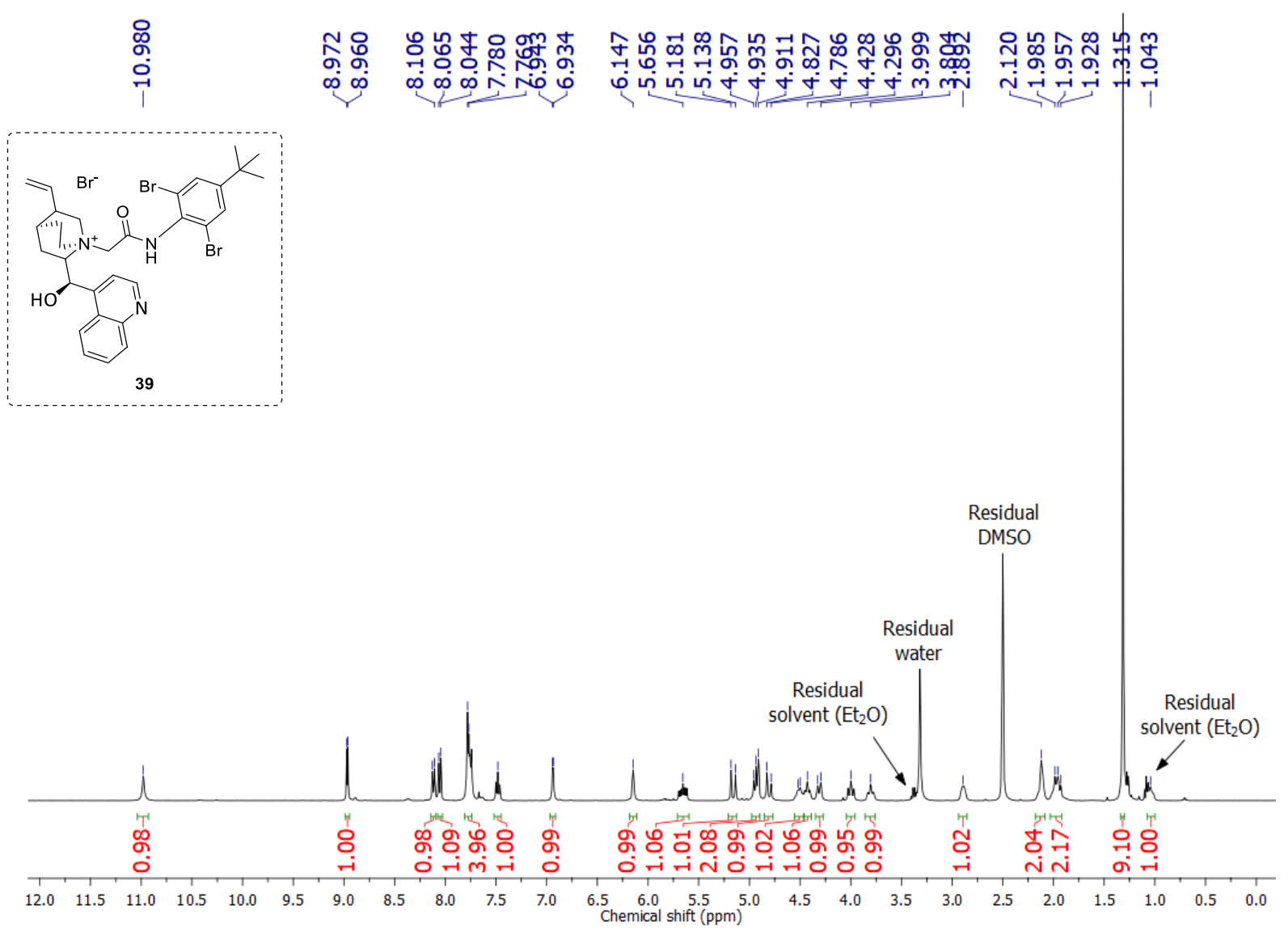

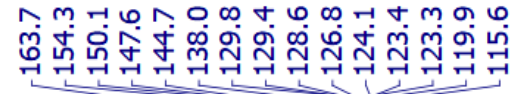

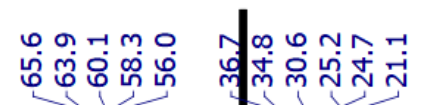

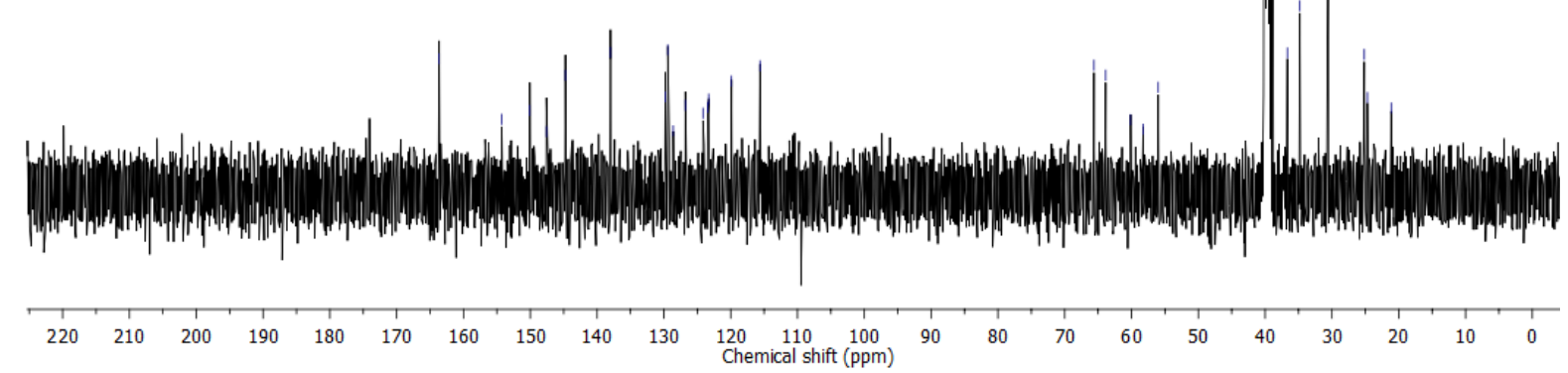

Fig. S21 ${ }^{1} \mathrm{H}$ NMR (400 MHz) and ${ }^{13} \mathrm{C}$ NMR (100 MHz) spectra of compound 39 in DMSO- $d_{6}$. 

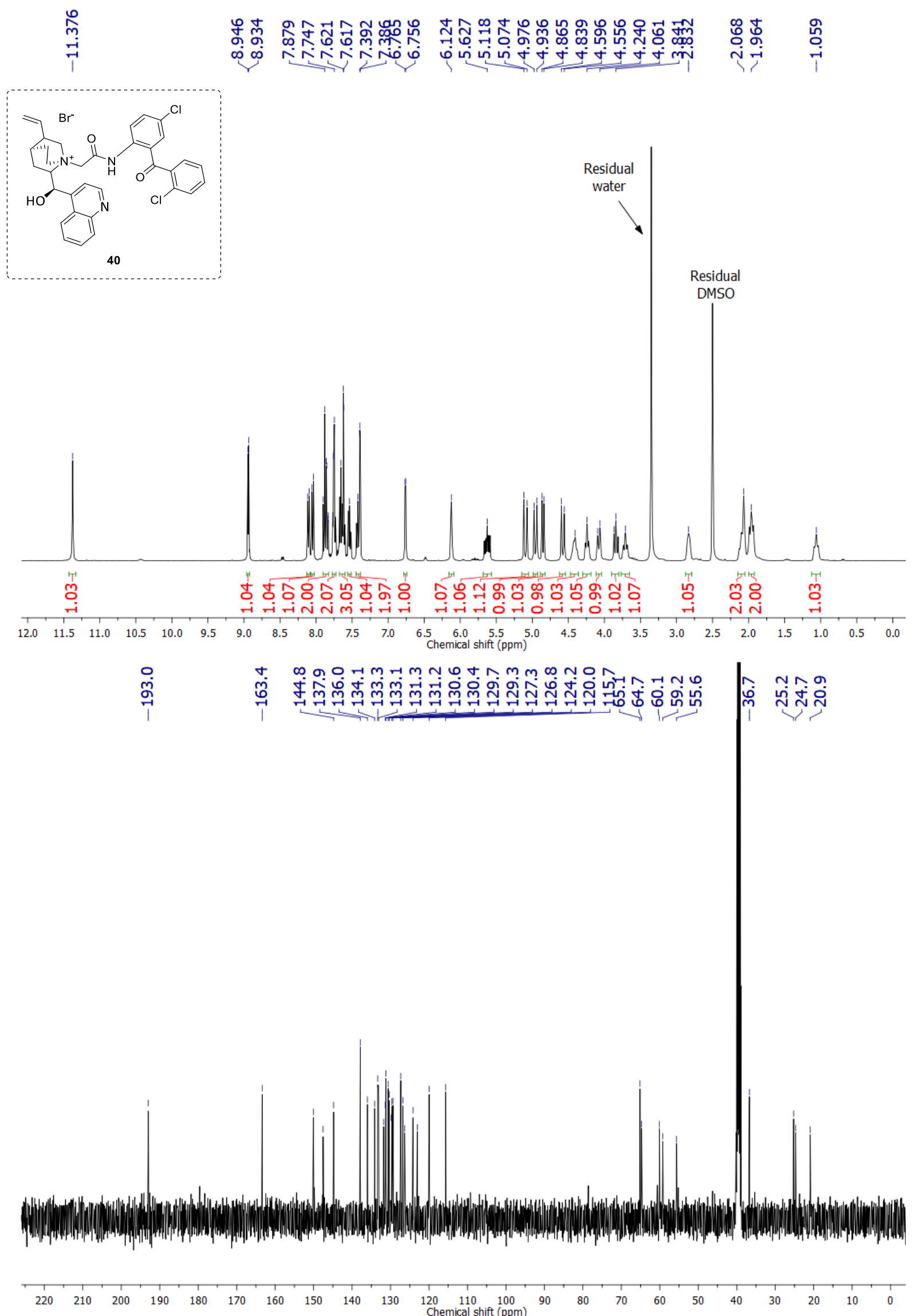

Fig. S22 ${ }^{1} \mathrm{H}$ NMR (400 MHz) and ${ }^{13} \mathrm{C}$ NMR (100 MHz) spectra of compound 40 in DMSO-d6. 


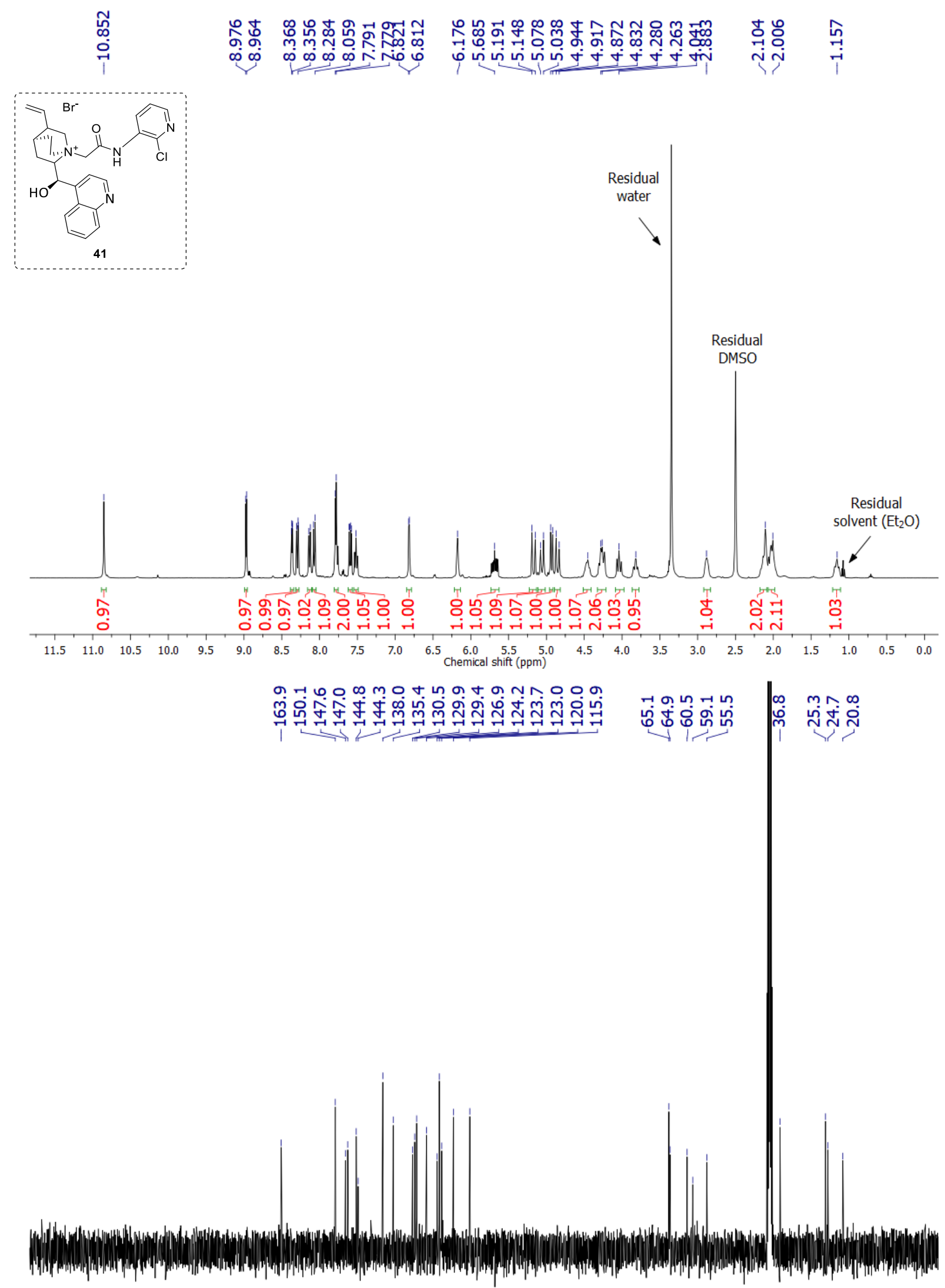

$\begin{array}{llllllllllllllllllllllll}220 & 210 & 200 & 190 & 180 & 170 & 160 & 150 & 140 & 130 & 120 & 110 & 100 & 90 & 80 & 70 & 60 & 50 & 40 & 30 & 20 & 10 & 0\end{array}$

Fig. S23 ${ }^{1} \mathrm{H}$ NMR (400 MHz) and ${ }^{13} \mathrm{C}$ NMR (100 MHz) spectra of compound 41 in DMSO- $d_{6}$. 


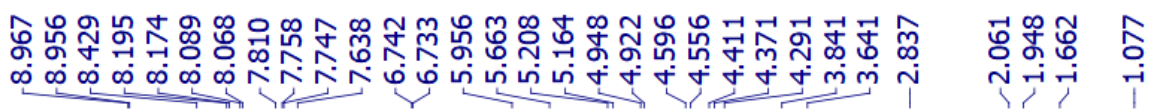
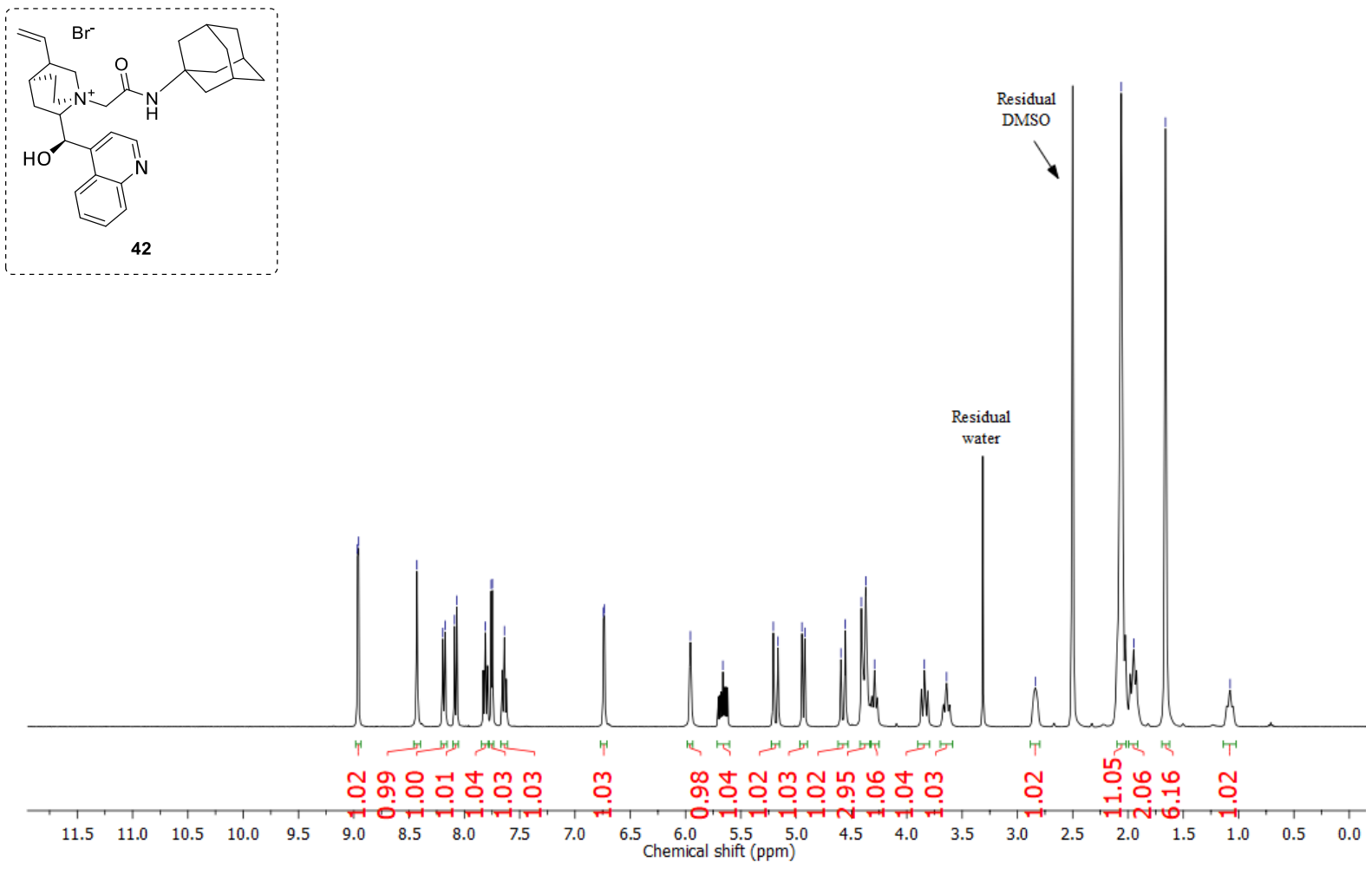

mำ 뚜

ஸூ่

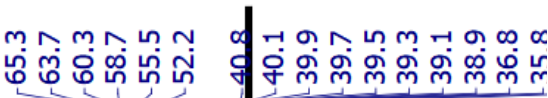

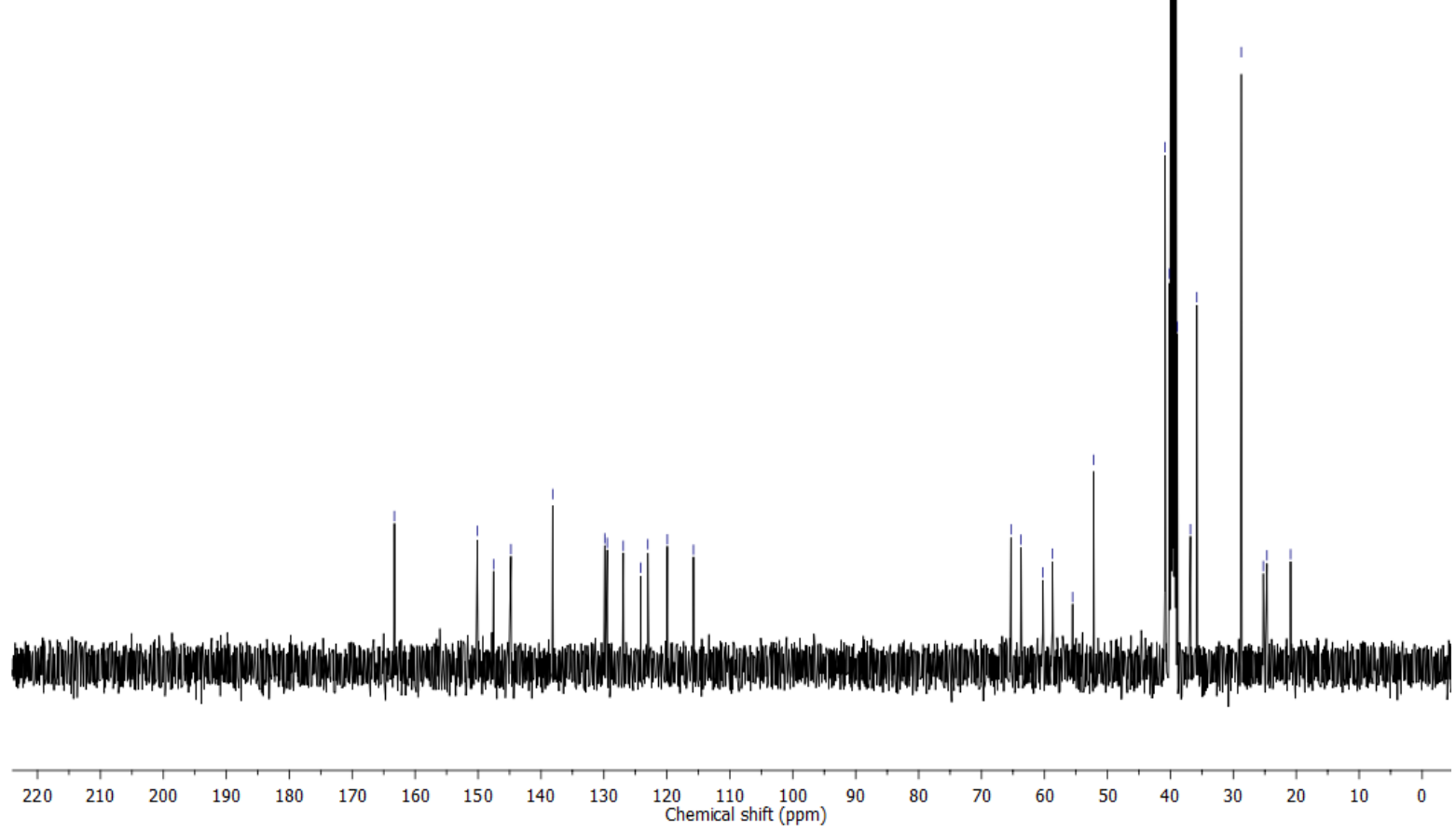

Fig. S24 ${ }^{1} \mathrm{H}$ NMR (400 MHz) and ${ }^{13} \mathrm{C}$ NMR (100 MHz) spectra of compound 42 in DMSO-d $d_{6}$. 


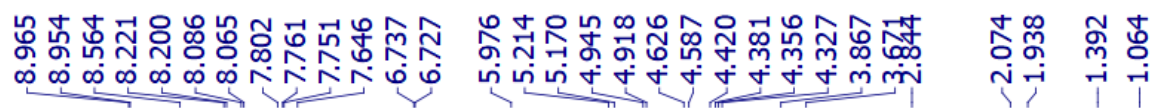
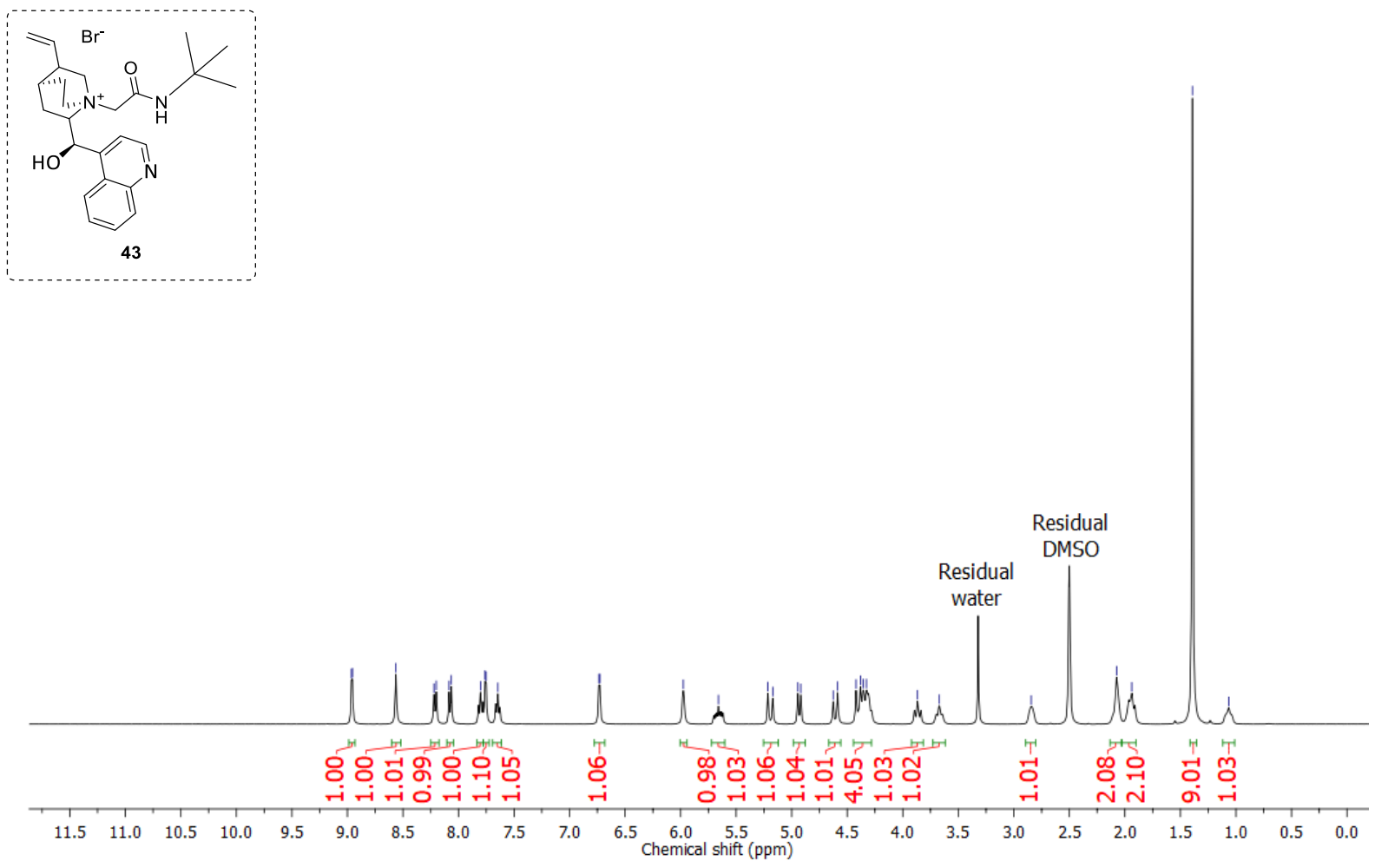

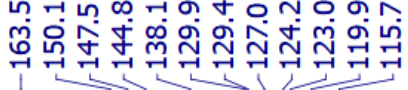

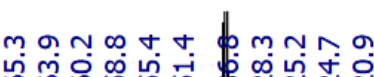

นูํํํํำ นึ่

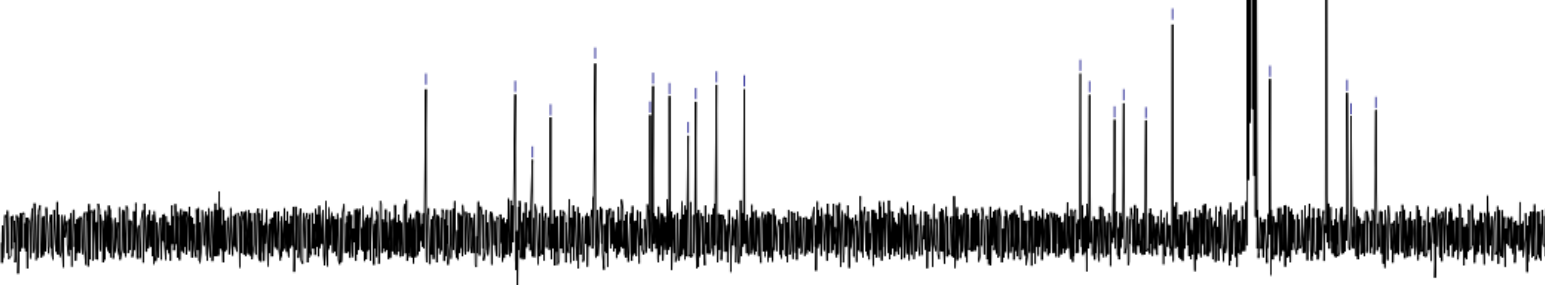

$\begin{array}{llllllllllllllllllllllll}220 & 210 & 200 & 190 & 180 & 170 & 160 & 150 & 140 & 130 & \begin{array}{c}120 \\ \text { Chemical shift }(\mathrm{ppm})\end{array} & 90 & 80 & 70 & 60 & 50 & 40 & 30 & 20 & 10 & 0\end{array}$

Fig. S25 ${ }^{1} \mathrm{H}$ NMR (400 MHz) and ${ }^{13} \mathrm{C}$ NMR (100 MHz) spectra of compound 43 in DMSO- $d_{6}$. 

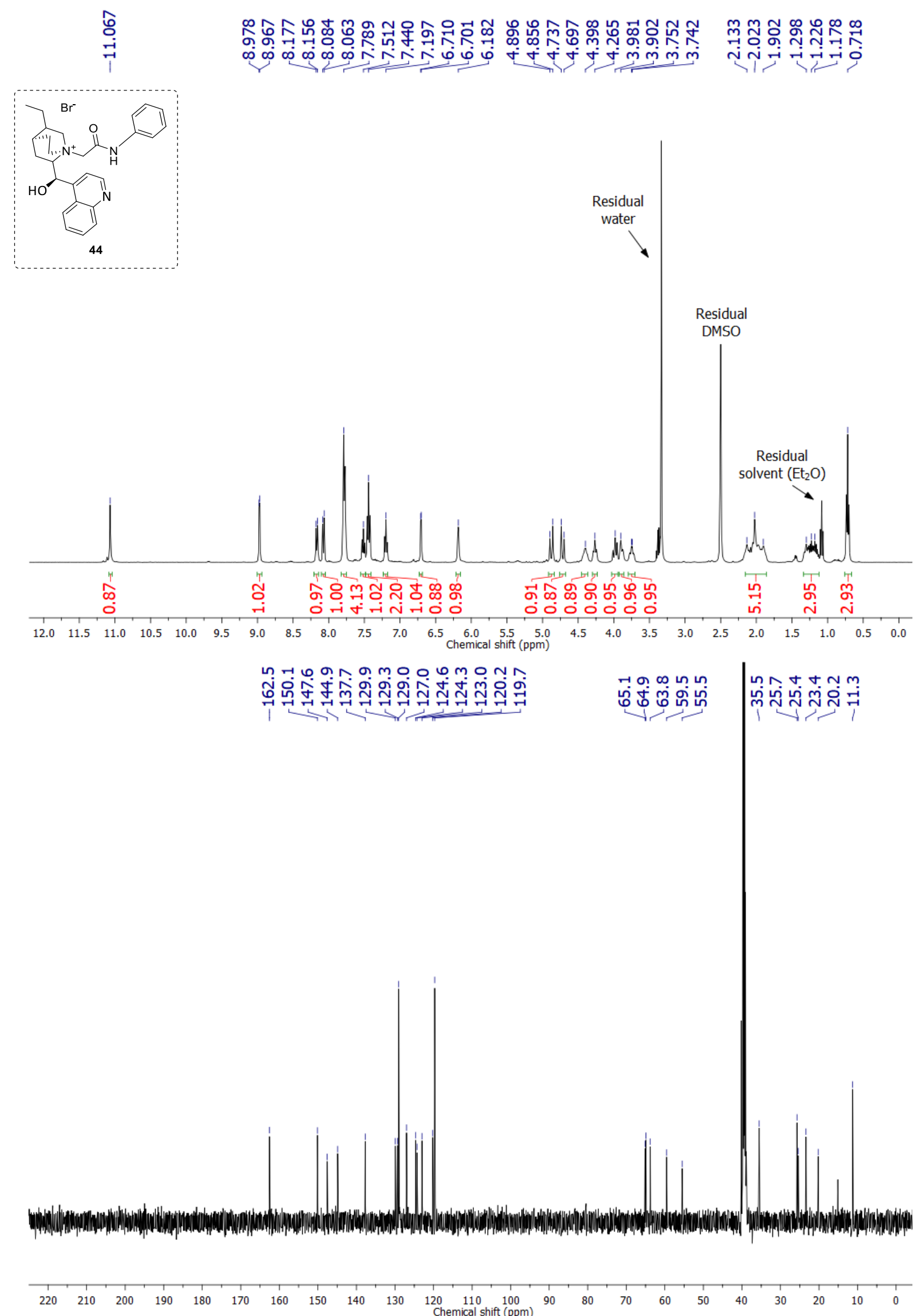

Fig. S26 ${ }^{1} \mathrm{H}$ NMR (400 MHz) and ${ }^{13} \mathrm{C}$ NMR (100 MHz) spectra of compound 44 in DMSO- $d_{6}$. 

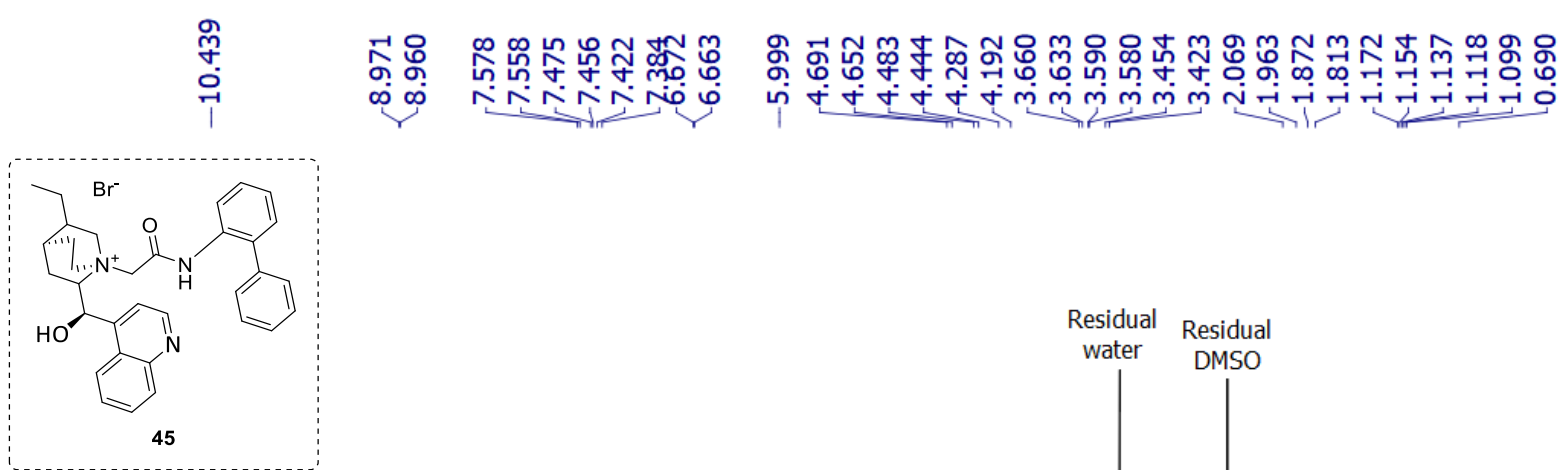

ம்

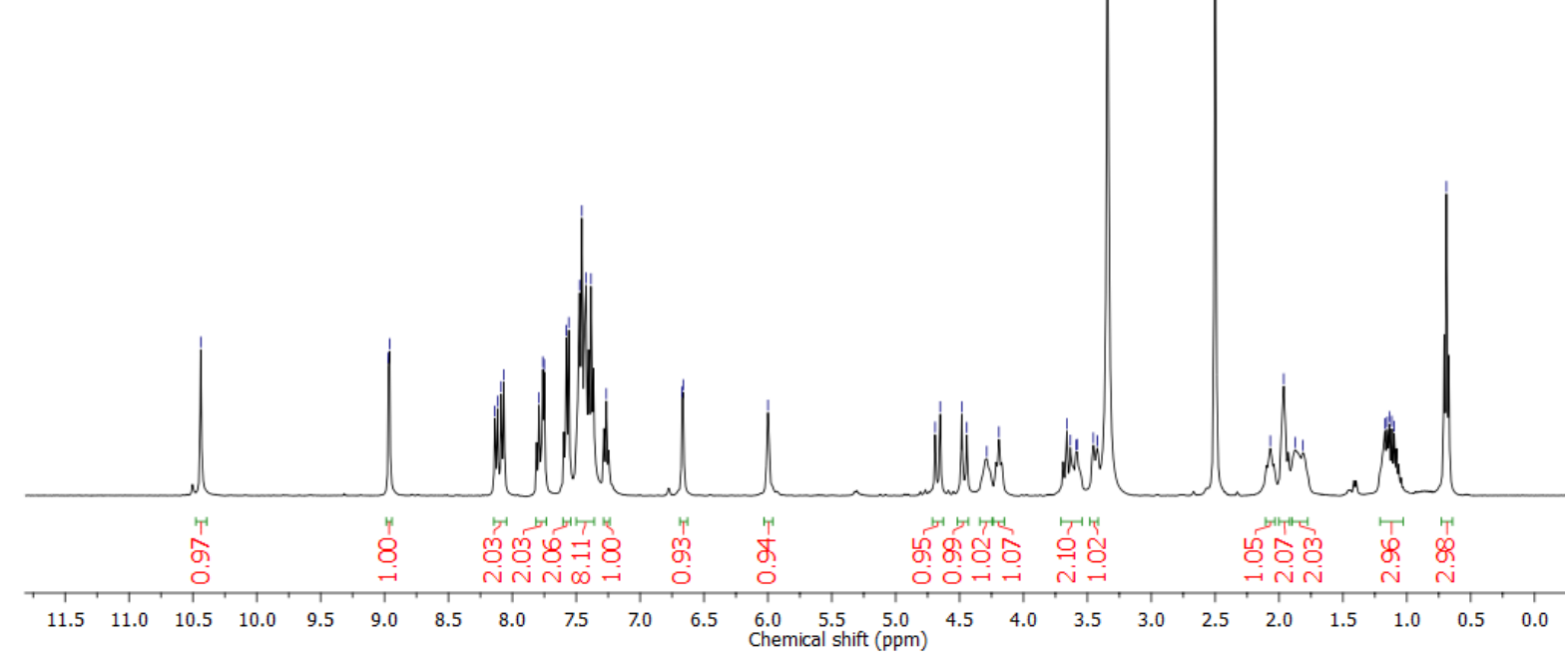

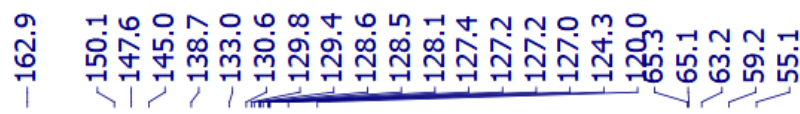

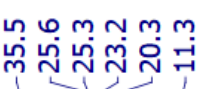

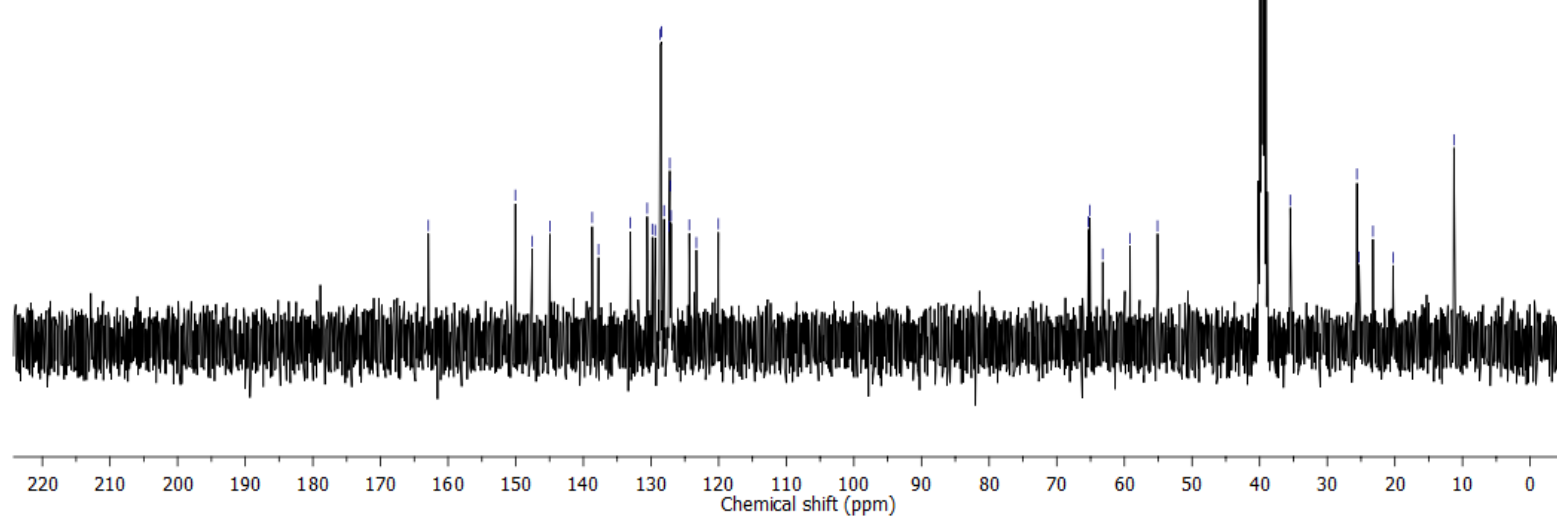

Fig. S27 ${ }^{1} \mathrm{H}$ NMR (400 MHz) and ${ }^{13} \mathrm{C}$ NMR (100 MHz) spectra of compound 45 in DMSO- $d_{6}$. 

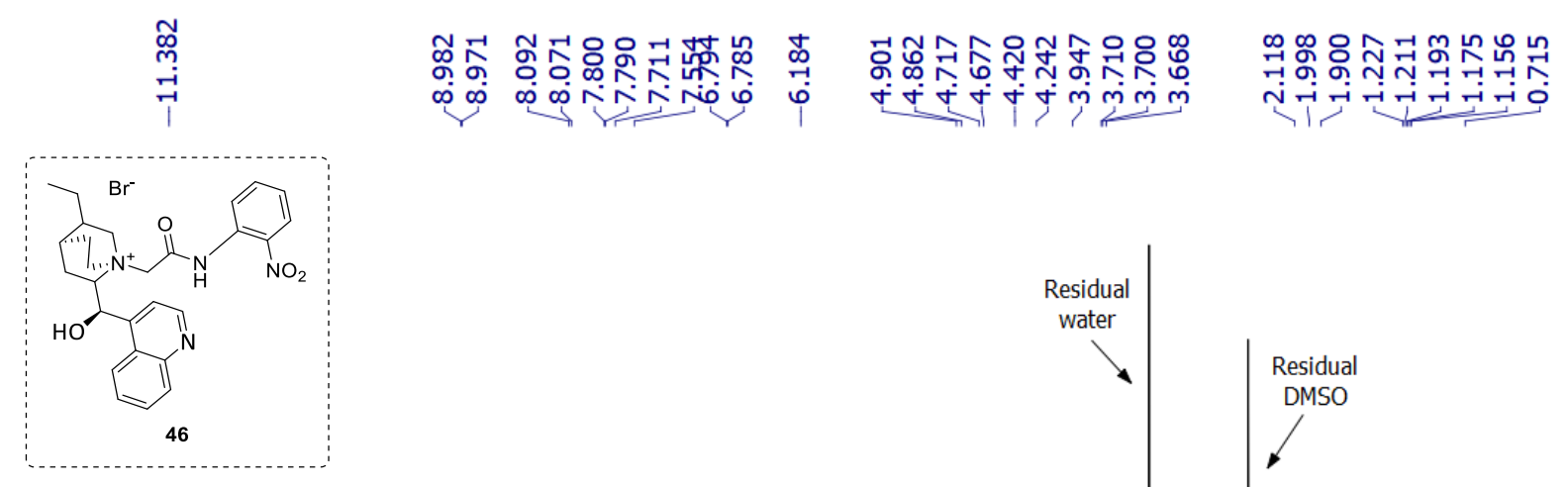

悉

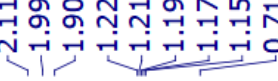

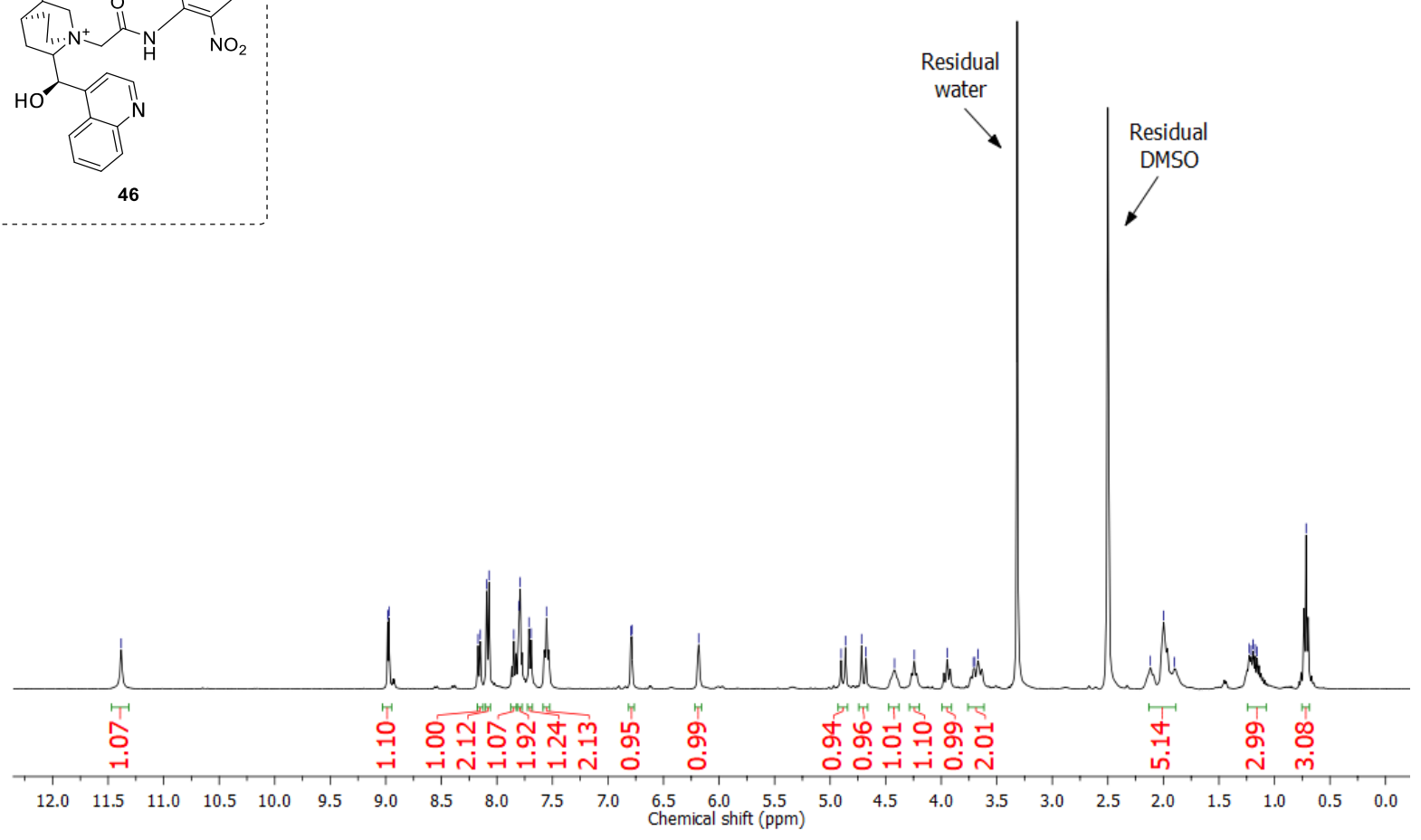

ㄴ.

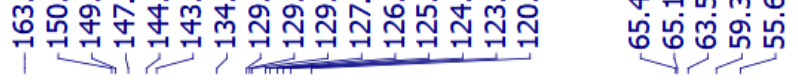

$\ln \pi+a d n$

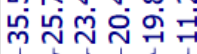

1

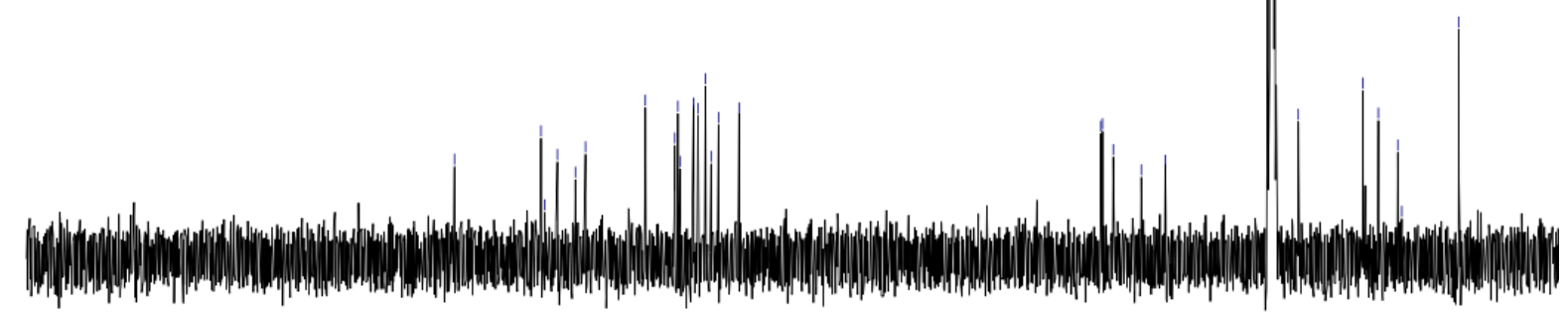

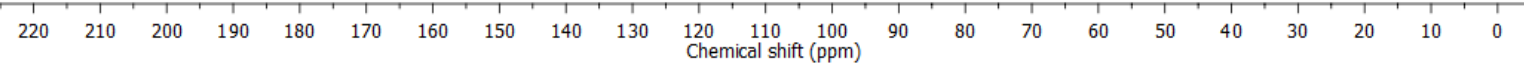

Fig. S28 ${ }^{1} \mathrm{H}$ NMR (400 MHz) and ${ }^{13} \mathrm{C}$ NMR (100 MHz) spectra of compound 46 in DMSO- $d_{6}$. 


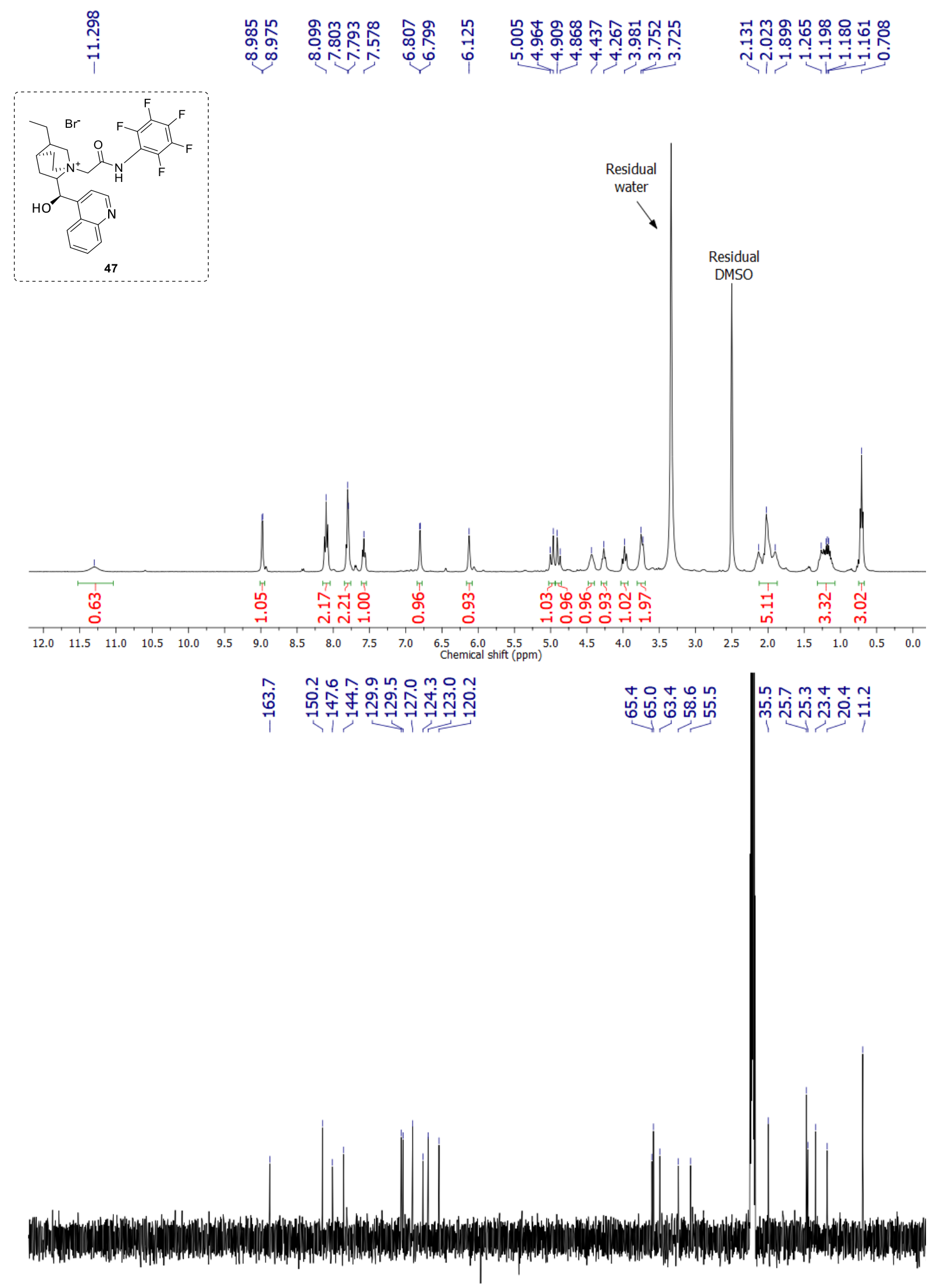

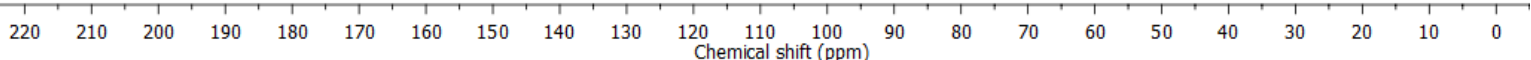

Fig. S29 ${ }^{1} \mathrm{H}$ NMR (400 MHz) and ${ }^{13} \mathrm{C}$ NMR (100 MHz) spectra of compound 47 in DMSO- $d_{6}$. 


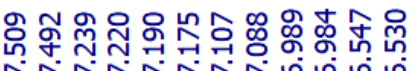

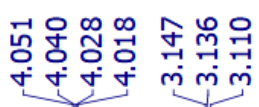
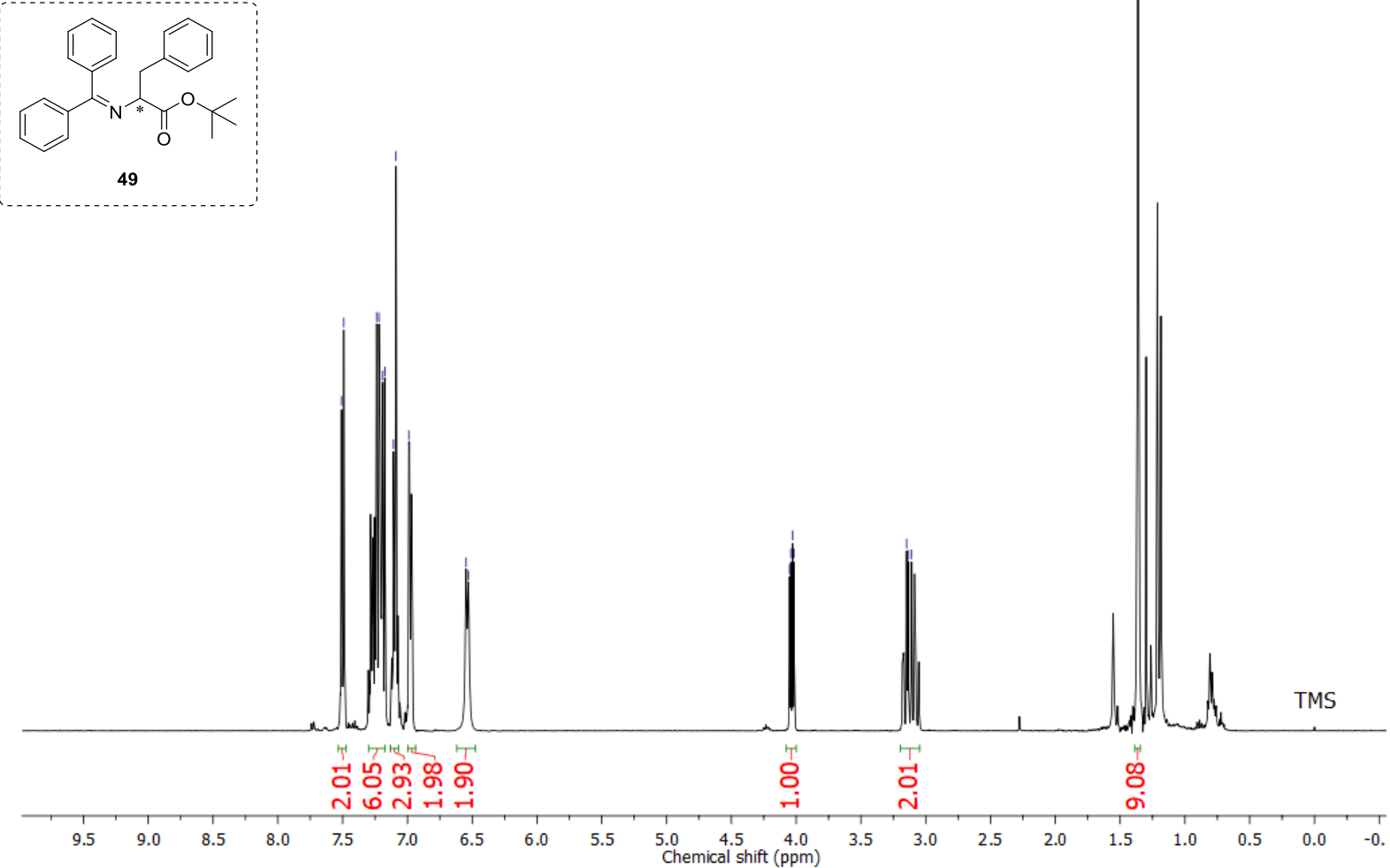

Fig. $530{ }^{1} \mathrm{H}$ NMR (400 MHz) spectrum of compound 49 in $\mathrm{CDCl}_{3}$.

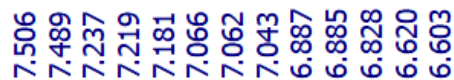

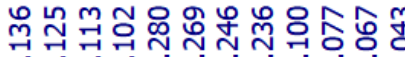
ナசரச

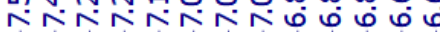
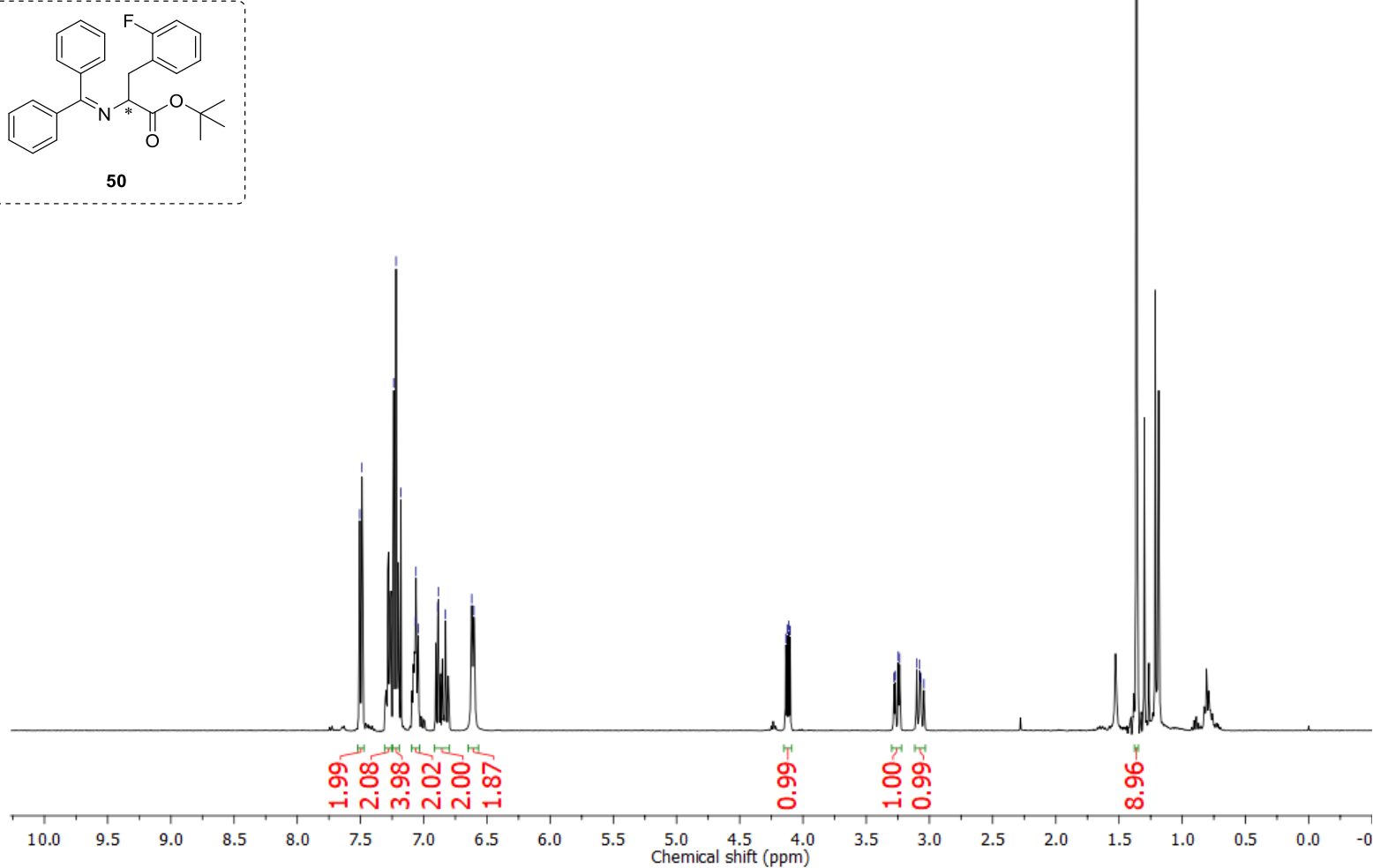

Fig. $\mathbf{S 3 1}{ }^{1} \mathrm{H}$ NMR (400 MHz) spectrum of compound $\mathbf{5 0}$ in $\mathrm{CDCl}_{3}$. 


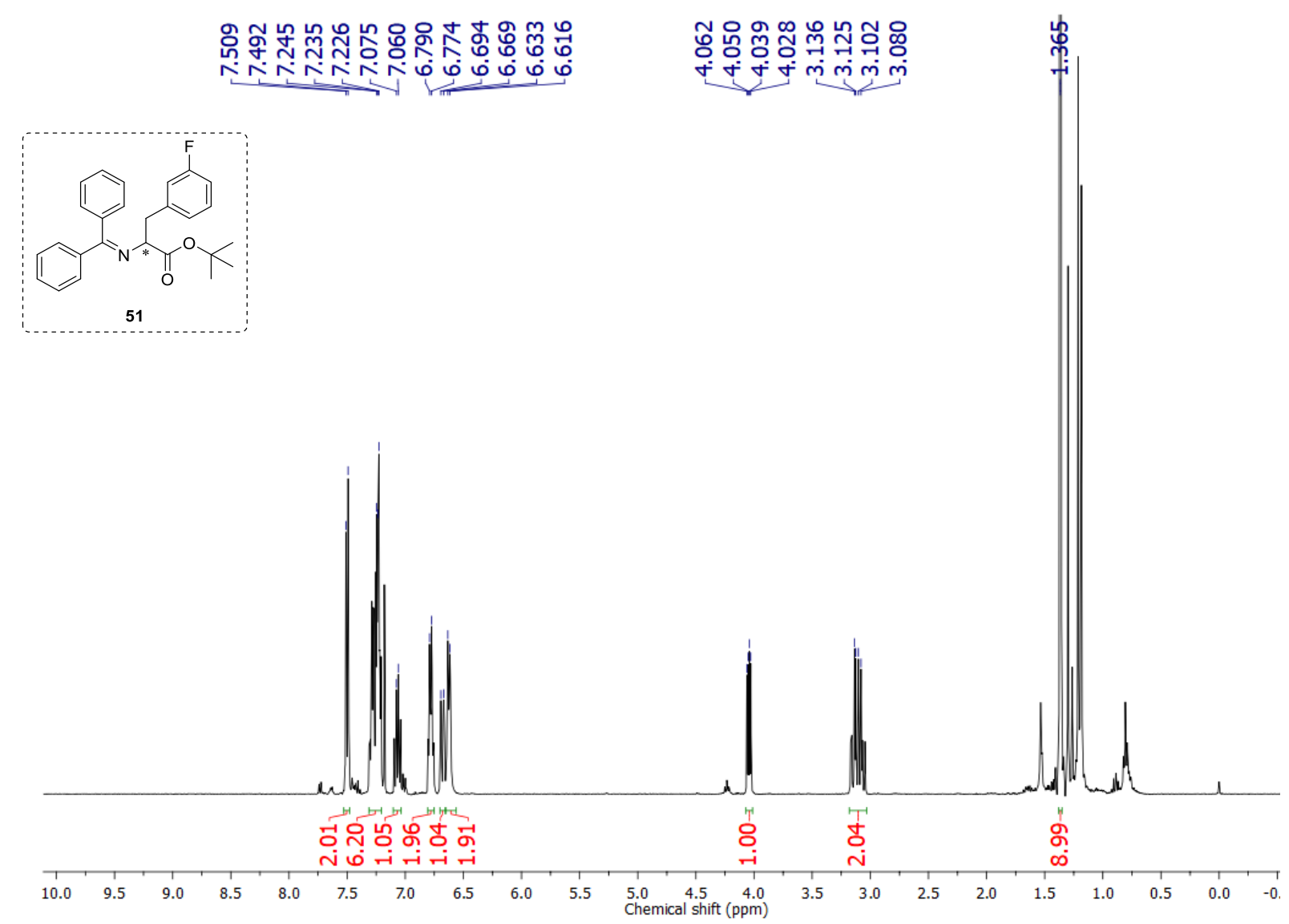

Fig. $\mathbf{S 3 2}{ }^{1} \mathrm{H}$ NMR (400 MHz) spectrum of compound $\mathbf{5 1}$ in $\mathrm{CDCl}_{3}$.

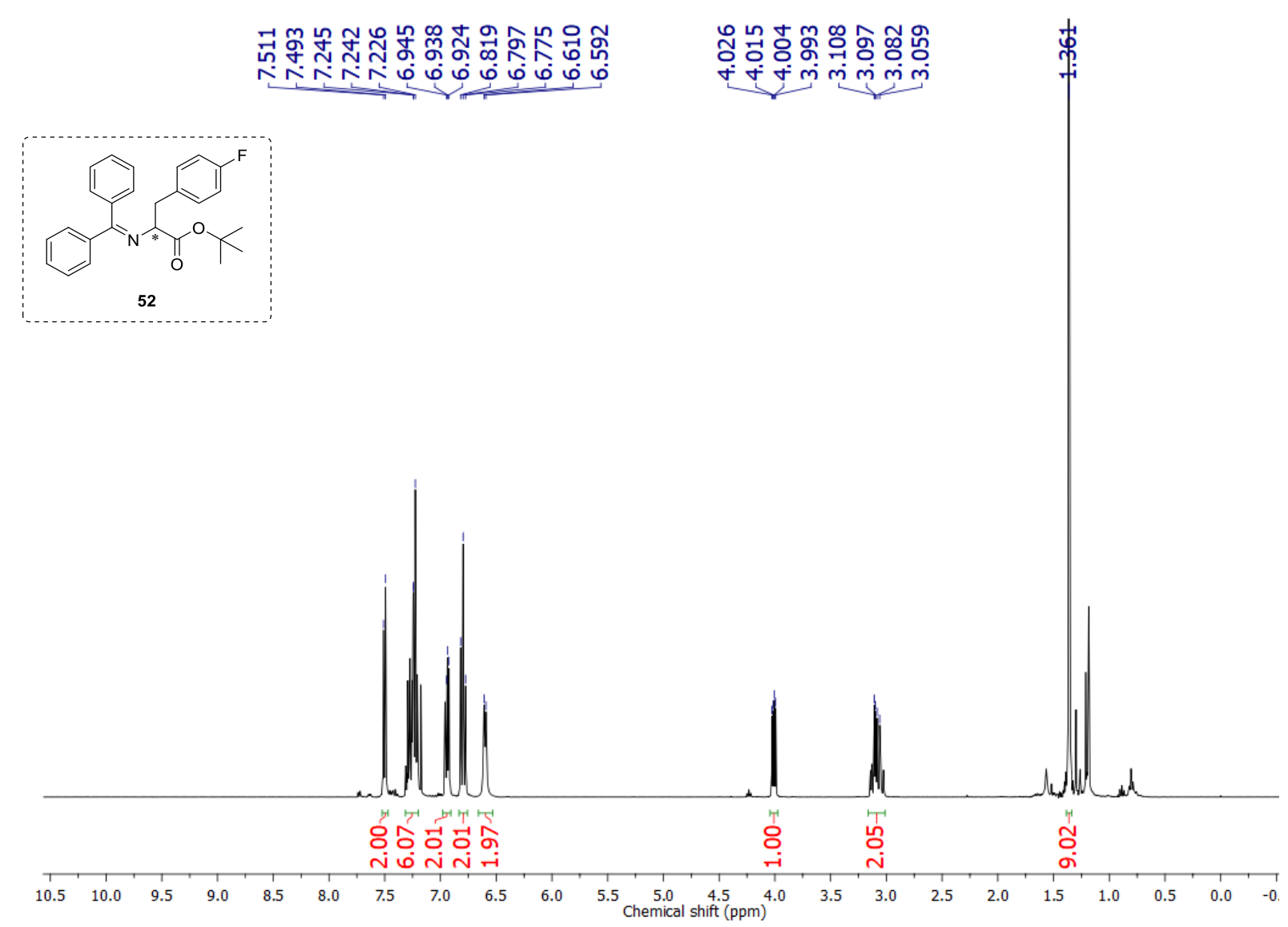

Fig. $\mathbf{S 3 3}{ }^{1} \mathrm{H}$ NMR (400 MHz) spectrum of compound 52 in $\mathrm{CDCl}_{3}$. 


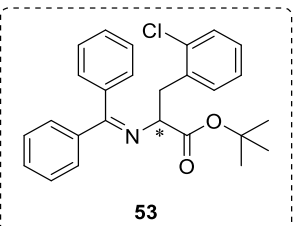

53

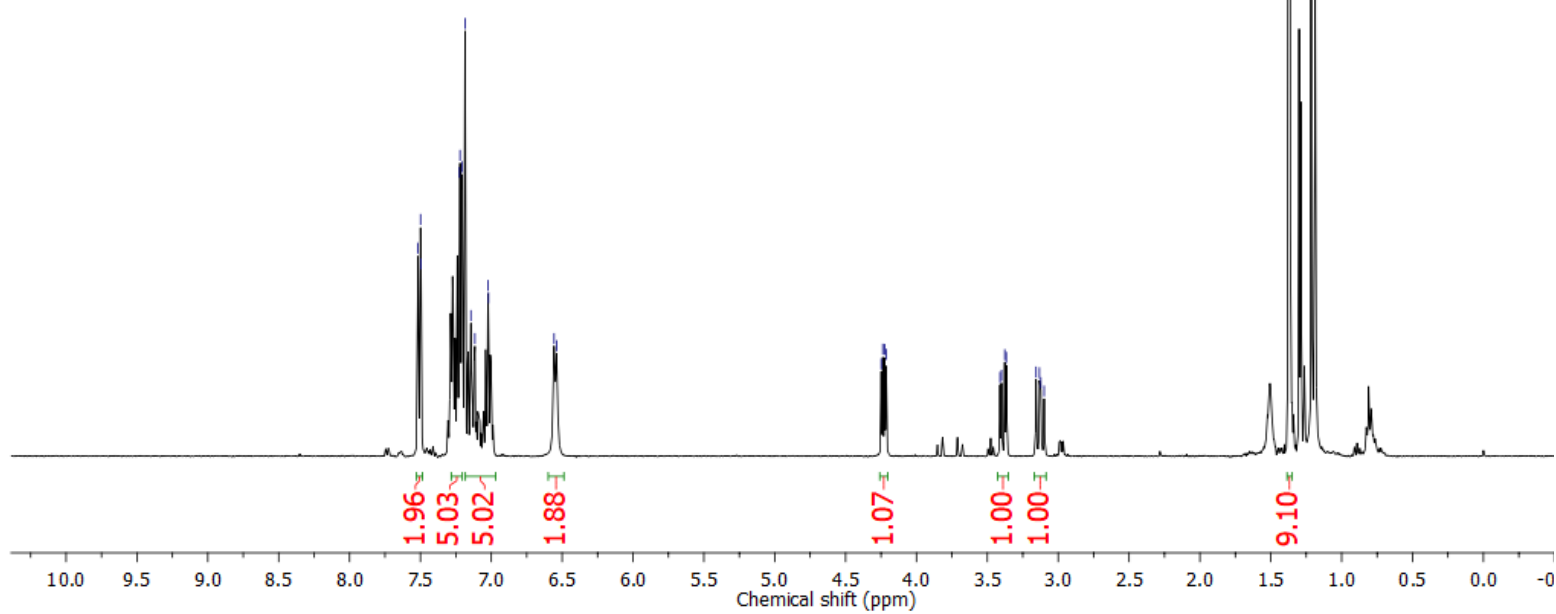

Fig. S34 ${ }^{1} \mathrm{H}$ NMR (400 MHz) spectrum of compound 53 in $\mathrm{CDCl}_{3}$.

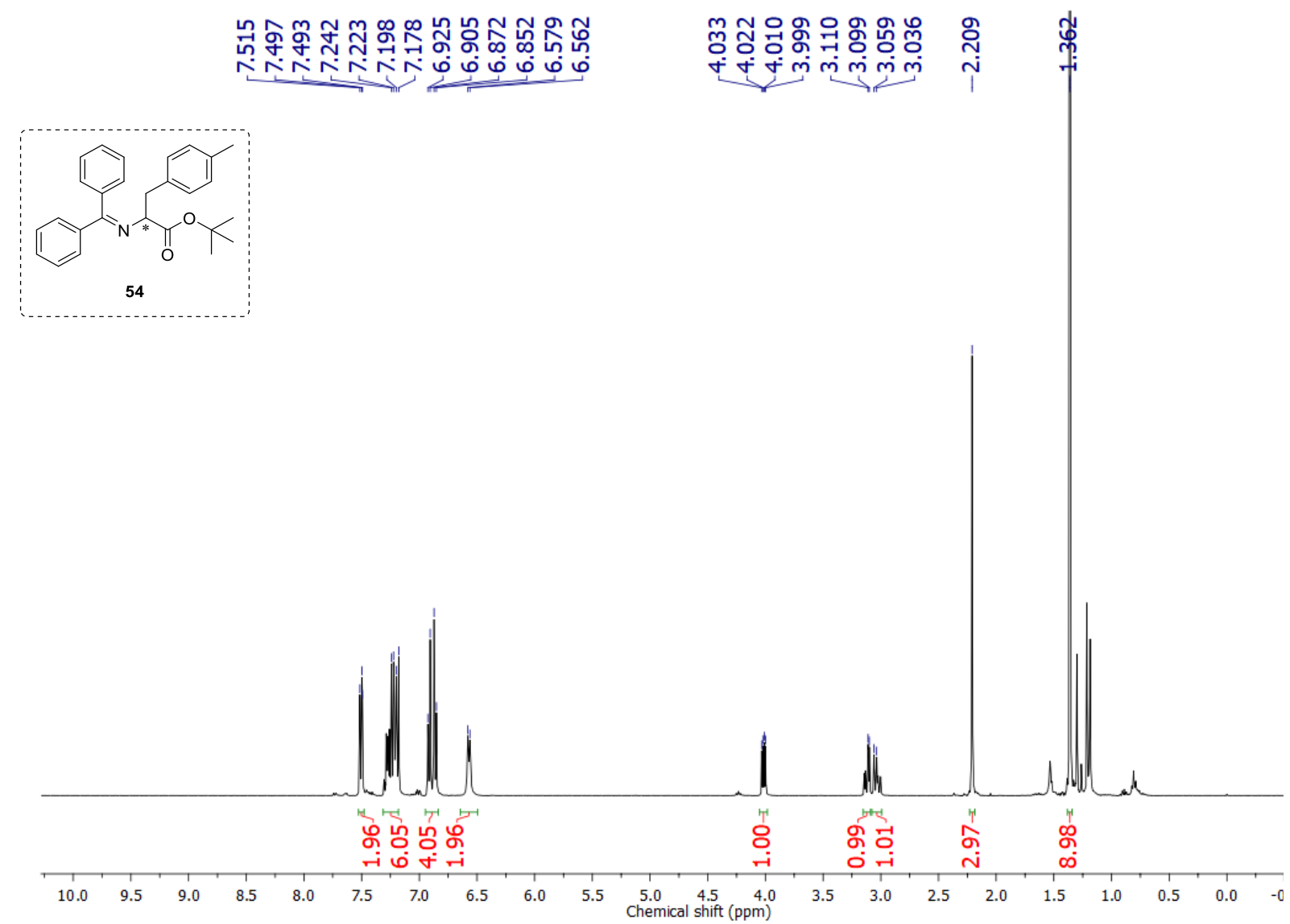

Fig. $\mathbf{S 3 5}{ }^{1} \mathrm{H}$ NMR (400 MHz) spectrum of compound 54 in $\mathrm{CDCl}_{3}$. 


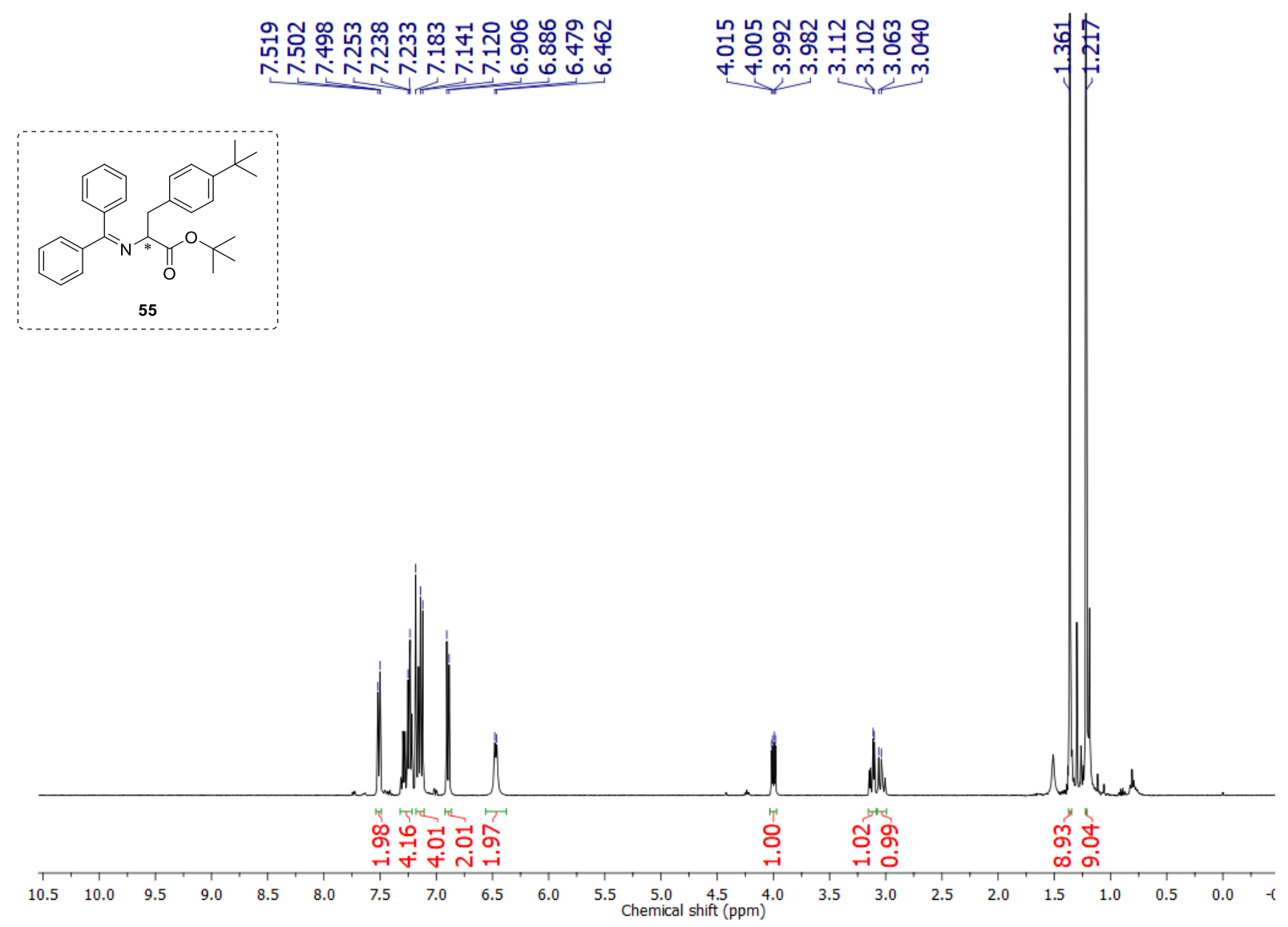

Fig. $\mathbf{5 3 6}{ }^{1} \mathrm{H}$ NMR (400 MHz) spectrum of compound 55 in $\mathrm{CDCl}_{3}$. 


\section{Copies of HPLC chromatograms}

\section{Report}

Sample Name: Ph imine

Method:0.5\% iPrOH/Hex

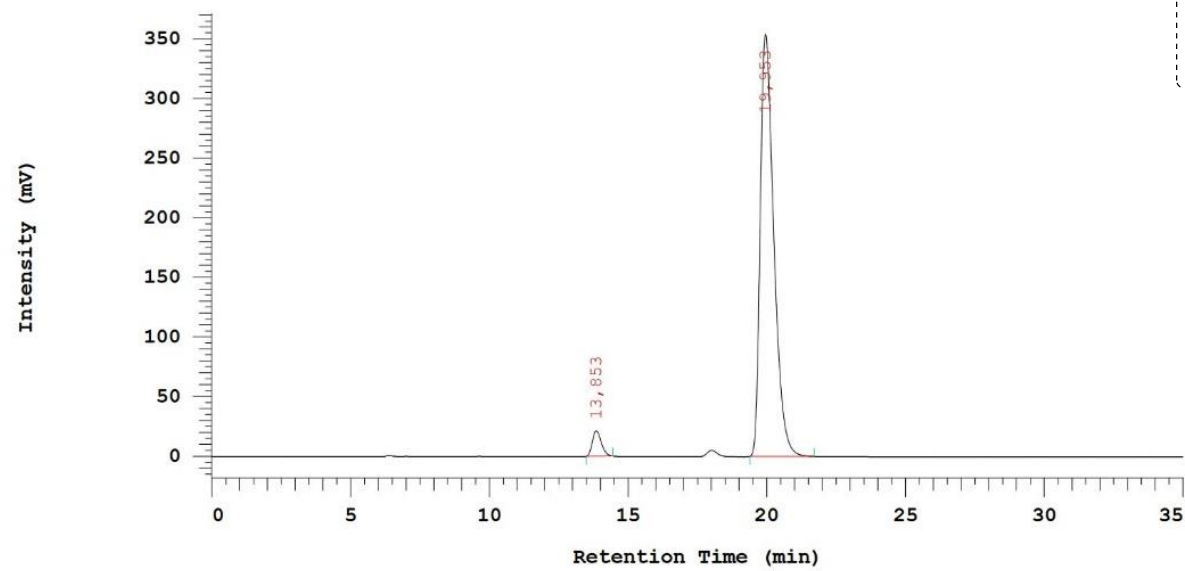

\begin{tabular}{|c|c|c|c|}
\hline No. & RT & Area & Area \% \\
\hline 1 & 13,853 & 472507 & 3,816 \\
\hline 2 & 19,953 & 11909041 & 96,184 \\
\hline
\end{tabular}

Fig. S37 Chromatogram of product 49

\section{Report}

Sample Name: 2F-Ph imine

Method:0.5\% iPrOH/Hex

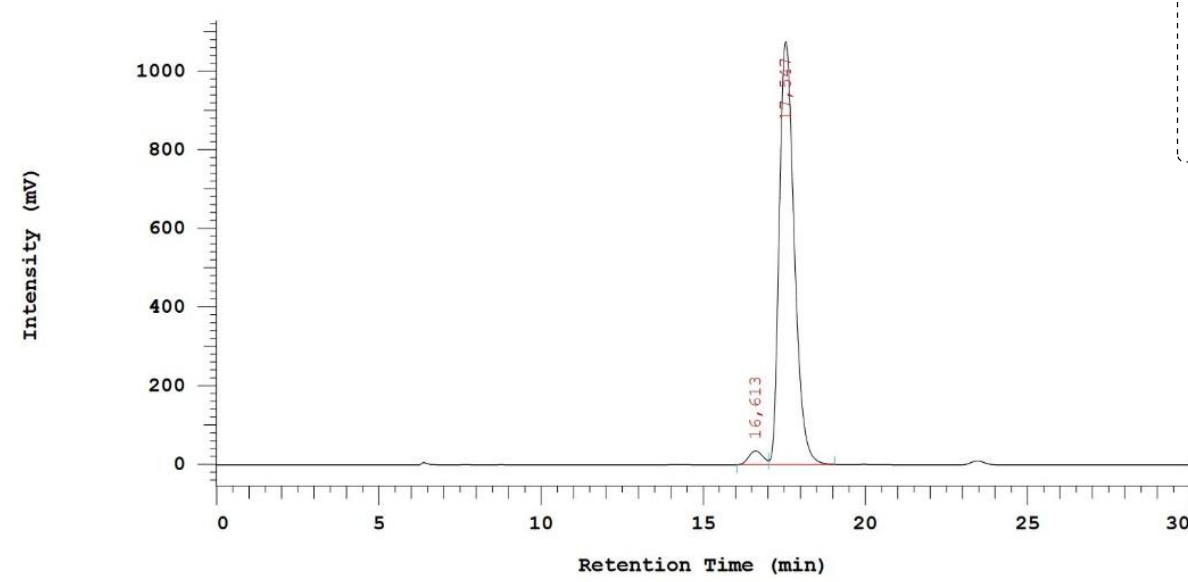

\begin{tabular}{rrrr} 
No. & RT & Area & Area \% \\
\hline 1 & 16,613 & 1055094 & 2,978 \\
2 & 17,547 & 34380034 & 97,022 \\
\hline & & 35435128 & 100,000
\end{tabular}

Fig. $\mathbf{S 3 8}$ Chromatogram of product $\mathbf{5 0}$ 


\section{Report}

Sample Name: 3F-Ph imine

Method:0.5\% iPrOH/Hex

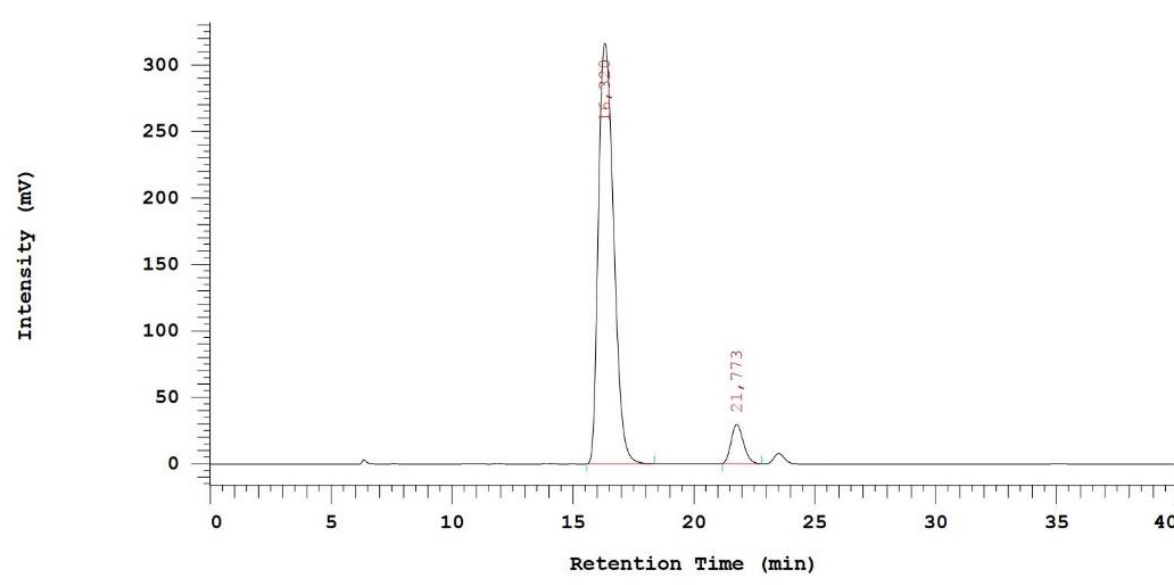

\begin{tabular}{|c|c|c|c|}
\hline No. & RT & Area & Area \% \\
\hline 1 & 16,320 & 13768468 & 93,000 \\
\hline 2 & 21,773 & 1036365 & 7,000 \\
\hline & & 14804833 & 100,000 \\
\hline
\end{tabular}

Fig. S39 Chromatogram of product $\mathbf{5 1}$

\section{Report}

Sample Name: 4F-Ph imine

Method: $0.5 \%$ iPrOH/Hex

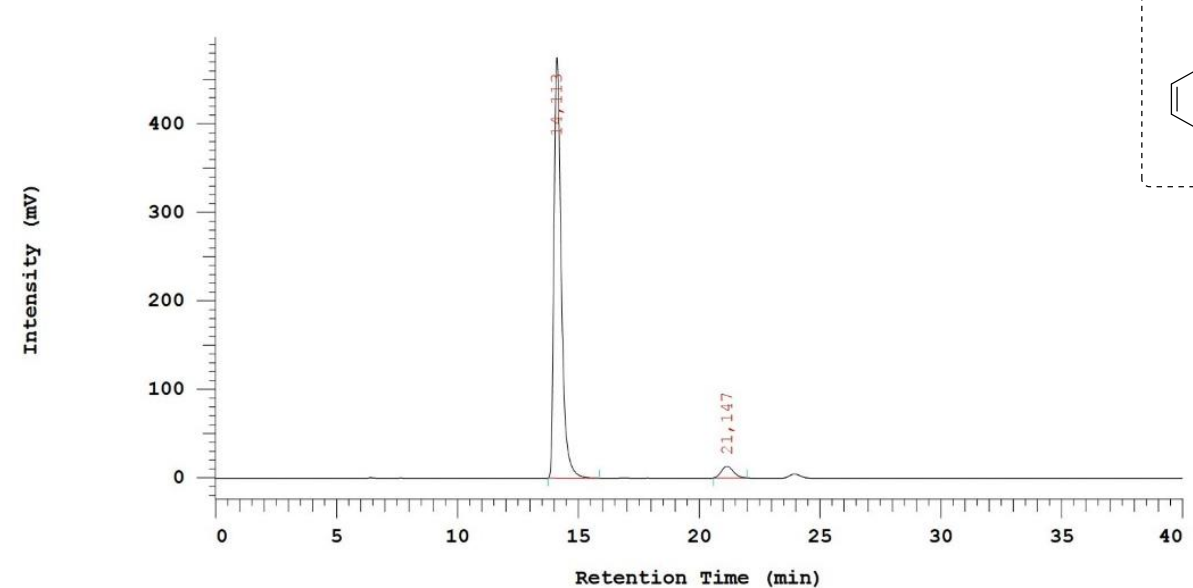

\begin{tabular}{rrrr} 
No. & RT & Area & \multicolumn{1}{c}{ Area \% } \\
\hline 1 & 14,113 & 9843031 & 95,645 \\
2 & 21,147 & 448150 & 4,355 \\
\hline & & 10291181 & 100,000
\end{tabular}

Fig. $\mathbf{5 4 0}$ Chromatogram of product $\mathbf{5 2}$ 


\section{Report}

Sample Name: 2Cl-Ph imine

Method:0.5\% iPrOH/Hex

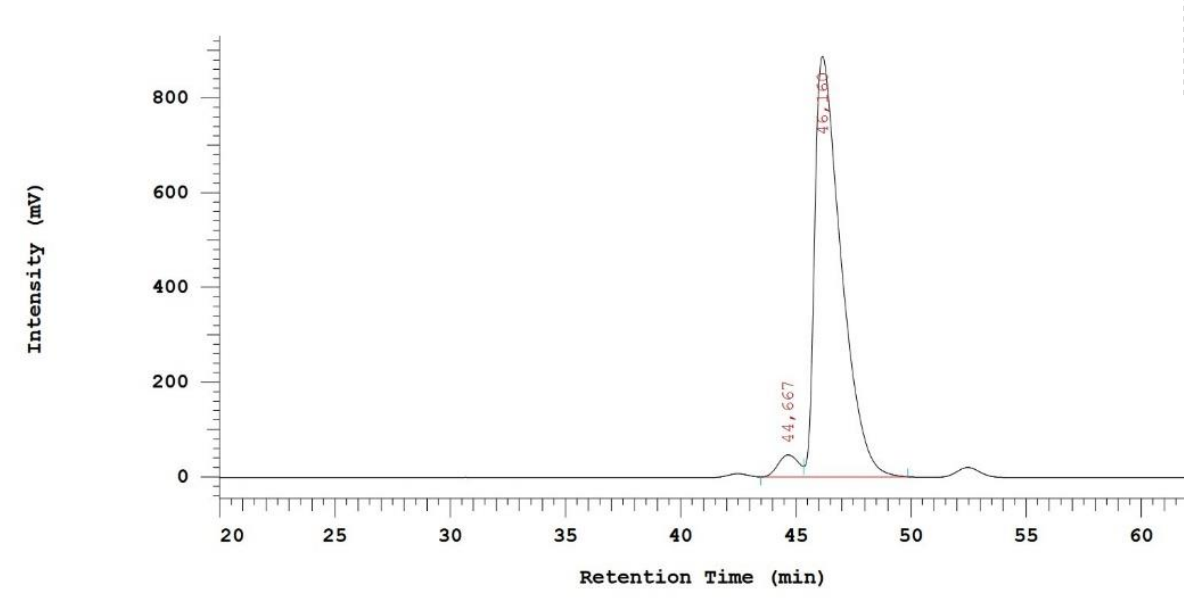

\begin{tabular}{rrrr} 
No. & RT & Area & \multicolumn{1}{c}{ Area \% } \\
\hline 1 & 44,667 & 2805347 & 3,831 \\
2 & 46,160 & 70423168 & 96,169 \\
\hline & & 73228515 & 100,000
\end{tabular}

Fig. S41 Chromatogram of product $\mathbf{5 3}$

\section{Report}

Sample Name: 4Me-Ph imine

Method:0.5\% iPrOH/Hex

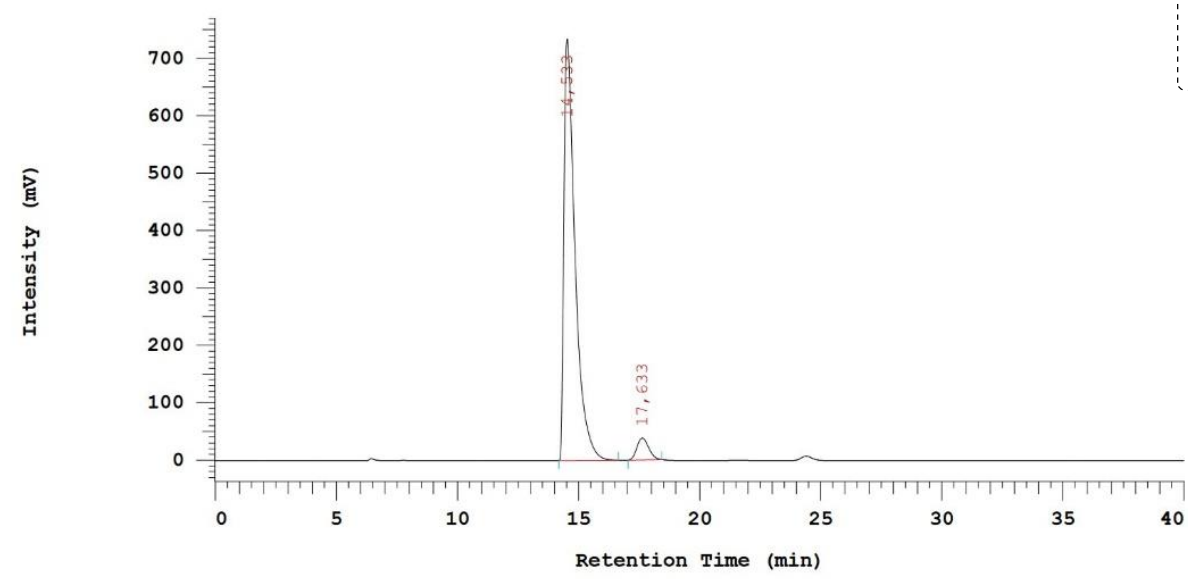

\begin{tabular}{|c|c|c|c|}
\hline No. & RT & Area & Area \% \\
\hline 1 & 14,533 & 23201233 & 94,772 \\
\hline 2 & 17,633 & 1279956 & 5,228 \\
\hline
\end{tabular}

Fig. S42 Chromatogram of product 54 


\section{Report}

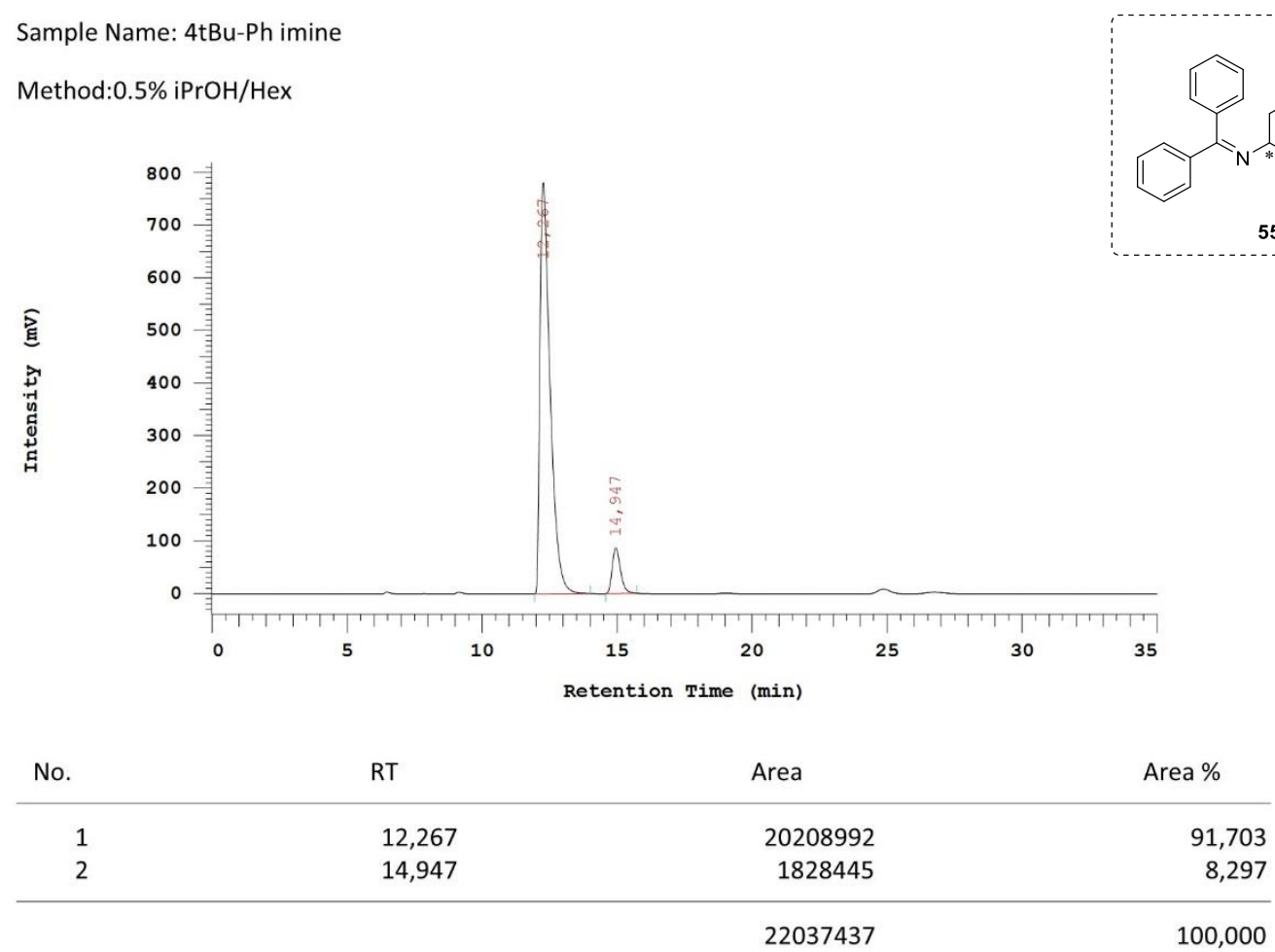

Fig. $\mathbf{5 4 3}$ Chromatogram of product $\mathbf{5 5}$

\section{References}

[1] Agilent (2011). CrysAlis PRO. Agilent Technologies, Yarnton, England.

[2] Sheldrick, G. M. Acta Cryst., 2008, A64, 112-122.

[3] Feng, D., Xu, J., Wan, J., Xie, B., Ma, X., Catal. Sci. Technol., 2015, 5, 2141-2148.

[4] Noisier, A.F.M., Harris, C.S., Brimble, M.A., Chem. Commun., 2013, 49, 7744-7746

[5] Wang, X., Yin, L., Yang, T., Wang, Y., Tetrahedron: Asymmetry, 2008, 18, 108-114.

[6] Waser, M., Gratzer, K., Herchla, R., Müllera, N., Org. Biomol. Chem., 2012, 10, 251-254. 